\title{
Staff-Child Interaction Therapy versus Treatment as Usual in Community-Based Wraparound Services: An Evaluation of Feasibility and Child Behavior Problems
}

Nancy M. Wallace

Follow this and additional works at: https://researchrepository.wvu.edu/etd

\footnotetext{
Recommended Citation

Wallace, Nancy M., "Staff-Child Interaction Therapy versus Treatment as Usual in Community-Based Wraparound Services: An Evaluation of Feasibility and Child Behavior Problems" (2017). Graduate Theses, Dissertations, and Problem Reports. 6892.

https://researchrepository.wvu.edu/etd/6892

This Dissertation is protected by copyright and/or related rights. It has been brought to you by the The Research Repository @ WVU with permission from the rights-holder(s). You are free to use this Dissertation in any way that is permitted by the copyright and related rights legislation that applies to your use. For other uses you must obtain permission from the rights-holder(s) directly, unless additional rights are indicated by a Creative Commons license in the record and/ or on the work itself. This Dissertation has been accepted for inclusion in WVU Graduate Theses, Dissertations, and Problem Reports collection by an authorized administrator of The Research Repository @ WVU. For more information, please contact researchrepository@mail.wvu.edu.
} 
Staff-Child Interaction Therapy versus Treatment as Usual in Community-Based Wraparound Services: An Evaluation of Feasibility and Child Behavior Problems

Nancy M. Wallace, MS

Dissertation submitted to the

Eberly College of Arts and Sciences at West Virginia University

in partial fulfillment of the requirements for the degree of

Doctor of Philosophy in Psychology

Cheryl B McNeil, Ph. D., Chair

Christina L. Duncan, Ph. D.

Elisa Krackow, Ph. D.

Melissa Blank, Ph. D.

Ann Richards, Ph.D.

Department of Psychology

Morgantown, West Virginia

2016

Keywords: Parent-Child Interaction Therapy, Wraparound, Dissemination, Disruptive Behavior Copyright 2016 Nancy M. Wallace 


\begin{abstract}
Staff-Child Interaction Therapy versus Treatment as Usual in Community-Based Wraparound Services: An Evaluation of Feasibility and Child Behavior Problems
\end{abstract}

\title{
Nancy M. Wallace
}

Wraparound services are a well-known, widely implemented community-based model developed to treat children with a variety of severe emotional disturbances (Clark \& Clarke, 1996). Although results have demonstrated some positive outcomes (Suter \& Bruns, 2009), significant weaknesses have also been noted including negative findings (Bertram, Suter, Bruns \& O'Rourke, 2011) and a variety of methodological limitations (Suter \& Bruns, 2009). States have recently begun to examine the empirical basis of wraparound programs to better understand their implementation and effectiveness (Community Data Roundtable, 2015).

The current study examined the implementation of Staff-Child Interaction Therapy (SCIT), a manualized treatment developed at West Virginia University to treat children between ages 2-9 years with severe behavior problems. Based off of Parent-Child Interaction Therapy (PCIT; McNeil \& Hembree-Kigin, 2010), an established, evidence-based practice for young children with disruptive behavior disorders, SCIT was implemented by bachelors-level, community-based wraparound therapists during in-home treatment sessions with their child clients and their primary caregivers. Therapists (SCIT: $n=41$; TAU: $n=32$ ) were primarily Caucasian $(87.7 \%)$ females $(84.9 \%)$ with 45.15 months of therapy experience. Child clients were primarily male $(76.1 \%)$ with an average age of 5.44 years. Children were primarily diagnosed with an Autism Spectrum Disorder $(n=44)$, Attention Deficit Hyperactivity Disorder $(n=19)$, Disruptive Behavior Disorder Not Otherwise Specified $(n=18)$, Oppositional Defiant Disorder $(n=16)$ Conduct Disorder $(n=3)$, Post Traumatic Stress Disorder $(n=2)$, Intellectual Disability $(n=2)$, and another diagnosis $(n=18)$. Many children possessed multiple diagnoses.

SCIT staff were trained in a series of three workshops, spaced approximately seven weeks apart, in which the Child Directed Interaction (CDI; relationship building) and Adult Directed Interaction (ADI; discipline) phases of treatment were taught. Workshops included didactics, live role play, quizzes and practice toward mastery of CDI and ADI skills.

Implementation of the treatment began following the second workshop. SCIT therapists received consultation calls throughout treatment to promote fidelity. Attention control therapists received three workshops and continued to implement treatment as usual with their clients. Attention control workshops included didactics and discussion of compassion fatigue, vicarious trauma, and workforce turn over. No SCIT skills were taught. Primary caregivers and therapists completed the Eyberg Child Behavior Inventory (ECBI; Eyberg \& Pincus, 1999) prior to, during, and following treatment. Qualitative information was gathered on therapists' perceptions of treatment as usual, workshop satisfaction, and SCIT following treatment.

Primary results indicated that parents believed that the intensity of children's behavior problems decreased significantly more for children in the SCIT condition as compared to children in the TAU condition following SCIT implementation $(F(1,33)=5.135, p=.03)$. Similarly, a trend toward significance indicated that behavior problems decreased more for children in the SCIT condition as compared to children in the attention control TAU condition ( $F$ $(1,37)=3.69, p=.06$ from the therapist's perspective. Large effect sizes for children in the 
SCIT group were present according to parents and therapists as compared to small to medium effect sizes for children in the attention control group. Additionally, SCIT therapists $(n=19)$ delivered an average of 7.82 hours of SCIT and an average of 43.39 hours of TAU service to SCIT clients over the 7 -week course of treatment. TAU therapists $(n=32)$ spent an average of 9.42 hours per week with the client and delivered an average of 65.95 total hours of service over the 7-week course of treatment.

Feasibility analyses indicated that the materials needed for SCIT training would cost approximately $\$ 1,145$ per group of eight therapists ( $\$ 143.13$ per therapist) to implement SCIT over a three-day training. Attrition results indicated that the primary cause of attrition between the SCIT therapists $(20.45 \%)$ and TAU therapists $(21.88 \%)$ was therapist withdrawal from the agency. SCIT therapists indicated significantly higher levels of satisfaction with trainings as compared to attention control therapists $(t(69)=5.98, p<.01)$. Qualitatively, therapists in both groups indicated that workshop structure, workshop material, and instructor qualities were particular strengths of the workshops. Specifically, SCIT therapists noted that the length of workshops, speed of instruction, and communication regarding training content between the research team and the agency. TAU therapists indicated the following themes when discussing treatment as usual: BHRS policies in conflict with expected treatment outcomes and inconsistency across BHRS treatment resulting in high levels of job stress and therapist turnover.

Results indicated that SCIT may be a more effective, cost-effective intervention for children presenting to wraparound services with disruptive behaviors as compared to wraparound services as usual. Additionally, SCIT therapists demonstrated high rates of acceptability as compared to TAU as SCIT therapists reported a highly positive impact of the short-term intervention on children's behavior and therapist's skills.

Limitations of the current study included high levels of attrition, small sample size, a lack of complete randomization, and a variety of policy-level challenges. Additional research is needed to better understand the impact of SCIT as compared to treatment as usual on children in wraparound services. Further work should focus on improving the effectiveness of wraparound by increasing the quality of therapist training, integrating more components of evidence-based intervention into wraparound services, and decreasing therapist turn over. 


\section{Acknowledgements}

I entered the field of psychology in order to pursue the work I love. Although, my passion continues to drive my professional endeavors, the incredible support, mentorship, and encouragement that I have been fortunate enough to receive have guided my transformation from a naïve student into a confident professional. This dissertation represents the culmination of my personal and professional journey to this point in my career and serves as a testament to the many family members, professors, supervisors, colleagues, and friends who have taught, guided, encouraged, and mentored me along the way and to whom I am ultimately grateful.

First, to my mom, who instilled in me a love for helping others, who first taught me how to write, and who has been my backbone through each triumph and tribulation throughout my life. To my dad, and my brother who continue to believe in me, and who inspire me to challenge myself and fulfill my dreams.

Next, to my outstanding mentor, Dr. Cheryl McNeil - never in my wildest dreams could I have imagined that a random email to a professor on the other side of the world, inquiring about a treatment that I had learned about by accident could have landed me as your graduate student three years later. You have led by words and example. You have reinforced my dedication to the power of relationships, guided me to see the big picture through the details, elevated my writing, and taught me the importance of celebrating each accomplishment. Your warm and generous spirit, optimistic outlook, passion for mentoring others, and unbelievable ability to see past barriers and envision the future have and will continue to impact me in ways that I cannot express in words. You have set the foundation for my career and, through your mentorship, enabled me to blossom into the person and professional that I have always hoped to be. 
To my lab mates, Lauren Quetsch, Cree Robinson, Kelsey McCoy, and the many undergraduate students who so generously gave up their Saturdays to run training workshops and without question, dedicated themselves to the tedious and unpredictable tasks of large-scale research. This project would not have been possible without you. To my committee, I am forever grateful for your time, flexibility, and dedication to this work. Your perspective, support, and guidance have strengthened me as a researcher and enabled this evolving project to come to fruition. To the agency that allowed this project to occur- Family Behavioral Resources (FBR) my thanks go out to all of the administrators, therapists, and supervisors for your willingness to step into the unknown in the hopes of improving mental health services for children and families.

And finally, to the formidable mentors who have led me to this point- Dr. Teresa Senn, Dr. Michael Carey, Dr. Jefferson Singer, and Dr. Audrey Zakriski - you first introduced me to the unbelievable power of research to change the world. The skills you have taught me, along with your unwavering support laid the foundation for my graduate career and continue to inspire each step of my path. To my college rowing coach, Eva Kovach - thank you for all you taught me through rowing - balance, perseverance, strength, and the utmost form of collaboration. You have given me the personal tools to succeed and truly "walk with a purpose." Your influence and the athletic experience you provided continue to impact who I am and all that I do. To my friends, near and far - I am so very grateful for you. You are my invisible army. Your support, encouragement, and remarkable ability to make me laugh have provided the strength I need to persevere and the perspective I need to see into the future.

Thank you does not seem enough to capture the resounding impact of these individuals and so many others to whom I dedicate this work. I can only hope that the influence of this project may serve as a sign of my gratitude for years to come. 


\section{Table of Contents}

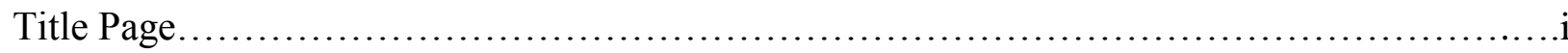

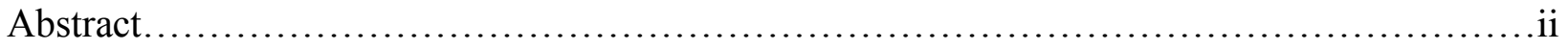

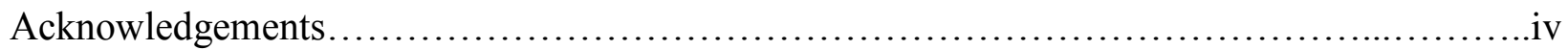

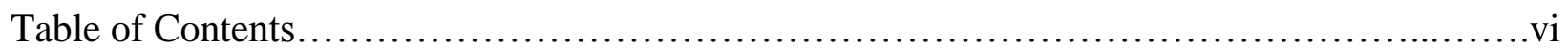

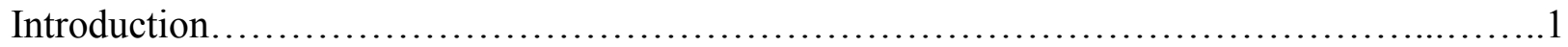

Part I: Wraparound Services.....................................................

General Introduction to Wraparound Services: Definition and History $\ldots \ldots \ldots \ldots \ldots \ldots \ldots . . . .1$

Overview of Population Utilizing Wraparound Services............................ 3

Philosophies and Incidence of Wraparound Services.............................4

Philosophies..........................................................4

Incidence.................................................................

Components of Various Wraparound Programs....................................5

Strengths of Wraparound...................................................

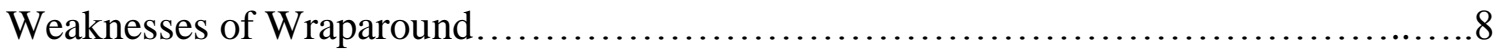

Pennsylvania Wraparound Services - Behavioral Health Rehabilitative Services..........9

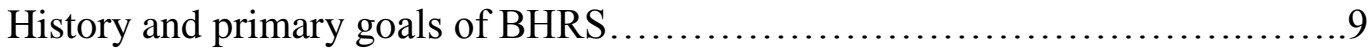

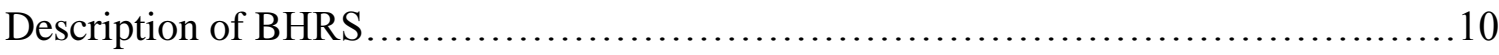

BHRS Policy: Key Components of the Model..................................11

Parental Choice of Service.............................................11

BHRS Team..................................................... 12

BHRS Empirical Questions..........................................13

Part II: Evidence-Based Treatment....................................................... 
Strengths of Evidence-Based Treatment..................................... 15

Weaknesses of Evidence-Based Treatment........................................ 17

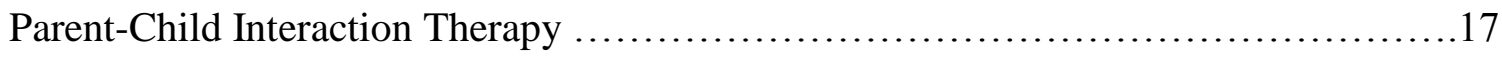

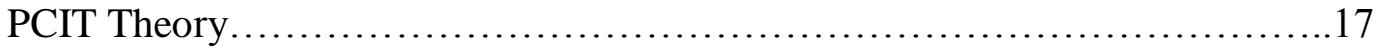

PCIT Treatment................................................... 18

PCIT Research......................................................20

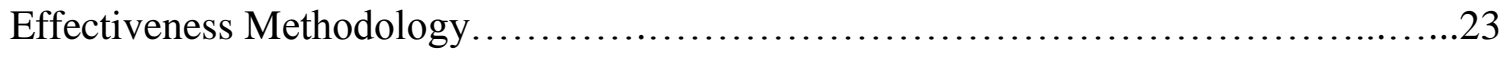

Combining Wraparound and Evidence-Based Approaches............................26

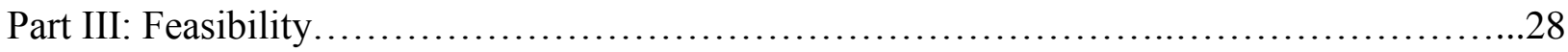

Pilot Study Definition.....................................................28

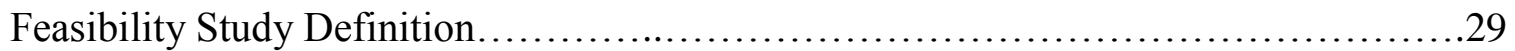

Need for A Feasibility Approach in PCIT Effectiveness Research......................30

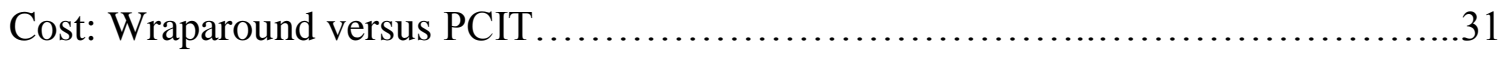

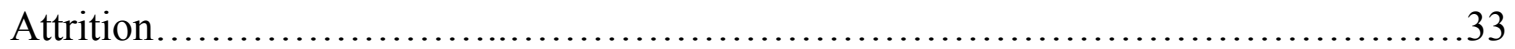

Acceptability........................................................... 34

Sense of Therapist Competence.........................................35

The Current Study: Staff Child Interaction Therapy..............................37

Overview and History of SCIT ....................................... 37

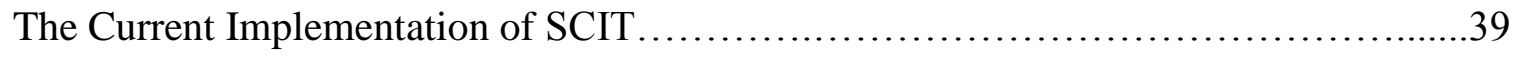

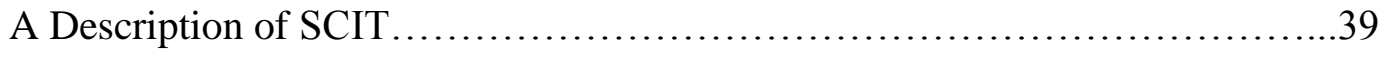

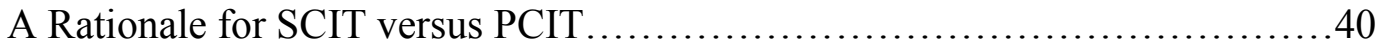

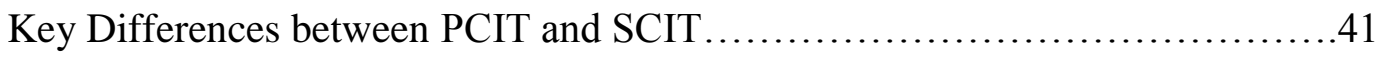

Purpose of the Present Study..............................................42 
Study Hypotheses.......................................................43

Method.......................................................................45

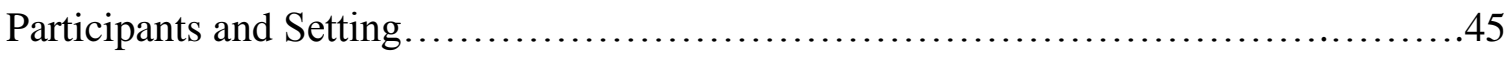

Measures....................................................................... 49

Procedure.............................................................51

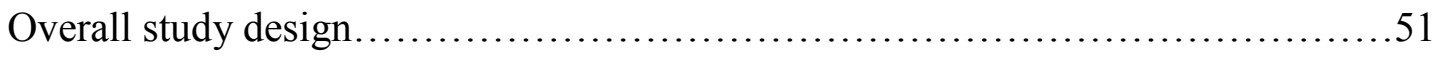

SCIT Day-One Workshop: Therapist Skill Mastery.........................52

Treatment as Usual Day One Workshop .................................54

SCIT Day-Two Workshop: Parent Skill Mastery...........................55

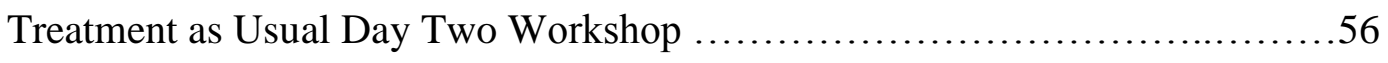

SCIT and Treatment as Usual Post-Treatment Evaluation and Workshop..........56

Consultation Calls.....................................................57

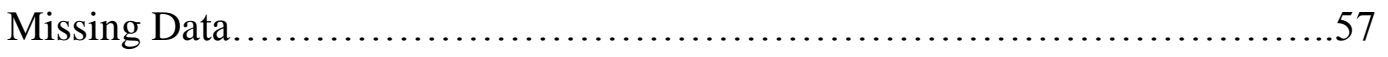

Results......................................................................... 58

Participants........................................................... 58

Therapist Demographic Information.................................58

Child Client Demographic Information..............................6 61

SCIT Fidelity and Dose.........................................62

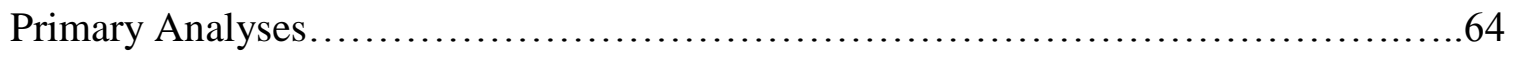

ECBI Parent.....................................................64

ECBI Therapist.................................................66

Comparison of Parent and Therapist ECBI Difference Scores..................68

Feasibility Questions....................................................69 


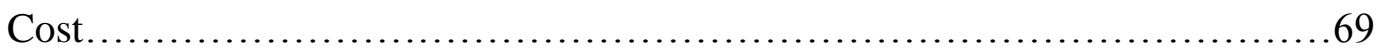

Attrition Status.........................................................69

Therapist Workshop Feedback....................................... 71

SCIT Workshop Feedback.........................................71

TAU Workshop Feedback.......................................... 72

Therapist Sense of Competence.......................................73

Treatment As Usual Description........................................ 75

SCIT Implementation Feedback..................................... 77

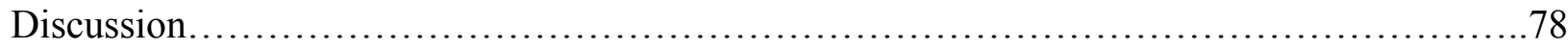

Overview of the Present Study............................................... 78

Child Behavior Problems....................................................... 81

Therapist ECBI................................................ 81

Parent ECBI........................................................ 82

Relationship Between Therapist and Parent Reports........................84

Feasibility Analyses.................................................... 85

Cost of SCIT Materials in Community Implementation......................85

Attrition.................................................................. 86

Therapist Attrition.................................................. 86

Client Attrition.....................................................87

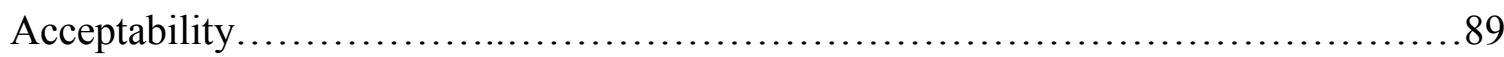

SCIT Workshop Feedback.............................................89

TAU Workshop Feedback ..........................................90

Therapist Sense of Competence.......................................99 
Qualitative Analysis Themes.........................................93

Referral System................................................. 93

Policy-Based Limitations...........................................94

Consumer Model....................................................... 95

Role Expectations................................................95

Parent Involvement and Skill Transfer.................................95

Inconsistent Contextual Factors.......................................96

Lack of Psycho-Education/Specialized Training............................96

Supervision...................................................97

SCIT Treatment Feedback..............................................98

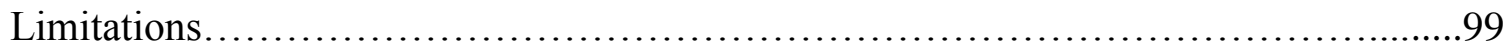

Agency Preparation................................................ 99

Methodological Limitations..........................................101

Implementation-Based Limitations.................................. 104

Future Directions.......................................................... 105

Future Implementation of the SCIT model..................................106

Summary................................................................. 108

References.............................................................. 109

Tables

Table 1: Study Enrollment, Assignment, and Retention Between Groups...............127

Table 2: Therapist Combined Group Means and Standard Deviations at Day One

Workshop.................................................... 128 
Table 3: Therapist between Group Demographic Variables, Chi-Square, and Independent Samples T-Test Analyses at Workshop One.

Table 4. Child Combined Group Means and Standard Deviations at Day One

Workshop

Table 5. Child between Group Demographic Variables, Chi-Square and Independent

Samples T-Test Analyses at Day One Workshop.

Table 6. Correlational Matrix Comparing Workshop Two to Three, Parent Reported

ECBI Difference Scores to SCIT Therapist Fidelity and Dose Variables....

Table 7. ECBI - Parent Perspective - Workshops One, Two, and Three.

Table 8. ECBI Means, Standard Deviations, $F$ Ratios, and $p$ values Across

Workshops.

Table 9. ECBI Scores - Parent Perspective, Workshops Two to Three.....

Table 10. ECBI Means and Standard Deviations, F Ratios, and $p$ values across

Workshops Two and Three. .136

Table 11. ECBI Scores - Therapist Perspective, Workshops One, Two, and Three......137

Table 12. ECBI Scores - Therapist Perspective, Workshops Two to Three.............138

Table 13. Anticipated Cost of SCIT Training Materials in Community Settings........139

Table 14. SCIT and TAU Therapist and Client Attrition........................... 140

Table 15. SCIT Workshop Qualitative Feedback Response Themes..................144

Table 16. TAU Workshop Qualitative Feedback Response Themes...................145

Table 17. SSOC Means, Standard Deviations, F ratios, and p values across workshops

One, Two, and Three 146

Table 18. TAU Discussion Themes. 147 
Table 19. SCIT Therapist Feedback following SCIT Implementation

Figures

Figure 1. Parent CDI Skills Across SCIT Sessions (Median).

Figure 2. Parent ECBI Change throughout First, Second, and Third Workshops between the $\operatorname{SCIT}(n=8)$ and TAU $(n=14)$ Groups

Figure 3. Parent ECBI Change throughout the Second, and Third Workshops between the SCIT $(n=17)$ and TAU $(n=18)$ Groups

Figure 4. Percentage of Children who Improved, Worsened or Remained the Same Workshop Two and Workshop Three in the SCIT and TAU Groups -Parent Report.

Figure 5. Therapist ECBI Change throughout First, Second, and Third Workshops between the SCIT $(n=16)$ and TAU $(n=21)$ Groups

Figure 6. Therapist ECBI Change throughout the Second and Third Workshops between the SCIT $(n=18)$ and TAU $(n=21)$ Groups

Figure 7. Percentage of Children who Improved, Worsened or Remained the Same Between Day One Workshop and Day Three Workshop in the SCIT and TAU Groups - Therapist Report

Figure 8. Percentage of Children who Improved, Worsened or Remained the Same Workshop Two and Workshop Three in the SCIT and TAU Groups -Therapist Report. 159

Figure 9. Staff Sense of Competence across Workshops 160 Appendices

Appendix A. Outline of a Typical SCIT Session .161 
Appendix B. Requirements for Certification- PCIT versus SCIT

Appendix C. Differences between PCIT and SCIT .............................. 163

Appendix D. Demographic Questionnaires.................................. 165

Appendix E. TSS Workshop Satisfaction Survey................................. 169

Appendix F. SCIT Final Survey.......................................... 170

Appendix G. Staff Sense of Competence Scale................................171

Appendix H. Timeline of SCIT and Treatment as Usual Workshops..................172

Appendix I. SCIT and TAU Detailed Workshop Schedules........................173

Appendix J. Adult Directed Interaction Command and Consequence Procedure..........178

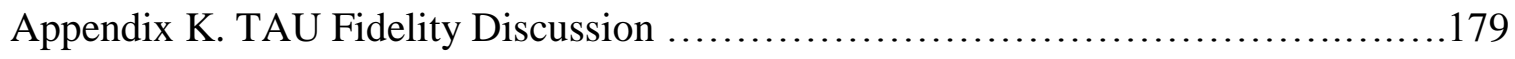

Appendix L. SCIT TSS Consultation Call Outline ................................... 181 


\section{Part I: Wraparound Services}

\section{General Introduction to Wraparound Services: Definition and History}

The inception of the wraparound service model, more broadly known as the "systems of care" approach, dates back to the mid 1970's when movements in Europe and Canada focused on the mobilization of community-based services to curtail the number of individuals diagnosed with complex behavioral and emotional needs entering higher levels of service. Specifically, the development of programs such as the Brownsdale program in Canada, European-based Larch movement, and Kaleidoscope program in Chicago mirrored ongoing efforts to assuage the costly institutionalization of individuals with intellectual disabilities and juvenile offenders while maintaining the safety of community members (VanDenBerg, Bruns, \& Burchard, 2008). Terms such as "least restrictive environment" (Clarke, Schaefer, Burchard, \& Welkowitz, 1992, p. 242) guided primary goals of the intervention in attempts to retain youth in their home and school environments. In the 1980's, Dr. Lenore Behar developed the phrase "wraparound" to contextualize the use of multiple community-based services designed to treat children and youth and prevent induction into institutional care (VanDenBerg, Bruns, \& Burchard, 2008). In this approach, a team of stakeholders is brought together around a child and family often including family members, schools, and other community members invested in the welfare of the child (Walker, Bruns, Conlan, \& LaForce, 2011). The system of care approach aims to form a collaboration between numerous child serving agencies to implement individualized services focused on each family's unique strengths and culture (Bruns, Rast, Peterson, Walker, \& Bosworth, 2006).

The Alaska Youth Initiative (Burchard, Burchard, Sewell, \& VanDenBerg, 1993) developed in 1985 was one of the first organizations to successfully incorporate previously 
institutionalized youth back into community settings. Prior to this time, few treatment options for children with severe needs existed, despite statistics indicating that a significant proportion of children and adults had been diagnosed with a mental health condition that negatively impacted their quality of life (Clark \& Clarke, 1996; Hogan et al., 2003). Following the successful implementation of a wraparound program, the concept was disseminated to over 30 states across the United States. The popularity of such programs throughout the 1980's and 1990's was fueled by state-level and organizational grants awarded by organizations such as the National Institute of Mental Health (NIMH). Leaders in the field gathered at national conferences to share ideas and debate a uniform definition of the widespread but inconsistently operationalized service. As the new decade approached, progress remained slow. The children's mental health system continued to rely on costly hospitalization and residential treatment facilities (New Freedom Commission on Mental Health, 2003 in Bruns, Rast, Peterson, Walker, \& Bosworth, 2006). Significant concerns regarding the overwhelming need for high quality services for youth involved in the juvenile justice system remained (Cocozza \& Skowyra, 2000).

In 2003, the President's New Freedom Commission labeled the dearth of mental health services for youth a public crisis (Hogan et al., 2003). Given the critical need for effective treatment services, a myriad of governmental agencies including the Substance Abuse and Mental Health Services Administration (SAMHSA) and the United States Department of Health and Human services allocated significant funding toward the establishment of community intervention programs throughout the United States to support and treat youth and their families (USDHHS, 2005 as cited in Bruns et al., 2010). Since that time, mental health advocacy for children and families has improved, largely as a result of the establishment of regulations such as the Adoption Assistance and Child Welfare Act (Public Law 96-272), which limited institutional 
placement and returned children to the home environment (Clarke, Schaefer, Burchard, \& Welkowitz, 1992). Additionally, the Federation of Families for Children's Mental Health and regulations such as the Early and Periodic Screening, Diagnosis, and Treatment (EPSDT) in the U.S. Omnibus Reconciliation Act of 1989 (VanDenBerg, Bruns, \& Burchard, 2008). Under such law, Medicaid enables youth to receive diagnostic and treatment services for behavioral health difficulties (VanDenBerg, Bruns, \& Burchard, 2008). Despite well-founded intentions, funding, and availability of services, implementation between states continues to vary, resulting in varying levels of quality and unsystematic program implementation (Clark \& Clarke, 1996).

\section{Overview of Population Utilizing Wraparound Services}

Although wraparound services were primarily designed and continue to be used for youth with severe behavioral and emotional needs, utilization of the service also occurs among children, families, and individuals with varying levels of difficulties (Clark \& Clarke, 1996; (Community Data Roundtable, 2015; Suter \& Bruns, 2009). Often times, children involved in wraparound services are involved in more than one system of care (e.g., child welfare, juvenile justice) (Clark \& Clarke, 1996). Broadly, the diagnostic category of emotional and behavioral disorders (EBD) is often used to encompass the complex needs of children in wraparound. Children may also fall under more than one category of emotional or behavioral impairment including needs in special education and emotional disturbance (Clarke, Schaefer, Burchard, \& Welkowitz, 1992). Although the specific population of interest within wraparound programs varies, children may be diagnosed with Autism Spectrum Disorders, Attention Deficit Hyperactivity Disorder (ADHD), Conduct Disorder (CD), Oppositional Defiant Disorder (ODD), Mood/Anxiety Disorders, Adjustment Disorders, and Psychotic difficulties (Community Data Roundtable, 2015; Wraparound Milwaukee, 2013). In one state-wide implementation of 
wraparound, children had been previously involved in the foster care system, diagnosed with emotional and behavior disturbance, and were not receiving necessary treatment services (Bruns, Rast, Peterson, Walker, \& Bosworth, 2006). Concerns such as aggression, attention difficulties, drug abuse, sexual misconduct, and major illness may be occurring (Wraparound Milwaukee, 2013). Alternative applications of wraparound services have also been successfully used with adult, substance-abusing, criminal offenders to support the diverse needs of this unique population following release (Oser, Knudsen, Staton-Tindall, \& Leukefeld, 2009).

\section{Philosophies and Incidence of Wraparound Services}

Philosophies. From its inception, the wraparound treatment model has aimed to incorporate less restrictive, highly integrated care systems across multiple aspects of a youth's community environment. Theoretically, the approach resembles Bronfenbrenner's (1979) ecological model, which conceptualizes behavior and individual functioning in the context of many layered, interconnected environments and influences (Burchard, Bruns \& Burchard, 2002). It follows then that the model positions treatment directly within a youth's natural environment, namely the home, school and community settings (Burchard, Bruns \& Burchard, 2002). Therefore, this approach aims to assist in enabling the youth's natural environment to promote the generalization and maintenance of treatment gains across time.

The wraparound model is centered on a core set of principles that serve to guide its inception and implementation. Bruns, Walker, \& The National Wraparound Advisory Group (2008) summarized a series of ten tenants that serve as active components of the wraparound approach (see Suter \& Bruns, 2009 for a review). The principles focus on a strengths-based, collaborative, team-oriented approach in which the youth's family, community and individual culture remain an integral component (Copp, Bordnick, Traylor \& Thyer, 2007). Each treatment 
is individualized to the youth and his or her family. Family choice and values are prioritized as integral to treatment success (Bruns, Walker, \& The National Wraparound Advisory Group, 2008; Suter \& Bruns, 2009). Further research has applied such theoretical components to a series of four treatment phases including (1) engaging and preparing the team, (2) developing a plan, (3) implementing a plan, and finally, (4) transitioning away from wraparound services (Suter \& Bruns, 2009; Walker, 2008). Collaboration between all team members and an atmosphere actively promoting respect and dismissing blame is modeled and fostered. Such values attempt to promote shared hopefulness and treatment engagement within families (Winters \& Metz, 2009). Ultimately, if treatment is determined to be unsuccessful, the onus falls upon the plan itself, rather than the family.

Incidence. Throughout the past two decades, research investigating the implementation of the practice has grown. Currently, it is estimated that over 100,000 youth are being served by 1000 programs throughout the United States, Canada, New Zealand, and Europe (Bertram, Suter, Bruns \& O’Rourke, 2011; Suter \& Bruns, 2009; Vandenberg, 2008). This number appears to be growing as more recent estimates have noted that approximately 200,000 children and families were affected by wraparound services (Walker, Bruns, Conlan, \& LaForce, 2011).

\section{Components of Various Wraparound Programs}

It is well known that wide variability between wraparound service lines exist across states and communities. Multiple states including Wisconsin, Vermont, Oklahoma, Mississippi, Massachusetts, and Arizona have instituted wraparound programs. Although wraparound models may include clinical services, the approach differs from pure clinical treatment by coordinating layers of community services across multiple areas of the youth's life (Winters \& Metz, 2009). However, common elements also exist. For example, services are often provided in the home 
environment and may include a diverse arrangement of services including case management, family support, individual and family-based counseling, legal services, respite care, support groups, transportation, tutoring, advocacy, and vocational services (Winters \& Metz, 2009). Therapies such as skills training and work experiences may serve to increase a youth's vocational opportunities and access to a prosocial peer support network (Winters \& Metz, 2009).

Wraparound treatment teams may be made up of a care manager or coordinator along with a family support specialist who facilitates family involvement and advocates for the family throughout the treatment process. The family support specialist plays a unique role in care coordination as he or she often brings experience raising a child with emotional and behavioral difficulties and shares a similar cultural background as family members (Winters \& Metz, 2009). Therefore, this individual brings non-judgmental support and personal experience gaining access to necessary services (Winters \& Metz, 2009).

For example, the wraparound program in Milwaukee has remained focused on youth involved in the Child Welfare and Juvenile Justice systems at imminent risk of residential, hospital, or detention center placement (Winters \& Metz, 2009; Wraparound Milwaukee, 2013). The program has been well recognized for its successful efforts nationwide. Following termination of the grant, the program has sustained and currently serves over 1000 children and families each year (Wraparound Milwaukee, 2013). In addition to coordination of services among over 200 agencies and over 80 services, a mobile crisis intervention team is also available for families to gain immediate access to crisis intervention services (Wraparound Milwaukee, 2013). Services such as crisis stabilization, in-home therapy, transportation support, office-based individual and family therapy, and residential care have been provided (Wraparound Milwaukee, 
2013). Finally, wraparound team members may attend school-based meetings to advocate for the youth and problem solve difficulties with school personnel.

\section{Strengths of Wraparound}

Although results are mixed, promising findings have emerged from implementation and outcome research on wraparound services (Bertram, Suter, Bruns \& O’Rourke, 2011; Painter, 2012). Authors have noted that the treatment potential inherent in the wraparound service model is great (Suter \& Bruns, 2009). As a result, research interest on the effectiveness and outcome of wraparound services has increased (Bertram et al., 2011). In 2004, stakeholders partnered together to create the National Wraparound Initiative (NWI). The group included families, administrators, policy personnel and children dedicated to improving the practice of wraparound. Since then, the group has attempted to unify stakeholders around a common vision for the wraparound system and conducted surveys gauging its impact on the field (Walker, Bruns, Conlan, \& LaForce, 2011).

Some research evidence has resulted in promising outcomes in the wraparound service. Suter and Bruns (2009) conducted the first systematic meta-analysis of seven, controlled outcome studies of wraparound services for youth with serious emotional and behavioral disorders. Results indicated a statistically significant Cohen's D mean effect size of .33 across the seven studies. In other words, the authors noted that a given youth involved in wraparound services had $63 \%$ more successful outcomes than a youth receiving services as usual (Suter \& Bruns, 2009). Specifically, significant outcomes were noted for variables such as mental health outcomes and youth functioning, but effect sizes were small. Another investigation by Hyde, Burchard, and Woodworth (1996) indicated that youth engaging in wraparound services showed better adjustment outcomes. Specifically, a higher percentage of such youth were working or 
attending school, had participated in little harmful behavior, and showed higher levels of community involvement as compared to youth who had not received wraparound services. Additionally, both youth in wraparound services and their parents expressed high levels of satisfaction with such services. Another study of youth in the juvenile justice and child welfare system indicated that youth participating in wraparound services experienced increased clinical functioning, including improved school and behavioral functioning, according to a standardized caregiver interview (Anderson, 2011). Taken together, results indicate that wraparound services have demonstrated some successful outcomes across diverse settings and youth with a variety of emotional and behavioral difficulties.

\section{Weaknesses of Wraparound}

A primary weakness of the wraparound model is a serious lack of uniformity in program operationalization and implementation, thereby producing programs of varying levels of quality (Walker, Bruns, Conlan, \& LaForce, 2011). Some have argued that such divergent implementation is due to the lack of a uniform governing body. Instead, program development and implementation is often conducted on a smaller, local level (Walker, Bruns, Conlan, \& LaForce, 2011). Nevertheless, questions continue to arise surrounding the comparison of wraparound programs when various approaches demonstrate differences in the implementation of core wraparound values (Bruns, Burchard, Suter, Leverentz-Brady, \& Force, 2004; Walker, Bruns, Conlan, \& LaForce, 2011). Additionally, the population served by wraparound presents with a diverse range of needs and mental health conditions, creating complex variability and difficulties for measurement of treatment within the population (Clark \& Clarke, 1996; Suter \& Bruns, 2009; VanDenBerg, Bruns, \& Burchard, 2008). As a result of such wide variability, the 
field has suffered from a lack of measures gauging the quality and fidelity of wraparound implementation (Walker, Bruns, Conlan, \& LaForce, 2011).

Wraparound services have also been met with considerable skepticism related to a myriad of neutral, and in some cases, negative findings (Bertram, Suter, Bruns \& O’Rourke, 2011; Stokes, 2014; Suter \& Bruns, 2009). For example, Bickman, Smith, Lambert and Andrade (2003) noted that when compared to treatment as usual, wraparound services did not demonstrate any significant improvements across a variety of life functioning and life satisfaction variables in a group of children of military members.

In 2003, the National Wraparound Initiative was created to more closely examine and develop the wraparound service model (Bertram et al., 2011). Specifically, the group noted a significant dearth of empirical examination of the wraparound model (Bertram et al., 2011). Furthermore, in 2007, the National Wraparound Initiative (NWI) reported a complete lack of a comprehensive review of wraparound implementation in a peer-reviewed journal (Bertram et al., 2011). Although, a systematic meta-analytic review of the existing outcome literature on wraparound services has been conducted since that time, a range of methodological limitations continue to exist (Suter \& Bruns, 2009). Specifically, small sample sizes, high attrition rates, and weak methodological designs have seriously compromised the validity of study results (Copp, Bordnick, Traylor \& Thyer, 2007; Hyde, Burchard \& Woodworth, 1996; Painter, 2012).

\section{Pennsylvania Wraparound Services - Behavioral Health Rehabilitative Services (BHRS)}

History and primary goals of BHRS. The current study focused on one model of wraparound service delivery entitled Behavioral Health Rehabilitative Services (BHRS), based in Pennsylvania. Similar to other wraparound programs, BHRS is provided in the home, school and, community environments. In the school, the service may be used to maintain children in a 
typical education classroom by providing direct assistance to the child (e.g, behavioral modification) or as an advocate within the larger educational setting (e.g., coordination of education services within the school and between environments) (Understanding "Wraparound" Services for Children in HealthChoices, 2011).

Similar to the broader context of wraparound, the primary goal of BHRS is to utilize community based services and prevent children with complex emotional and behavioral difficulties from utilizing higher-level services (Community Data Roundtable, 2015). Similar to other wraparound service models, children utilizing BHRS services are often involved in multiple systems including juvenile justice, child welfare, and substance treatment.

BHRS has been named one of the oldest forms of mental health for children in Pennsylvania in response to the push for wraparound services during the 1980's and 1990's (Community Data Roundtable, 2015). Given that the BHRS model is not empirically supported, program standards are primarily governed by Medicaid-based policies, costing the state of Pennsylvania hundreds of millions of dollars per year (Community Data Roundtable, 2015).

\section{Description of BHRS}

Multiple steps are involved in initiating BHRS services for a child. First, a parent or caregiver must initiate contact with the child's Behavioral Health Managed Care Organization (MCO) to access a psychologist or psychiatrist in his or her insurance network to conduct an initial evaluation of the needs of the child and family (Understanding "Wraparound" Services for Children in HealthChoices, 2011). MCO's are required to provide at least two alternative providers to allow families to play a guiding role in determining the best choice for their family. Additionally, such providers must be located within 60 minutes from families in rural areas or 30 minutes from families in urban areas. Next, a family must schedule and meet with a participating 
psychologist or psychiatrist. During this appointment, caregivers may discuss emotional and behavioral difficulties experienced by their child and review paperwork from additional evaluations, should such information exist. Additionally, caregivers may identify goals for the child and degree of service needed. Following this meeting, the psychologist recommends a dose of service (hours per week), type of service provided (TSS, MT, BSC), location, and the timeframe of such service. Finally, the evaluator must justify the medical necessity of the treatment. Finally, if a recommendation for wraparound services is provided, an Interagency Service Planning Team (ISPT) meeting will be arranged. Ideally, the providing doctor participates in this meeting in addition to the MCO. During the meeting, the recommendation is discussed and input from all parties is obtained. Services begin approximately 60 days from this meeting. Additionally, the original prescription for service lasts between 4-12 months depending upon the child's diagnosis. In order for services to continue past this time frame, a re-evaluation must occur and another ISPT meeting may be scheduled.

\section{BHRS Policy: Key Components of the Model}

Parental choice of service. Nationally, wraparound services are fundamentally based on the notion that a child and his or her family are in a critical position to provide knowledge, insight, and guidance to the wraparound team. Wraparound services view caregivers as experts on their child's disorder and specific needs throughout treatment (Walker, Bruns, Conlan, \& LaForce, 2011). In practice, the youth and his or her family are allowed to guide all decisions surrounding the child's care as their perspective is considered equally as valuable as that of professionals (Winters \& Metz, 2009). Such principles are designed to counter previous treatment efforts in which families were only peripherally involved in treatment (Walker, Bruns, Conlan, \& LaForce, 2011). It is thought that by helping to inform and guide services, the youth 
and his or her family remain naturally engaged as active members of the treatment team. In BHRS, parents are given the initial freedom to choose which provider they would like to receive services from (Recovery Toolkit, 2011; Performance Standards, 2012). Furthermore, a family is entitled to choose the methodology and/or treatment approach used to treat the child's particular needs (Performance Standards, 2012). If a family determines that a given provider is not meeting the needs of the child and family, they possess the freedom to choose a new provider (Performance Standards, 2012). Thereby, such choices mirror nationally prescribed values of wraparound services.

BHRS Team. A typical BHRS team consists of a bachelor's level clinician, otherwise known as a Therapeutic Support Staff (TSS), who primarily works to implement the treatment plan. This staff member is required to possess a two-year college degree, 60 credit hours toward a degree or a 4-year college degree (Recovery Toolkit, 2011). A primary focus of the TSS may be to modify identified negative behaviors by teaching positive, socially appropriate skills as well as interaction and conflict management techniques (Recovery Toolkit, 2011; Understanding "Wraparound" Services for Children in HealthChoices, 2011). Such skills are designed to assist the child in fostering independence from treatment services (Recovery Toolkit, 2011). The TSS may implement such interventions in the home, school, or community settings. Additionally, a Mobile Therapist (MT) may also be present within the team to provide individual therapy to the child. This staff member may utilize therapeutic strategies to help the child understand his or her emotions in the home or community. A Behavioral Specialist Consultant (BSC) may create a treatment plan and supervise the TSS and MT in conducting its implementation. Finally, the child client is referred to as a "consumer" as he or she is thought to be consuming an individualized treatment plan that could be implemented by any therapeutic support staff 
member. Furthermore, such terminology reflects the powerful impact of family choice within the wraparound model.

BHRS Empirical Questions. A recent white paper produced by Community Data Roundtable conducted a small scale evaluation of BHRS in order to question the empirical efficacy of this costly and widely used service (Community Data Roundtable, 2015). Prior to this evaluation, no empirically based studies had been conducted on BHRS despite decades of implementation (Knapp \& Reed, 2010 in Community Data Roundtable, 2015). Broadly, the paper warns that BHRS utilizes multiple intervention styles to treat diverse diagnoses, ethnicities and genders, making evaluation difficult. Furthermore, such interventions may wax and wane over time depending on factors such as treatment acceptability and client progress. Despite regular psychological evaluations of BHRS clients, evaluating psychologists rarely utilize evidence-based approaches when deciding upon BHRS recommendations.

The Child and Adolescent Needs and Strengths (CANs) Assessment was used to evaluate the effectiveness of BHRS for various populations. Developed to inform clinical care decisions for individualized clients in community mental health, the evaluation outlines five categories of needs (i.e., problem presentation, risk behaviors, functioning, child safety, family/caregiver needs and strengths) and one strengths category. Respondents are asked to rate the severity level of each particular need on a four-point scale in the span of the last 30 days. Although the assessment provides a useful profile for community systems to systematically coordinate care efforts, thus far, the measure has only been used in select populations (CANS-MH Manual, 2008).

The evaluation classified an individual severity score (e.g., least, moderate, intensive, highly intensive) for 8,477 children involved in the BHRS services. Such scores were developed 
to simulate the severity scores used in the CANS. BHRS outcomes based on severity scores were measured over time. Results indicated that children classified as entering BHRS with a lower severity score rise to a higher severity level over time. In contrast, children who enter BHRS with a higher severity score drop to a lower score over time, indicating improvement. However, if treatment outcome is measured across time and children are aggregated across severity levels, no change is present (Community Data Roundtable, 2015). Notably, the aforementioned evaluation did not include a comparison group thereby limiting attributions of causality. Issues such as natural regression to the mean may be of concern. The paper concludes by highlighting the potential use of severity categories during mental health assessment of children. Such assessments may serve great importance in informing professionals and families of children at various severity levels of the potential outcome of BHRS treatment for children with varying needs. Taken together, the paper provides the most up to date assessment indicating significant deficits in the current evaluation practices and effectiveness of BHRS as a community-based intervention, as well as and a clear need for further assessment and development of this widespread, costly program (Community Data Roundtable, 2015). 


\section{Part II: Evidence-Based Treatment}

In an effort to increase the quality, cost-effectiveness, and dissemination of empiricallybased, psychological interventions, The APA Presidential Task Force on Evidence-Based Practice was created and defined evidence-based practice in psychology (EBPP) as, "the integration of the best available research with clinical expertise in the context of patient characteristics, culture, and preferences" (APA Presidential Task Force on Evidence-Based Practice, 2006, p. 273). Within the broader context of empirically supported treatment, three divisions of efficacy had been noted in a 1998 report by Chambless et al. with the Task Force on Psychological Inteventions (Chambless et al., 1998). Such areas included 1) well-established treatments, 2) probably efficacious treatments, and 3) experimental treatments. Specifically, in contrast to a probably efficacious treatment, a well-established treatment must demonstrate empirical superiority over a psychological placebo or when contrasted against another treatment by two separate research groups. However, a probably efficacious treatment must demonstrate an empirical advantage over a waitlist or no treatment control group (Ollendick \& King, 2004). Finally, an experimental treatment may include approaches that have not yet been supported by enough scientific evidence to be rendered probably efficacious or well-established (Ollendick \& King, 2004). Although weaknesses continue to exist, it is clear that evidence-based treatments purport a wide variety of methodological and clinical strengths over non-empirically based approaches.

\section{Strengths of Evidence-Based Treatments}

A recent meta-analysis examining the outcomes of a variety of evidence-based treatments indicated a Cohen's D of .54, falling in the medium effect size range (Weisz, Weiss, Han, Granger, \& Morton, 1995). The authors noted particularly strong effects for child behaviorally- 
based treatments (Weisz, Weiss, Han, Granger, \& Morton, 1995). A separate meta-analysis of 32 studies directly compared evidence based treatment to usual care when participants were randomized across conditions. Results found a smaller but notable effect size of .30. However, the authors noted that the Weisz et al., 1995 effect size of .54 was likely influenced by comparisons between evidence-based treatment and no treatment (e.g., waitlist control) whereas the smaller, aforementioned effect size was likely due to a comparison between evidence-based treatment and treatment as usual (Weisz et al., 2006). Specifically, effect sizes of evidence-based treatments (e.g., Parent-Child Interaction Therapy) note consistently larger effect sizes when examined individually outside of the broad meta-analysis (Thomas \& Zimmer-Gembeck, 2007). Eyberg, Nelson and Boggs (2008) conducted a meta-analytic review of 12 studies of well-established and probably efficacious treatments for children with disruptive behavior problems. Specifically, Incredible Years Child Training (Webster-Stratton \& Hammond, 1997), Multidimensional Treatment Foster Care (Chamberlain \& Reid, 1998), Multi-Systemic Therapy (MST: Henggeler, Meton \& Smith, 1992), Parent-Child Interaction Therapy (PCIT; Schuhmann, Foote, Eyberg, Boggs \& Algina, 1998), Problem Solving Skills Training (PSST; Kazdin, Bass, Siegel \& Thomas, 1989), and Triple P Enhanced (Sanders, Markie-Dadds, Tully \& Bor, 2000) were examined. Each indicated an $80 \%$ or higher proportion of studies with post-treatment, between-group effect sizes of greater than or equal to .20. Furthermore, Silverman et al. (2008) conducted a meta-analysis of 21 evidence-based treatments for children and youth exposed to traumatic events and found an average treatment effect size of .43 for post-traumatic stress symptoms, .25 for depressive symptoms, .09 for anxiety, and .18 for externalizing behavior problems when treatments were compared to active treatment, versus $.34, .22, .01$. , and .37 respectively when treatments were compared to wait-list control samples. Taken together, such 
evidence indicates the powerful impact of evidence-based treatment on a wide range of children's mental health difficulties.

\section{Weaknesses of Evidence-Based Treatment}

Despite established strengths, a variety of weaknesses of evidence-based treatment have also been noted. Some have critiqued evidence-based treatments on the grounds that they are so highly maualized as to be rigid and inadaptable to the individual needs of a child and family (Kendall., 1998; McNeil, Filcheck, Greco, Ware, \& Bernard, 2001). Additionally, others have noted that despite the overwhelming evidence supporting evidence-based treatments over treatment as usual models, a lack of dissemination of such treatments contributes to the fact that many youth continue to be treated with therapy backed by a lack of empirical support (Weisz, 2000). Finally, even when treatment dissemination and implementation occurs, some treatments have noted particular limitations concerning high levels of premature treatment drop out rates. For example, attrition rates in Parent-Child Interaction Therapy (PCIT) have been reported around 36\% in controlled efficacy studies (Fernandez \& Eyberg, 2009; Werba, Eyberg, Boggs \& Algina, 2006). However, drop-out rates in community effectiveness studies, including work in PCIT have been reported to be $60-70 \%$ (Pearl et al., 2012; Stokes, 2014). A range of factors including low family socioeconomic status, high levels of maternal critical speech, and lower levels of positive speech were found to be associated with higher levels of premature, treatment drop out (Fernandez \& Eyberg, 2009). Taken together, evidence-based treatment has been criticized for its structured treatment approach, lack of dissemination, and high attrition rates.

\section{Parent-Child Interaction Therapy}

PCIT theory. Parent-Child Interaction Therapy (PCIT; Eyberg \& Funderburk, 2011; McNeil \& Hembree-Kigin, 2010) is an evidence-based, behavioral intervention developed for 
young children with disruptive behavior disorders. Theoretically, PCIT is based on a number of models including attachment theory, social learning theory, and Patterson's coercion theory (Forgatch \& Patterson, 2003). Conceptualized using Hanf's two stage operant model (Hanf, 1969; Reitman \& McMahon, 2012) and Baumrind's (Baumrind, 1971) authoritative parenting style, caregivers are taught to balance positive, attentive caregiving responses with consistent, firm limits to foster a balance between parental warmth and control.

PCIT treatment. PCIT utilizes a live coaching model through a bug-in-the-ear device while caregivers interact with their 2-7 year old child on the opposite side of a one-way mirror. Therapy is divided into two phases: Child Directed Interaction (CDI) and Parent Directed Interaction (PDI). The focus of CDI lies in the development of a warm, positive, consistent relationship between the caregiver and child. During CDI, caregivers are instructed to follow their children's lead while increasing their use of positive verbalizations and decreasing their use of negative verbalizations. Caregivers are taught a series of "do" and "don't" skills to improve the quality of their interaction with the child. Appropriately labeled the "PRIDE" skills (i.e., praise, reflection, imitation, description, enjoyment), caregivers are taught to use specific labeled praises which involve providing a positive evaluation of a specific behavior or product of the child (e.g., "Thank you for sitting in your chair"), behavior descriptions which involve describing an action of the child (e.g., "You put the red block on top of the green block") and reflection which involves repeating the meaning of the child's speech back to him or her (e.g., child: "I drew a circle", caregiver "You did draw a big circle"). Simultaneously, caregivers are taught to avoid controlling the play and using negative verbalizations including questions (e.g., "What do you want to do?"), commands (e.g., "Sit down"), and critical statements (e.g., "Don't 
throw the toys"). Additionally, techniques such as selective attention are used to minimize negative interactions.

Caregiver-child interaction skills are practiced during 5 minutes of play-based homework assigned daily. Upon the initiation of each session, therapists code parents according to the Dyadic Parent-Child Interaction Coding System (DPICS), an empirically-based structured coding manual to measure the quality of the caregiver-child interaction (Eyberg, Nelson, Duke \& Boggs, 2005). Following the demonstration of mastery levels (10 specific labeled praises, 10 behavioral descriptions, 10 reflections and 3 or fewer questions, commands and critical statements) in a five-minute coded, parent-child interaction, caregivers are allowed to progress to the discipline phase of treatment, PDI.

In PDI, positive CDI skills continue to be utilized at high rates during caregiver-child play. However, caregivers are also taught to add effective commands to interactions with their children to increase children's compliance and decrease negative, attention-seeking behaviors. A standardized, time-out procedure is implemented following non-compliance to parental commands. Key tenants of PDI include consistency, predictability, and following through with parental demands. Initially, caregivers and children also complete 5 minutes of daily PDI homework designed to increase caregiver effective commands and children's compliance. As treatment progresses, the use of effective commands occurs in real world home and community settings to increase generalization. In order for a caregiver to master PDI, caregivers must issue $75 \%$ effective commands and demonstrate $75 \%$ effective follow through to effective commands in a 5-minute coded play sequence with the child. A course of PCIT typically lasts approximately 12-20 weeks, and graduation occurs once caregivers (a) report typical levels of child behavior 
difficulties according to the Eyberg Child Behavior Inventory (ECBI; Eyberg \& Pincus, 1999) and (b) report feeling confident in their ability to handle the child's behavior.

PCIT research. Kaminiski, Valle, Filene, and Boyle (2008) conducted a meta-analysis of programs to examine various training methods utilized in evidence-based parent training to decrease behavior problems in young children. Results indicated that parenting programs that required parents to practice positive parenting skills with the target child during treatment sessions had larger effect sizes than parenting programs without this component (Kaminiski et al., 2008). Additionally, parenting programs utilizing time-out as a disciplinary procedure for externalizing behavior problems resulted in larger effect sizes as compared to programs without this strategy. Finally, treatments utilizing the two-stage Hanf model in which parents first practiced positive reinforcement techniques, delivery of clear commands, and utilization of differential attention to increase appropriate behavior and decrease inappropriate behavior resulted in improved parent and child behavior and an improved parent-child relationship (Kaminiski et al., 2008; McMahon \& Forehand, 2003). PCIT includes each of the aforementioned components (McNeil \& Hembree-Kigin, 2010). Therefore, it follows that empirical research has demonstrated a strong evidence base validating the use of PCIT to decrease disruptive behaviors, increase the quality of the parent-child relationship, increase compliance, and improve child and caregiver functioning.

PCIT skills have been found to generalize to untreated siblings (Brestan, Eyberg, Boggs, \& Algina, 1997) as well as the target child's school environment (McNeil, Eyberg, Eisenstadt, Newcomb, \& Funderburk, 1991). PCIT has also been successfully adapted for a variety of diverse cultural groups including Latino (Borrego, Anhalt, Terao, Vargas, \& Urquiza, 2006; McCabe \& Yeh, 2009), African American (Capage, Bennett \& McNeil, 2001; Fernandez, Butler, 
\& Eyberg, 2011), Asian (Leung, Tsang, Heung, \& Yiu, 2009); Puerto Rican (Matos, Bauermeister \& Bernal, 2009) and Australian children (Phillips, Morgan, Cawthorne, \& Barnett, 2008). Furthermore, PCIT has been successfully modified to improve behavior difficulties and increase adaptive functioning in children with a variety of psychological diagnoses including attention deficit hyperactivity disorder (Matos, Bauermeister \& Bernal, 2009; Wagner \& McNeil, 2008), autism (Masse, McNeil, Wagner, \& Quetsch, 2016; Solomon, Ono, Timmer, \& GoodlinJones, 2008), separation anxiety disorder (Pincus, Santucci, Ehrenreich, \& Eyberg, 2008), selective mutism (Carpenter, Puliafico, Kurtz, Pincus \& Comer, 2014), trauma (Pearl et al., 2012) and major depressive disorder (Lenze, Pautsch, \& Luby, 2011). Furthermore, PCIT has been found to reduce child abuse recidivism as compared to an enhanced version of PCIT or community-based treatment as usual (Chaffin et al., 2004; Chaffin, Funderburn, Bard, Valle, \& Gurwitch, 2011). Furthermore, PCIT has been found to improve children's social skills (Chase \& Eyberg, 2008) and ability to regulate emotions (Timmer et al., 2011). The effects of PCIT have been found to extend up to six years following the conclusion of treatment (Hood \& Eyberg, 2003). Finally, a version of PCIT has been adapted to use with teachers with results indicating improvements in children's classroom behavior, increases in teacher's use of positive verbalizations, decreases in teacher's negative verbalizations, and less use of negative classroom consequences (e.g., time-out) following treatment (Lyon et al., 2009; Tiano \& McNeil, 2005).

PCIT effectiveness studies conducted to date have also demonstrated successful implementation across community-based settings (Beveridge et al., 2015; Fowles, Masse, McGoron, Beveridge, Williamson, Smith, Parish, in preparation; Leung, Tsang, Heung \& Yiu, 2009; Lyon \& Budd, 2010; Pearl et al., 2012; Self-Brown et al., 2012; Stokes, 2014) as well as in the home setting (Fowles, et al., in preparation; Galanter et al., 2012; Ware, McNeil, Masse \& 
Stevens, 2008). Results have indicated positive results across a number of child variables including child compliance, increased caregiver positive verbalizations, decreased caregiver negative verbalizations, and decreased child behavior problems. Taken together, results suggest that the positive outcomes of PCIT found in the clinic setting can also occur in more natural community-based settings, highlighting the robust effects of this evidence-based model.

Despite overwhelming evidence demonstrating the effects of PCIT on improving child behavior problems, significant implementation and delivery-based weaknesses have negatively impacted dissemination of the model, particularly high attrition rates (Fernandez \& Eyberg, 2009), costly initial training and treatment materials (Goldfine, Wagner, Branstetter, \& McNeil, 2007), and a lengthy certification process (PCIT International, 2016). However, given the relative lack of effectiveness research across PCIT studies and the broader parent training literature, such limitations have been primarily discussed in the context of clinic-based efficacy studies. In fact, Baumann et al. (2015) recently conducted a meta-analysis examining effectiveness research base of 610 cultural adaptation and implementation studies across four primary evidence-based parent training programs. Such interventions included: Parent-Child Interaction Therapy (PCIT), The Incredible Years (IY), Parent Management Training-Oregon Model (PMTO), and Positive Parenting Program (Triple P). All studies were required to meet implementation criteria detailed by the Cochrane Effective Practice and Organisation of Care Group (2002) and specifically include the use of one of four rigorous methodological designs. Across all four interventions, only two PCIT articles met standards, demonstrating the significant dearth of and need for rigorous evaluation and implementation of parent-training effectiveness research (Chaffin et al., 2009; Herschell et al., 2009). It is clear that more effectiveness work is 
needed to evaluate and successfully disseminate the PCIT model to children and families currently unable to access or successfully complete clinic-based treatment services.

\section{Effectiveness Methodology}

Effectiveness research is defined by the application of an efficacious treatment to realworld, applied settings (Chambless et al., 1998). Such research often purports strong external validity and weak internal validity relative to efficacy studies. Therefore, the target population of participants in effectiveness research, as compared to efficacy work, may seek treatment in a community clinic as compared to those recruited to a laboratory. Furthermore, treatment may be delivered by real-world, community therapists as compared to nonprofessional individuals trained for purposes of the research study. In effectiveness research, the therapist may decide how therapy should be conducted, whereas efficacy research may utilize a manualized protocol to guide treatment. Finally, assessment methods in effectiveness research may be conducted via an unobtrusive rating of the patient-determined problem behavior whereas a standardized measure of behavior problems may be used in efficacy work (Kazdin, 2003). While efficacy studies are often designed to determine the outcome of a procedure in a well-controlled setting, effectiveness research often carries high levels of generalizability to real-world settings.

Exploratory evaluations can be used in effectiveness research to further elucidate project goals and contribute to larger-scale designs. Small-sample studies, a type of exploratory evaluation, are often used prior to larger scale program designs to evaluate new behaviors or test the efficacy of a new measure. Small-sample studies can produce meaningful insight into the effectiveness of a current program or clarify the potential feasibility of conducting further studies (Wholey, 2015 in Newcomer, Hatry, \& Wholey, 2015). 
Multiple methodological approaches can be used when conducting an effectiveness study. For example, comparison group designs examine the impact of a treatment between two groups. Some designs are less likely to suffer from threats to internal and external validity (e.g., matching designs) than others (e.g., naïve design) and therefore, the results of such designs differ in their ability to determine causality (Henry, 2015 in Newcomer, Hatry, \& Wholey, 2015). Randomized controlled trials are known to minimize threats to internal validity by randomizing participants to groups, thereby attempting to minimize differences between groups prior to implementation of the manipulation (Torgerson, Torgerson \& Taylor, 2015 in Newcomer, Hatry, \& Wholey, 2015). Of course, smaller sample sizes may result in Type II error in which effects may be statistically insignificant but have important implications for policy. Therefore, the topic of study and impact of statistically insignificant differences should still be examined. In contrast to randomized designs, case studies provide an in-depth examination of a single or small group of programs. An exploratory case study uses a few select sources to provide insight into a broader question and are used to contribute to the development of a larger evaluation. While a descriptive case study might provide an in-depth description of a program, an explanatory case study would provide insight into the causes and effects within a given program.

The use of archival records provides a convenient and practical method to obtain a significant amount of useful information. Such records may be obtained directly from an agency, public database, or administration. Unfortunately, difficulties such as incomplete data, accuracy of information, and confidentiality concerns may negatively impact the quality of the evaluation. Similarly, surveys enable the evaluator to collect information from a large number of respondents (Hatry, 2015 in Newcomer, Hatry, \& Wholey, 2015). Surveys may be conducted in person, or using the internet, telephone or social media. Survey questions must be carefully 
constructed to avoid leading questions, and incentives for completion may be useful. Role playing is another methodological technique that may be used in effectiveness research to better understand a topic or determine the prevalence of an issue. Of key importance to future generalizability of such findings is the representativeness and size of the sample. Furthermore, role players must be carefully trained to implement the role play in a standardized fashion (Aranda, Levy, \& Stoney, 2015 in Newcomer, Hatry, \& Wholey, 2015). Another data collection technique involves the use of observers trained to determine a detectable characteristic of a program. Such a technique may be used in the context of a naturally occurring environment or following an intervention (Berman \& Vasquez, 2015 in Newcomer, Hatry, \& Wholey, 2015). Finally, focus group interviewing provides researchers with qualitative insight into a predetermined topic. Participants may include those directly involved in the study as well as those potentially impacted by its results. A knowledgeable and conscientious moderator is important to guide conversation and encourage meaningful participation (Krueger \& Casey, 2015 in Newcomer, Hatry, \& Wholey, 2015).

Effectiveness research may result in the use of qualitative as well as quantitative data analysis. Qualitative data provide a unique, detailed understanding of the experiences of individuals involved in a research program. Data may be coded using sophisticated programs or analyzed to determine thematic categories. Such themes may be transformed into numerical categories to be analyzed using quantitative methods. Furthermore, data may be analyzed descriptively using tables or hermeneutic methods to extract meaning or patterns. Finally, explanatory techniques attempt to determine theoretical cause and effect relationships between variables of interest. The particular method of choice is dependent upon the data collected and goals of the analysis. 
In conclusion, effectiveness research presents a methodological context by which to disseminate evidence-based treatment to children and families in real-world treatment settings. Research on wraparound initiatives typically require effectiveness methodology rather than efficacy tools, due to the community-based nature of the intervention.

\section{Combining Wraparound and Evidence-Based Approaches}

Researchers have compared the lack of research on wraparound services to the wealth of literature on evidence-based treatment. Some have noted that the rate of research on evidencebased treatment has far exceeded that of wraparound services (Suter \& Bruns, 2009). The two treatment frameworks have even been described as "different worlds of children's mental health" (Weisz, Sandler, Durlak \& Anton, 2006, p. 644). Given the clear strengths and weaknesses of these two treatment modalities, a call to integrate evidence-based research within wraparound systems has influenced work dedicated to combining both approaches (Suter \& Bruns, 2009). Multi-Systemic Therapy (MST), an empirically based intervention for serious juvenile offenders (Huey et al., 2000), combines a community-based, ecological perspective with evidence-based approaches (e.g., cognitive-behavioral treatments, parent training, behavior therapy) to reducing anti-social behavior and improving youth and family functioning (Eyberg, Nelson, \& Boggs, 2008; Henggeler \& Lee, 2003). Treatment teams may be comprised of multiple master's level therapists, a bachelor's level therapist, a medical doctor, and a principal investigator (Huey et al., 2000). Treatment occurs within the family as well as across community settings (e.g., school, community centers, sports teams) in which the youth participates. Primary outcome factors have included variables such as increased family cohesion, family functioning and family relations, as well as decreasing deviant peer relationships (Huey et al., 2000). In 2007, Stambaugh et al. conducted an empirical comparison between MST and wraparound. Results noted that children 
who received pure MST demonstrated greater clinical gains than those who had only participated in wraparound during the same 1.5 year time frame. The authors concluded that the inclusion of both effective and ineffective treatment components were likely included in the wraparound service while MST only included consistent, effective components (Winters \& Metz, 2009). Taken together, it appears reasonable to conclude that wraparound may benefit from more standardized, evidence-based components to increase treatment efficacy. 


\section{Part III: Feasibility}

\section{Pilot Study Definition}

Given the dearth of empirical evidence on wraparound services relative to PCIT, an initial evaluation to determine the viability of implementing an adapted, evidence-based approach within the wraparound system is needed. A pilot study represents one such preliminary investigation of a larger research question. The primary aim of pilot studies is to determine whether each of the components of the larger project will function effectively as a whole. Such evaluations are often underpowered. Results represent preliminary data used to refine key questions and methods used in larger implementation (Arain, Campbell, Cooper \& Lancaster, 2010). As a result of such small sample sizes, care should be used when drawing conclusions from such data (Kraemer, Mintz, Noda, Tinklenberg, \& Yesavage, 2006). Such limitations may contribute to a lower, overall rate of publication (Arain, Campbell, Cooper \& Lancaster, 2010). Typically, descriptive statistics serve as a primary form of data and the use of hypothesis testing is uncommon. Additionally, it is not advisable to combine data from the pilot investigation with that used in the primary research evaluation (Lancaster, Dodd \& Williamson, 2004). Pilot studies purport predetermined hypotheses and methodological procedures. Additionally, most forecast the intention of conducting further work. However, a small percentage actually follow through with publishing the results of their larger studies (Lancaster, Dodd, \& Williamson, 2004). An internal pilot study combines data from the initial pilot study with the remainder of the trial in outcome analyses. In contrast, if pilot data is collected and not used in the ultimate trial, it is labeled as an external pilot study (Arain, Campbell, Cooper \& Lancaster, 2010). It is more likely that pilot studies, as compared to feasibility research will have greater methodological rigor. For 
example, pilot studies may include a preliminary estimate of sample size and use randomization procedures and a control group (Arain, Campbell, Cooper \& Lancaster, 2010).

\section{Feasibility Study Definition}

Similar to pilot studies, feasibility studies are also used to help determine if an intervention can be effective on a larger scale (Bowen et al., 2009). Feasibility studies are typically larger than pilot studies, and it is commonly accepted that, while an explanation regarding sample size is important in both feasibility and pilot studies, an official analysis is not critical (Billingham, Whitehead, \& Julious, 2013). Questions such as "Can it work?", "Does it work"?, and "Will it work"? are central questions in feasibility research (Bowen et al., 2009, p. 454-455). Typically, feasibility studies do not analyze the primary outcome question, rather this question is typically reserved for the larger research project (Arain, Campbell, Cooper \& Lancaster, 2010).

The National Institute for Health Research (NHS) has identified variables that constitute key areas of evaluation in feasibility research (National Institute for Health Research, 2016). Feasibility variables may include potential for randomization of participants, recruitment of participants, availability and appropriateness of assessment measures, response rates, and duration of the study. Specifically, Bowen et al. (2009) note that feasibility research may be appropriate when 1) collaboration between researchers and community partners needs to be established, 2) little research exists with the specific population or intervention to be evaluated, and 3) previously employed methods have not contributed to successful implementation and the current evaluation intends to improve upon such methods. The primary focus of feasibility work may lie in understanding the acceptability of a given program, evaluating demand for a program, or determining the likelihood for its larger-scale implementation and use. Therefore, the specific 
aims of such work must operationalize success as well as outline the particular stages necessary to achieve such conclusions (C. Duncan personal communication, October 5, 2015). Feasibility studies may also be used to determine the practicality of implementation following considerations such as time, cost, potential for adaptation to a new format, or likelihood that the program could be successfully integrated within an existing system. Feasibility research may function to expand the use of a current program to a new context or to determine outcomes after methodological changes have been applied (Bowen et al., 2009).

Some debate regarding the differences between the definitions of pilot versus feasibility research exists (Arain, Campbell, Cooper \& Lancaster, 2010). Literature from the medical and health psychology disciplines have utilized a feasibility approach to assess whether an efficacy study should be conducted (Bowen et al., 2009). In feasibility research, a power estimate is not typically determined, although an appropriate number of participants should be present to enable researchers to evaluate feasibility variables. Overall, feasibility studies typically utilize fewer and less rigorous methodological procedures in contrast to pilot evaluations.

\section{Need for A Feasibility Approach in PCIT Effectiveness Research}

Feasibility studies serve a primary role in evaluating the potential for the larger scale design, evaluation, and implementation of a given research question. A specific need exists for feasibility studies conducted in community settings given the high proportion of research on evidence-based interventions conducted in controlled, laboratory environments (Bowen et al., 2009). Clearly, significant methodological and clinical weaknesses of the wraparound service model have limited its impact. Similarly, primary weaknesses of PCIT including the lack of effectiveness research, high attrition rates, and costly initial implementation have limited the scope of its dissemination, particularly among the complex cases most in need of treatment. A 
striking need exists to develop and test the implementation of a PCIT-based system of care within the existing structure of wraparound services. Labeled Staff-Child Interaction Therapy (SCIT), the current study examined the acceptability, implementation, and practicality of SCIT to better understand the feasibility of implementing the novel service within the wraparound systems of care. Specifically, feasibility will be operationalized by examining three key variables: 1) cost, 2) attrition, and 3) acceptability of the treatment. Strengths and limitations regarding integration and expansion of the novel approach within the wraparound system of care will also be examined to assist in illuminating areas of concern and future directions for larger implementation of the program.

\section{Cost: Wraparound Versus PCIT}

The costs associated with feasibility research vary depending upon the variables examined, length of the assessment, personnel involved, materials, and assessment methods used. For example, expenses such as therapist time and travel, training materials, facility costs, ongoing consultation, and trainer salaries must be included in the prospective budget. The Washington State Institute for Public Policy (Washington State Institute for Public Policy, 2015) has compiled a comprehensive list of empirically-based treatment programs spanning a wide breadth of disciplines including juvenile justice, child welfare, pre-k to 12 education, and children's mental health. Respective literature reviews were conducted and cost to benefit ratios were broken down into total benefits, taxpayer benefits, non-taxpayer benefits, and total costs. Finally, a net value of each treatment was determined as well as a benefit to cost ratio and percentage chance that benefits will exceed costs. A 2015 cost estimate determined that the benefit to cost ratio of PCIT was $\$ 13.68$ with a $95 \%$ chance that the benefits will exceed costs for families in child welfare and $\$ 2.23$ with a $78 \%$ chance that the benefits will exceed costs for 
children with disruptive behavior (Washington State Institute for Public Policy, 2015). Such cost findings were replicated by Goldfine, Wagner, Branstetter, \& McNeil, 2007 who estimated the cost of a single course of PCIT treatment to be $\$ 1000$ from intake to termination in addition to the $\$ 14,000$ initial start-up cost of a PCIT facility. Similarly, Krivelyova, Sukumar, Stephens, and Freeman (2007) estimated that treating a child with PCIT as compared to treatment as usual results in a savings of $\$ 600$ following 18 months of services (Krivelyova, Sukumar, Stephens, and Freeman, 2007 in Goldfine et al., 2007).

Various sources have also examined costs related to the implementation of wraparound service programs (Bazron, 2012; Bruns et al., 2010; Wraparound Milwaukee, 2013). Bickman and colleagues (2003) noted that wraparound services were more costly when compared to services as usual. Although estimates vary, an analysis by Bazron (2012) reported the cost of wraparound services to be approximately $\$ 27,000$ per individual, per year. Subsequent reports have estimated annual costs to be higher. An analysis conducted by Wraparound Milwaukee, a program noted to be one of the most long-standing and successful in the country, indicated a cost of $\$ 131$ per day for a youth in wraparound services (Kamradt, 2011). Based on a 365 day year, total costs for a youth in wraparound would amount to $\$ 47,815$. Additional estimates have indicated that a single youth in wraparound may cost $\$ 216$ per day or $\$ 78,840$ based on a full calendar year. Specifically, the Department of Human Services noted having served 199,076 children in its behavioral health services in the 2013-2014 fiscal year. The total cost to serve such children was $\$ 1,221,933,564$, or $\$ 6,138.03$ per child (Pennsylvania Department of Human Services, 2015). Such costs are often justified by comparing wraparound to the cost of higher levels of care such as juvenile detention and residential treatment. In such restrictive environments, costs may range from $\$ 150,000$ (Bazron, 2012) for a single stay to $\$ 352,663$ per 
year, per offender in New York (Justice Policy Institute, 2014). Across the United States, approximately $\$ 7.1$ million dollars per day is spent on juvenile detention services (Justice Policy Institute, 2014). Despite clear savings when compared to higher, more intensive levels of care, the costs of wraparound pale in comparison to those incurred from the implementation of PCIT.

\section{Attrition}

Attrition represents a primary variable of interest in feasibility research as the retention of participants is critical to the stability of an intervention and therefore, the likelihood that participants may benefit from its implementation. Broadly, attrition in research is primarily problematic as it may result in biased groups that no longer represent the population of interest and may compromise attributions of causality (Amico, 2009). Given that a primary aim of many feasibility studies is to inform the potential development of a larger research program, some feasibility work specifies predefined levels of acceptable attrition, recruitment, and measure completion in order for a trial to advance to a larger investigation (e.g., $<20 \%$ ) (Sniehotta et al., 2011).

Although the precise operationalization of attrition varies across feasibility studies (Sniehotta et al., 2011), many examinations utilize CONSORT flowcharts and tables to illustrate recruitment, retention and, attrition rates between groups throughout study procedures. Previous work has specifically examined attrition within hosted interventions (Amico, 2009). Defined as "an intervention ... offered within or in conjunction with existing community, health, or private services" (Amico, 2009, p. 1568), a hosted intervention must consider two forms of attrition, that of the research participants and that of individuals in the larger network outside of the study analysis. Therefore, such interventions may risk high rates of attrition given its interdependence upon attendance nested within the larger system. In such interventions, participant attrition may 
occur in multiple forms including 1) participants who drop out of an intervention following partial completion, 2) participants who finish an intervention but whose data includes missing responses and 3) participants who do not receive any dose of an intervention (Amico, 2009). Therefore, a thorough understanding of attrition must include an analysis of differential forms of and reasons for attrition between both treatment and control groups (Amico, 2009). Such analysis allows researchers to examine patterns of attrition between groups and address potential methods for successful recruitment and retention of previously unsuccessful participants in future research (Amico, 2009; Sniehotta et al., 2011). An analysis of differential attrition between groups is of critical importance to feasibility research conducted using hosted intervention designs as such examination lends support to the utility of including a treatment within a larger, previously established system and informs subsequent research.

\section{Acceptability}

Finally, research examining the acceptability of a new treatment is commonly studied in the context of feasibility research to better understand participants' reactions to a novel service and therefore its potential for utilization in larger scale implementation (Bowen et al., 2009; Sniehotta et al., 2011). Specifically, procedures related to participant recruitment, assignment, assessment, and retention may be modified to improve the implementation and delivery of the treatment in preparation for a larger study (Sniehotta et al., 2011). To better understand acceptability, motives for attrition may be analyzed and such reasons may be contrasted between conditions. Fidelity to treatment measures may also be used to gauge the acceptability of an intervention. A questionnaire inquiring about participant's thoughts and feelings toward the intervention can also inform its acceptability (Bowen et al., 2009). In fact, it has been argued that in order to effectively narrow the gap between research and community practice, ongoing 
collaboration between evaluators and community stakeholders is critical (Frazier, Formoso, Birman, \& Atkins, 2008). In an attempt to operationalize feasibility, Frazier, Formoso, Birman and Atkins (2008) argue that not only must evaluations consider access to a given population but they must also determine key service providers, environments where services will be provided, and the funding agent of services over time. Therefore, the partnership between researchers and community-based agencies serve a foundational role in the execution of feasibility work. Essentially, feasibility evaluations must determine if and how a given program could produce maximum impact within the context of an existent system so that the program could be successfully integrated into the company and sustained over time (Kingston, 2004).

Sense of therapist competence. Therapist competence has been defined as, "the extent to which a therapist has the knowledge and skill required to deliver a treatment to the standard needed for it to achieve its expected effects" (Fairburn \& Cooper, 2011, p. 374). Generally, previous literature suggests mixed effects of therapist competence on treatment outcome (Shaw et al., 1999; Trepka, Rees, Shapiro, Hardy, \& Barkham, 2004; Webb, DeRubeis, \& Barber, 2010). Although a lack of empirical research exists on the effect of therapist competence on behavioral parent training outcomes, the broad research literature examining the impact of therapist competence on cognitive and cognitive-behavioral treatment modalities have been explored. For example, Webb, DeRubeis, \& Barber, 2010 conducted a meta-analysis of 36 studies examining the role of therapist competence on client symptom change. Studies included in the review met strict inclusion criteria including examinations of individual psychotherapy, quantitative data of therapist competence, and expert ratings of recorded therapy sessions. Results indicated that therapist competence was not related to client outcome, however, the authors indicate that stronger relationships between therapist competency and outcome were 
present among investigations of depressed clients or when the influence of the therapeutic alliance was not controlled for in analysis. Additional research has demonstrated some support for the influence of therapist competence on depressed patient treatment outcomes, particularly among therapists with a primarily cognitive and cognitive-behavioral treatment orientation (Shaw et al., 1999; Trepka, Rees, Shapiro, Hardy, \& Barkham, 2004). Taken together, some evidence exists for the impact of therapist competence on client outcomes.

More recent work has begun to examine the most effective methods by which therapists learn to effectively deliver psychological treatments (Fairburn \& Cooper, 2011). Beidas and Kendall, 2010 conducted a comprehensive literature review of the outcome of various training approaches of evidence-based, mental health service providers published between the years 1990-2008. To be included, studies must have examined therapist variables such as previous clinical experience or theoretical orientation, a measure of organizational factors, a description of the training characteristics, and a measure of client severity. Studies were classified as either including passive training approaches (e.g., didactic presentation, lecture) or active learning (e.g., behavioral role-play, feedback, coaching). Results indicated that changes in therapist competence and client outcomes only occurred when trainings addressed each area of the systems-contextual model (i.e., "quality of the training, practitioner variables, client variables, and organizational support”, Beidas \& Kendall, 2010, p. 2) and included active learning components. Such results indicate the importance of and potential to affect change in therapist behavior and competence during training of evidence-based practices through active learning strategies.

Despite powerful training methods, some debate continues to exist regarding the method by which therapist competence and quality of treatment are assessed. Namely, direct assessment of therapist knowledge, therapist skill, client outcomes, and assessment of actual therapy 
sessions have each been proposed as potential targets of therapist competence and skill (Fairburn \& Cooper, 2011). Each method includes inherent strengths and weaknesses including cost, a lack of standardized measures, assessment of variables other than the treatment itself, and limitations of the Hawthorne effect whereby performance improves when individuals are watched. Although promising findings regarding the effects of active training methods have resulted in positive changes in therapist knowledge and skill, ongoing work continues to examine the precise methods by which therapist competence is operationalized and measured.

\section{The Current Study: Staff Child Interaction Therapy}

Overview and history of SCIT. Given the critical need for the use of high-quality, empirically-based treatment approaches within the framework of in-home wraparound services, the current study will have two primary purposes: 1) to examine primary changes in child behavior problems between children receiving SCIT as compared to those receiving treatment as usual and (2) to evaluate the feasibility of implementing PCIT-based skills as a novel form of service delivery in wraparound treatment.

Originally proposed by Gus Diamond (McNeil \& Hembree-Kigin, 2010), the current SCIT model is based off of its original (1999) inpatient implementation by Dr. Toni HembreeKigin, $\mathrm{PhD}$ in an inpatient child crisis center for children aged 4-12 years. A baseline assessment was conducted indicating that the staff utilized few positive verbalizations during a one-hour play-based observation with the group. In-person and video-based didactic sessions were conducted to teach the SCIT skills. Coaching sessions occurred on the unit such that staff members learned to implement SCIT skills with small groups of children. Although this was a descriptive case study of the intervention and numerical results were not presented, a summary of treatment conclusions indicated that upon the conclusion of treatment, the frequency of 
positive staff verbalizations increased and few consequences (e.g., redirection, time-out) were needed to manage children's behavior in a free-play environment. Staff also reported feeling less stressed and more satisfied with their jobs, as well as perceiving themselves as having better relationships and greater confidence in their work with the children.

Such results reflect outcomes of a similar, recently developed research-based model entitled Child-Adult Relationship Enhancement (CARE; Gurwitch, Messer, Masse, Olafson, Boat, \& Putnam, 2015) used with adults who come in contact with children at risk for maltreatment and subsequent emotional and behavioral problems. Unlike PCIT, the CARE model is considered nontherapeutic and therefore, does not specifically target severe aggression or behavioral challenges. Rather, the preventative approach has been used to train medical personnel as well as psychologists, child protection staff, educational providers, military personnel, and early childcare staff with a primary goal of increasing the positivity of any adultchild relationship and subsequently improving child compliance (Gurwitch et al., 2015). Although comprehensive research evaluating the model is in progress, over 100 trainees from a diversity of medical and home-service disciplines indicated extremely high levels of satisfaction with the model in addition to the belief that the training would impact the way they interact with youth (Gurwitch et al., 2015).

The current evaluation of Staff-Child Interaction Therapy is operationally distinct from the Gurwitch et al. (2015) CARE model and previously described model of SCIT proposed by Gus Diamond (McNeil \& Hembree-Kigin, 2010) in four primary ways. 1) It includes both a CDI relationship enhancement phase and a modified PDI to allow limit setting, 2) Although it teaches adults to use skills directly with children, it includes an important additional component, the transfer of such skills to the parents, 3) It directly teaches skill transfer with modeling, coding, 
tracking progress toward mastery, and providing frequent and systematic feedback to parents, 4) It is conducted in the homes of families in wraparound due to extremely challenging behavior. The Current Implementation of SCIT

A description of SCIT. The treatment model was developed and manualized by a team of researchers at West Virginia University (Norman \& McNeil, 2015). Primarily designed for children between 2-9 years of age with disruptive behavior difficulties (e.g., compliance, aggression), the 215 page SCIT manual is comprised of an overview of SCIT and 18 sessions of step-by-step protocol developed to increase treatment fidelity (Norman \& McNeil, 2015). Sessions one and two are delivered by a master's level therapist with the primary caregiver, however the child is involved in some aspects of the session. During such sessions, master's level therapists primarily work with the parent using techniques such as modeling, role play practice, and feedback to teach parents SCIT skills. See Appendix A for a description of a typical SCIT session.

Sessions three through 18 are conducted by the bachelor's level therapist with both the caregiver and child present. Bachelors-level staff are consistently supervised by master's level staff throughout the SCIT therapy course. Similar to PCIT, sessions are designed to be delivered for one hour as often as the therapist enters the home (up to five times per week). Caregivers also are expected to practice SCIT skills during daily homework sessions that occur when therapists are not present. Parents are told to practice skills with the child for a total of 10 minutes each day and record completion of their homework on a homework sheet. Similar to PCIT, SCIT is divided into two phases, Child Directed Interaction (CDI) and Adult Directed Interaction (ADI). The goal of CDI is to build a more positive, warm, consistent relationship between the caregiver and child. The goal of ADI is to improve the caregiver's ability to effectively discipline the child. 
To reach mastery of CDI, a caregiver must deliver 10 specific labeled praise statements, 10 verbal reflections, 10 behavioral descriptions, demonstrate enjoyment and imitation during play and issue no more than 3 questions, commands, and critical comments during a 5-minute coded caregiver-child interaction. Additionally, caregivers must demonstrate appropriate use of selective attention toward the child should instances of minor, attention seeking misbehavior arise. To reach mastery of ADI, the caregiver must deliver $75 \%$ effective commands during a play-based interaction and demonstrate $75 \%$ effective follow through to effective commands.

A rationale for SCIT versus PCIT. SCIT was developed as an alternative to PCIT to fill a gap in mental health treatment services for implementation by bachelor's level wraparound staff. SCIT was developed for a number of key reasons: (1) A greater number of bachelor's level staff perform in-home wraparound treatment with low-resource families as compared to master's level therapists, (2) bachelor's level therapists are less costly to managed care insurance companies than masters level clinicians, and (3) SCIT represents a lower- level intervention designed to decrease disruptive behaviors and increase therapist and parent skill prior to advancement to a higher-level, more costly intervention.

A number of key modifications were made to PCIT when developing Staff-Child Interaction Therapy (SCIT) for bachelor's level staff working within a wraparound framework (See Appendix B and the following section). When developing SCIT, significant modifications to the original PCIT protocol were made for many reasons. First, Parent-Child Interaction Therapy International guidelines (Parent-Child Interaction Therapy, 2016) state that in order for a therapist to practice PCIT, therapists must possess at least a master's degree in a mental health field and be a licensed mental health practitioner (or be working under the supervision of a licensed practitioner) or be at least a third year doctoral level psychology student under 
supervision by a licensed mental health practitioner. In addition, certified PCIT therapists must complete 40 hours of in-person training by a PCIT trainer or 10 hours of online training and 30 hours of in-person interaction with a PCIT trainer. Certified PCIT therapists must complete a minimum of 2 cases and participate in twice monthly consultation with a certified PCIT trainer for one year. Finally, therapists must have select PCIT sessions observed and approved by a certified PCIT trainer. Given that all of the therapists in the current evaluation were bachelor's level therapists, they do not meet the minimum requirements to deliver PCIT.

Additionally, the PCIT procedure involves a complex time-out procedure in which the parent may physically move the child to the time-out chair should he or she resist the procedure. Given that the home environment is an uncontrolled space in which the child's behavior may escalate to high levels of aggression and defiance, it would be considered dangerous for a bachelors level therapist to attempt to implement a time-out procedure in this setting. Therefore, a low-risk, primarily hands-off discipline procedure was designed in accordance with Pennsylvania state law to replace the hands-on time-out sequence. Learning to appropriately coach a time-out procedure, as conducted in PCIT involves higher level credentials and training than bachelors-level therapists possess (Parent-Child Interaction Therapy, 2016). Therefore, the SCIT protocol was designed as a low-risk alternative to PCIT which combines evidence-based relationship and discipline skills in a structured treatment package for bachelors-level therapists to implement in the home setting with caregivers.

Key differences between PCIT and SCIT. Differences between PCIT and SCIT are as follows: (1) SCIT is delivered in the home setting either on a small blanket space or in an enclosed room without the use of a one-way mirror or bug-in-the-ear device typically used in PCIT, (2) unlike PDI, Adult-Directed Interaction (ADI) does not involve time-out or physical 
removal of the child client, (3) the SCIT therapist does not provide any real time coaching of the parent, only modeling, coding, tracking, and feedback, (4) given the Pennsylvania state regulation stating that therapists cannot be alone with a child, parents were to remain in the room with the therapist and child on all occasions, (5) a restriction of privilege consequence replaced the time-out sequence for non-compliance to caregiver commands, (6) caregivers simultaneously worked toward mastery of both CDI and ADI during each session to maximize exposure to the relationship enhancement and discipline goals of SCIT, (7) treatment sessions occurred for one hour each time the staff member enters the home even if the staff member remains in the home for a longer period, (8) similar to PCIT, SCIT caregivers are assigned daily homework practice, but ADI homework practice does not generalize beyond effective commands in play situations, and (9) treatment concludes following 18 sessions or a predetermined 6-8 week interval (whichever occurs first) regardless of parental mastery or skill acquisition. See Appendix C for a table of specific similarities and differences between PCIT and SCIT.

\section{Purpose of the Present Study}

The purpose of the present study was twofold. First, the study examined the effectiveness of SCIT by measuring changes in child behavior problems in child clients assigned to SCIT as compared to those assigned to a treatment as usual attention control condition. Child behavior problems were examined from the perspective of the parent as well as the therapist. Second, an analysis of the feasibility of implementing SCIT as a new service within an existent wraparound framework was examined. Specifically, feasibility was operationalized by analyzing the costs related to SCIT implementation, attrition rates associated with SCIT, and finally, acceptability of the treatment among participating therapists. 


\section{Study Hypotheses}

Hypotheses. Children's behavior problems were expected to decrease more following SCIT training than following an attention control workshop.

Empirical evidence suggests that PCIT improves children's behavior by increasing children's compliance, decreasing aggression, and increasing children's social skills (Brestan, Eyberg, Boggs, \& Algina, 1997; Chaffin, Funderburk, Bard, Valle, \& Gurwitch, 2011; Matos, Bauermeister \& Bernal, 2009; McNeil, Eyberg, Eisenstadt, Newcomb, \& Funderburk, 1991). Given that SCIT is based off of evidence-based PCIT skills, it is expected that parents and therapists of children receiving SCIT will report decreased behavior problems as compared to therapists of children receiving treatment as usual.

Hypothesis 1: It is expected that TSS in the SCIT group will report a greater decrease in the intensity of child behavior problems compared to the TSS in a treatment as usual condition.

Hypothesis 2: It is expected that parents of children in the SCIT group will report a greater decrease in the intensity of child behavior problems compared to parents of TSS in the treatment as usual condition.

Feasibility Questions. How feasible is SCIT as an intervention service within the wraparound system of care model?

Rationale. Feasibility research commonly examines treatment cost (Bazron, 2012; Washington State Institute for Public Policy, 2015), attrition (Amico, 2009; Sniehotta et al., 2011), and acceptability (Bowen et al., 2009; Frazier, Formoso, Birman, \& Atkins, 2008 Kingston, 2004; Sniehotta et al., 2011) to determine the practicality of further study with a novel treatment. The current study examined the feasibility of implementing SCIT as a treatment for 
child behavior within the wraparound system of care by evaluating cost, attrition, and acceptability.

Specific Aims of Feasibility Assessment

a) Cost

a. What costs are associated with the implementation of SCIT within a wraparound system of care?

b) Attrition

a. Do significantly more therapists drop out of SCIT as compared to TAU prior to completion?

b. Do significantly more families drop out of SCIT as compared to TAU prior to completion?

c. Qualitatively, why does therapist attrition occur in the SCIT and TAU groups?

d. Qualitatively, why does client attrition occur in the SCIT and TAU groups?

c) Acceptability

a. Do SCIT and TAU therapists significantly differ in their reports of satisfaction with the trainings?

i. What do SCIT therapists perceive to be the qualitative strengths and weaknesses of the SCIT workshop?

ii. What do TAU therapists perceive to be the qualitative strengths and weaknesses of the attention control workshop (compassion fatigue)?

b. Hypothesis 3: It is expected that therapists in the SCIT group will report a greater increase in therapist competency compared to therapists in the treatment as usual condition. 
c. What type of qualitative feedback do TAU therapists provide regarding their experience implementing TAU in a wraparound system of care?

i. What qualitative themes emerge from focus group discussions with TAU therapists?

d. What type of qualitative feedback do SCIT therapists provide regarding their experience implementing SCIT in a wraparound system of care?

\section{Method}

\section{Participants and Setting}

Data was collected through Family Behavioral Resources (FBR), a community mental health agency based in Pittsburgh, Pennsylvania. The agency includes outpatient mental health clinics serving children, families, adults, and geriatric populations. Simultaneously, the for-profit agency also conducts wraparound treatment services through Behavioral Health Rehabilitative Services (BHRS) for children with severe emotional disorders and their families. FBR is made up of a single corporate office where company-wide decisions are made. The company also includes satellite offices across 21 counties around Pennsylvania. A typical FBR satellite office is organized in a hierarchical fashion whereby each subsequent individual supervises those under him or her. Depending upon the size of the office, a given branch may include a single masterslevel (i.e., MA, MSW, LSW) clinical director, 2-3 clinical supervisors, 4-6 master's-level behavior specialist consultants (BSC) and 15-40 bachelors-level therapeutic support staff (TSS). Upon completion of a higher educational degree, staff may progress through each subsequent ranking.

A BHRS service team includes a bachelor's level therapist, a master's level supervisor, and a site-based clinical director. The bachelor's level staff member, called therapeutic support 
staff (TSS), is responsible for delivering treatment to a caseload of approximately 4-5 child clients and their families. Each behavioral specialist consultant (BSC), a master's level therapist, is responsible for developing treatment goals, a treatment plan, and tracking behavioral data across sessions. Treatment sessions are delivered in the family's natural environment, namely the home, school, and community settings, to increase generalization of clinical gains. A single session may last approximately 1-3 hours depending upon the insurance-approved prescription. Although therapeutic support staff are primarily in charge of delivering treatment, the behavior specialist consultant may accompany the therapeutic support staff to the client's home to review a treatment plan, discuss goals, administer assessment measures, supervise the therapeutic support staff, or speak with the caregiver.

\section{FBR Offices Used in the Current Study}

Participants in the current study included 73 TSS, although drop out occurred throughout treatment. Therapists from each of the following nine BHRS satellite offices participated in the study: Uniontown, Washington, Latrobe, North Huntingdon, North Hills, Indiana, Brentwood, Robinson, and Monroeville.

\section{Inclusion/Exclusion Criteria}

To be enrolled in the current study, a child must have 1) been between 2-9 years old upon initiation of participation, 2) displayed disruptive behavior difficulties in one or multiple environments, (e.g., aggression, non-compliance) although such difficulties may not have been the primary diagnosable condition (e.g., child may have been in services for autism spectrum disorder or trauma), 3) received home-based BHRS services for at least 1 hour/week, 4) expected to remain in services for at least 4-6 months following initial participation in the study, and 5) been able to understand one-step commands (e.g., "please hand me the crayon"). Additionally, 
the associated therapist must have expected to remain with the agency for 4-6 months following initial participation. Child clients were excluded if the child did not speak English. Potential participants who did not meet these minimum criteria were excluded from the study. Workshops were advertised with the inclusion and exclusion criteria clearly specified.

\section{Participant Selection}

It should be noted that a change in agency administration occurred between the original planning phase and actual SCIT implementation phase approximately one month prior to initiation of the current project. New personnel had not been involved in previous research conducted between the current research team and BHRS, leading to confusion surrounding the impact of SCIT implementation on BHRS services as usual.

In light of such changes, the initial project was presented to the corporate office of FBR at a planning meeting. Agency corporate offices contacted the clinical director of each regional office, who notified therapists of the opportunity during staff meetings. The corporate offices, clinical directors, and therapists either directly contacted the researcher or provided the researcher with contact information of interested therapists. Specific therapists were encouraged to attend the workshops by their employer if a child on their caseload fit workshop criteria. Although a single TSS may have multiple child clients, each TSS in the current study chose one child with whom to implement SCIT. In the case that multiple children on a therapist's caseload fit study criteria, therapists were instructed to choose the child with the most severe behavior problems to include in the study. All other child clients on the therapist's caseload continued to receive treatment as usual and were not included in the study. Therefore, child clients in the current study had a single, consistent study-related therapist throughout implementation of the project. However, some of these children may have had multiple TSS working with them. For 
example, a single child may have one TSS in the home environment (participating in the current study) and a different TSS in the school environment. If a given child client discontinued BHRS services or decided not to receive SCIT part way through the study (e.g., between the first and second workshop but prior to SCIT implementation) and the therapist had a second, SCITeligible client on his or her caseload, therapists were allowed to switch clients and begin implementing SCIT with the new, eligible client. Therapists earned continuing education credit through their agency and were compensated by FBR for their travel and time.

The BSC of each TSS was also trained in SCIT in order to appropriately supervise their respective TSS in SCIT implementation. Such trainings lasted one session and were either conducted in conjunction with the TSS upon attendance to the first workshop or in a separate, day-long, BSC-only workshop. BSC's were not considered study participants and therefore, no study-related data were collected from them.

\section{Participant Assignment to Condition}

Therapists were assigned to a condition in one of two ways. First, when therapists were available for multiple workshop days, they were randomly assigned to either SCIT or TAU using a flip of a coin. Second, when therapists were available for only one workshop date, they were assigned based soley on availability without knowledge of the type of workshop being provided on that date. During therapeutic support staff consent procedures, therapeutic support staff learned that participation in the research project was voluntary and could be discontinued at any time. Additionally, TSS learned that they could choose to attend the SCIT or attention control (compassion fatigue) workshop without participation in the research study. TSS were informed that they could choose not to participate in the research without negative job-related 
consequences. See Table 1 for a flow chart describing participant recruitment, enrollment, and assignment to condition between groups (Amico, 2009).

\section{Measures}

Eyberg Child Behavior Inventory (ECBI; Eyberg \& Pincus, 1999). The Eyberg Child Behavior Inventory (ECBI; Eyberg \& Pincus, 1999) is a 36 item measure of child behavior problems for children between the ages of 2-16 years. In the current investigation, the ECBI was completed by both the TSS and the child client's primary caregiver. Parent ECBI data were obtained from the agency (FBR) and collected as part of the agency evaluation of SCIT. Through this evaluation, BSC's obtained the ECBI from the parent. An IRB addendum was submitted and deidentified ECBI data were approved to be shared with WVU research personnel at three points (i.e., following the first workshop, before the second workshop, and before the third workshop) during the workshop sequence. Only the intensity score was analyzed.

The measure includes two subscales: (1) an intensity scale in which the respondent rates how often the behavior occurs on a 7-point likert scale, and (2) a problem scale in which the respondent rates if the behavior is problematic for him or her on a dichotomous yes/no rating scale. Previous psychometric research indicates that the measure has high levels of validity and reliability. Specifically, Cronbach'a alpha scores of .95 for the intensity subscale and .93 for the problem subscale indicate that the measure possesses a high level of internal consistency (Colvin, Eyberg, \& Adams, 1999). Additionally, test-retest reliability statistics of $r=.75$ for the intensity scale over a 10 month time frame indicate that results of the measure remain stable over time (Eyberg \& Pincus, 1999).

High levels of concurrent validity have been achieved by comparing results of the ECBI to the Child Behavior Checklist (CBCL; Achenbach \& Rescorla, 2000), a similar standardized 
measure of child behavior problems. Results indicated that the ECBI intensity $(r=.75)$ and problems scales $(r=.67)$ were highly correlated with the CBCL externalizing subscale (Boggs, Eyberg, \& Reynolds, 1990) as well as the Parenting Stress Index (PSI; Abidin, 1990). Finally, discriminative validity of the ECBI has also been established by comparing results of the ECBI to samples of neglected and non-referred children (Aragona \& Eyberg, 1981; Funderburk, Eyberg, Rich \& Behar, 2003).

Psychometric data of the ECBI indicate that children in the standardization sample scored an average of $96.6(S D=35.2)$ on the ECBI as compared to a group of children diagnosed with ADHD, ODD and CD $(M=138.67, S D=28.48)$. Therefore, the cutoff score used on the ECBI intensity scale to determine clinically significant behavior problems is 131 .

Demographic questionnaire. Two demographic questionnaires (see Appendix D) were administered to the TSS at each of the three workshops. Basic demographic information such the sex, race, gender, educational level of the therapist, as well as more specific questions inquiring about previous therapist experience were included. Additionally, therapists reported key demographic variables for their clients, including child's length of time in mental health services, CPS involvement, and known mental health diagnoses.

Workshop feedback form. Following each workshop, therapists completed a satisfaction survey (see Appendix E). Survey items included a quantitative five-point likert scale and two qualitative, open-ended questions in which therapists indicated the strengths and weaknesses of the workshop.

Final SCIT survey. Following completion of SCIT, SCIT therapists completed a qualitative, open-ended questionnaire inquiring about strengths and weaknesses of SCIT as an intervention and the impact of SCIT upon themselves as a therapist (see Appendix F). 
The staff sense of competence scale (SSOC; Gibaud-Wallston \& Wandersman, 1978

in Johnston \& Mash, 1989). The staff sense of competence scale was adapted from the Parenting Sense of Competence Scale for bachelors-level, in-home therapists. The PSOC was originally developed as a 17-item scale to examine parents' level of self-esteem (GibaundWallston \& Wandersman, 1978 in Johnston \& Mash, 1989). A six point scale is used to rate items from strongly disagree to strongly agree. Measures of internal consistency were determined to be high for both the satisfaction (.82) and efficacy (.70) scales. Additionally, testretest reliability correlations fell in a range between .46 and .82 (Gibaund-Wallston \& Wandersman, 1978 in Johnston \& Mash, 1989). Standardization of the PSOC using a sample of mothers and fathers of 4-9 year old children, indicated that participant responses loaded on two primary factors, satisfaction indicating parents' feelings of frustration, anxiety, and motivation and efficacy, indicating parents' competence, problem-solving, and feelings of capability as a parent (Johnston \& Mash, 1989). More recent investigations of the SSOC have revealed an additional factor, interest, indicating parents' level of commitment to the position (Gilmore \& Cuskelly, 2008). See Appendix G for the SSOC.

\section{Procedure}

Overall study design. The evaluation of SCIT in the current study involved the use of a quasi-experimental, pre-post, attention control group design. Therapists in the present study attended three workshops, evenly spaced, seven weeks apart. The first and second workshops for both the SCIT and treatment as usual conditions occurred for eight hours between the hours of $9 \mathrm{am}-5 \mathrm{pm}$ with a one hour break for lunch. The third workshop occurred for 3 hours between the hours of $9 \mathrm{am}-12 \mathrm{pm}$ for both groups. Following the day-one SCIT training entitled, therapist skill mastery workshop, SCIT therapists were instructed not to begin the SCIT protocol but instead, 
use the play therapy skills taught during the workshop in individual sessions with their child clients. Following the day two SCIT training, entitled parent skill mastery workshop, SCIT therapists began using the SCIT protocol with parents and children. Implementation of SCIT concluded upon the third SCIT workshop. Treatment as usual therapists did not learn about or conduct SCIT. See Appendix H for a timeline of workshops for both groups.

SCIT day-one workshop: Therapist skill mastery. Therapeutic support staff in the SCIT condition attended an initial, 8-hour, SCIT workshop. Workshops took place at either the West Virginia University Life Sciences Building or at one of the FBR regional offices. Workshops were primarily led by the researcher with assistance from the remainder of the research team (approximately 2 graduate and 6 undergraduate students). Groups of approximately 10-15 therapists attended each workshop. See Appendix I for detailed schedules of each of the three days of SCIT and treatment as usual workshops. The SCIT day-one workshop began with therapist and staff introductions, a PowerPoint welcome presentation introducing the broad purpose of the study and past research conducted in the lab that led to the development of the current project. A rationale for SCIT was presented including differences between SCIT and PCIT and a consent form was explained. A pre-treatment assessment session lasting approximately 90 minutes then occurred and consisted of therapists completing selfreport measures, including the following: The Eyberg Child Behavior Inventory (ECBI; Eyberg \& Pincus, 1999) and two demographic questionnaires assessing therapist and child demographic characteristics (see Appendix D). Therapists were asked to play with an undergraduate student trained as a standardized child in two, 5-minute simulated play situations. During the first situation, the therapist was asked to allow the child to lead the play and in the second situation, the therapist was asked to tell the child to clean up the toys without the therapist's assistance. 
Graduate and undergraduate coders trained to reliability in the Dyadic Parent Child Interaction Coding System (DPICS; Eyberg, Nelson, Duke, \& Boggs, 2005) coded therapist verbalizations during such situations. An additional evaluator served as a reliability coder to ensure coding accuracy. This behavioral observation measure was included as part of a separate research study and the data were not evaluated in the current project.

Next, a 60-minute powerpoint presentation focusing on the first phase of SCIT, Child Directed Interaction (CDI), was presented. Specifically, CDI skills were composed of labeled praise, reflection, imitation, description and enjoyment, along with the use of selective attention. WVU staff modeled CDI and selective attention skills. WVU staff also discussed the DPICS. A rationale for the coding system was presented and examples of each code were provided. Therapists were verbally quizzed on various DPICS codes. To develop CDI skills, therapists were divided into groups of 3-4 therapists and 2-3 WVU staff to practice CDI skills, selective attention, and DPICS coding to mastery levels. Specifically, mastery of skills involved using 10 labeled praises, 10 behavior descriptions, 10 reflections, and three or fewer questions, commands and negative talk during a 5-minute simulated play situation. To master DPICS coding, therapists had to achieve $80 \%$ reliability with a WVU master coder during a 5-minute simulated play situation. Practice sessions occured for approximately 60 minutes.

Following lunch, a 45-minute powerpoint presentation focusing on the second phase of SCIT, Adult Directed Interaction (ADI), was presented (see Appendix J for ADI discipline sequence). Specifically, therapists learned the rules of giving effective commands. Upon noncompliance, therapists were taught to use a broken record technique in which they repeated the effective command three times. If the child complied, the therapist issued a contingent labeled praise following compliance. If the child did not comply following three identical 
commands, a warning statement was issued (i.e., "you have two choices, you can either [insert command] or I will help you [insert command]"). Upon noncompliance, a 5-second physical guide (i.e. hand over hand prompt) was utilized. Upon resistance to the physical guide, the therapist issued another warning statement (i.e., "you have two choices you can either [insert command] or you will lose [insert previously determined privilege]"). Upon noncompliance, the therapist told the child that he or she lost the privilege (i.e., "You did not [insert command] so you will lose [insert previously determined privilege]"). WVU staff modeled ADI skills for therapists. Therapists and staff then returned to their small groups to master ADI skills and continue to work towards mastery of CDI skills. Practice sessions occurred for approximately 60 minutes. A final question and answer session then occurred as a large group. Therapists reflected on the workshop and completed a workshop satisfaction form. Finally, SCIT therapists were asked to give their BSC supervisors the ECBI form. Instructions were included to ask BSC's to verbally read the ECBI to the child's caregiver and circle the corresponding answers. Only an ID number was written on the ECBI. ECBI forms were then sent by the agency to the researcher.

Treatment as usual day-one workshop. Therapists in the treatment as usual group (TAU) completed an attention control workshop focused on compassion fatigue. No SCIT skills were taught. At the beginning of the training, TAU therapists learned that they were receiving an attention control (compassion fatigue) workshop, and a rationale for the importance of such a workshop was provided. Initial workshop procedures, including an explanation of the research project, research consent forms, and assessment, occurred as previously described for the SCIT therapists. Following assessment, TAU therapists were systematically divided into small groups. Led by WVU staff, a discussion of treatment as usual procedures was conducted. A list of predetermined questions were used to guide discussions. WVU staff recorded therapist's responses 
to TAU questions (e.g., the nature of their job, a typical therapy session, supervision procedures).

See Appendix K for a list of such questions. Following lunch, therapists were presented with a powerpoint presentation on the definition of and warning signs of compassion fatigue. WVU staff facilitated a discussion regarding therapist's thoughts and feelings regarding compassion fatigue. Videos were shown, and the presentation concluded with a discussion of compassion fatigue prevention strategies. A relaxation session was conducted. Therapists played a game to foster positive social relationships, another compassion fatigue prevention strategy. Finally, therapists were presented with toy bags, reflected on the workshop and completed a workshop satisfaction form. TAU therapists were also asked to give their BSC supervisors the ECBI form. Instructions were included to ask BSC's to verbally read the ECBI to the child's caregiver and circle the corresponding answers. Only an ID number was written on the ECBI. ECBI forms were then sent by the agency to the researcher.

SCIT day-two workshop: Parent skill mastery. Seven weeks following the first SCIT workshop, SCIT therapists returned for a second SCIT workshop. Although therapists were encouraged to practice CDI skills individually with child clients after the day-one workshop, the SCIT protocol was not initiated (i.e., skill modeling, practicing, coding, and feedback with parents) until after the day-two workshop. The first 90 minutes of the second SCIT workshop occurred identically to the first SCIT workshop (i.e., introduction, self-report and simulated play assessment). Following the assessment session, a review of CDI, ADI, selective attention, and DPICS skills occurred. SCIT manuals were distributed, and therapists received a bag of SCITappropriate toys to use with their clients (e.g., potato heads, Legos, crayons, paper). Groups of 23 therapists and 2-3 WVU staff practiced CDI and ADI skills to mastery levels. Selective attention practice also occurred. Finally, therapists attempted to reach $80 \%$ coding reliability 
with a trained WVU coder. Following lunch, therapists completed a DPICS quiz. Therapists then returned to their respective groups and role play situations were set up to allow therapists to practice conducting session 3 , the first session in which they were responsible for treatment delivery. Finally, the group reconvened and questions were answered. Finally, therapists reflected on the workshop and completed a workshop satisfaction form. Following this workshop, SCIT therapists were given SCIT manuals and toys so that they could begin using the SCIT protocol with their identified family.

Treatment as usual day-two workshop. The first 90 minutes of the attention control workshop occurred as stated for the previous TAU workshop (i.e., introduction, self-report and simulated play assessment). Following assessment, a Powerpoint presentation of vicarious trauma including a definition and conceptualization occurred. Specifically, the empirical base for exposure was presented, and WVU staff facilitated a discussion among therapists regarding their experiences working with traumatized clients. Agency-level variables facilitating or detracting from therapist's abilities to discuss and process their work occurred. Following lunch, a discussion of prevention strategies to decrease vicarious trauma and improve emotional coping occurred. Videos and a powerpoint presentation illustrated such strategies. Therapists also learned prevention techniques such as progressive muscle relaxation and meditation. A progressive muscle relaxation session was conducted to conclude the workshop. Finally, therapists reflected on the workshop and completed a workshop satisfaction form.

SCIT and treatment as usual post-treatment evaluation and workshop. The daythree workshops for both the SCIT and TAU groups were very similar. Both occurred 7 weeks after the second workshop, lasted for three hours, and included identical assessment procedures (i.e., introduction, self-report measures and simulated play assessment). Following assessment, 
WVU staff collected SCIT therapists' treatment manuals and a discussion of therapists' experiences implementing SCIT occurred. SCIT therapists also completed SCIT evaluation forms (see Appendix F) documenting their thoughts on the impact of SCIT on their client. Finally, therapists completed a workshop satisfaction form.

Following assessment, TAU therapists received a brief powerpoint presentation explaining the purpose and basic skills involved in SCIT. Finally, TAU therapists completed a workshop satisfaction form.

\section{Consultation Calls}

SCIT therapists were required to participate in three consultation calls with WVU staff following the second workshop. Such calls assisted SCIT therapists in conducting procedures with fidelity. Each call lasted between approximately 30 - 60 minutes depending on the number of therapists on the call. See Appendix L for an outline of the consultation call. During the call, SCIT procedures were reviewed. Therapists also discussed current difficulties in the implementation of SCIT with their clients. No client identifying information was disclosed. Following discussion of SCIT clients, DPICS coding practice occurred whereby WVU staff created simple coding questions and therapists coded each phrase using the DPICS.

\section{Missing Data}

Preliminary analyses included an examination of missing data. Missing data for each measure were evaluated and handled according to the specific directions for the given measure or by utilizing appropriate statistical procedures (e.g., mean imputation). If missing data were present for an individual demographic variable, the individual therapist was excluded from analysis of that single variable. For all chi-square analyses with low cell counts (expected values less than five), Fishers exact test was used. 


\section{Results}

\section{Participants}

Therapist demographic information. Demographic information was available for each of the 73 therapists. Sixty-two (84.9\%) of the therapists were female. See Table 2 for a summary of demographic variables for the SCIT and TAU groups combined at the day one workshop. Therapists who dropped out and completed the current study were compared using chi-square and independent samples t-tests on each of the demographic variables at time one. No significant differences were present (all $p$ 's $>.05$; See Table 2 for a list of demographic variables). Analyses were also conducted to determine whether there were significant differences between the SCIT and TAU therapists before treatment. See Table 3 for a summary of chi-square and independent samples t-test analyses for therapists at the day one workshop.

The SCIT group was composed of 36 female therapists and five male therapists while the TAU group was composed of 26 female therapists and six male therapists. A Pearson chi-square test did not indicate significant differences between groups on therapist sex, $X^{2}=.60, p=.52$. Sixty-four therapists $(87.7 \%)$ were Caucasian, four indicated that they were of another race, three (4.1\%) were African American, and two (2.7\%) were of Latino/a/ Hispanic descent. A Pearson chi-square test did not indicate significant differences between groups on number of Caucasian versus non-Caucasian therapists, $X^{2}=.57, p=.49$.

Fifty six therapists (76.71\%) had earned a bachelor's degree. Of these 56 therapists, six (10.9\%) had also pursued some graduate training and one (1.8\%) indicated having pursued another form of education. Additionally, nine (12.33\%) therapists possessed some graduate training and four had an associate's degree. Of these four, three (75\%) therapists had also earned another form of education. Three (4.1\%) had earned a master's degree, and only one (1.4\%) 
reported having earned another form of education. An independent samples $t$ test did not indicate significant differences between groups on therapist years of primary education, $t(53)=-.95, p=$ .35 .

On average, therapists reported having been in their current position for an average of 39.34 months $(S D=50.89)$. Specifically, therapists in the SCIT group had been in their current position for an average of 42.45 months $(S D=58.32)$, while therapists in the TAU group had been in their current position for $35.32(S D=39.87)$ months. An independent samples $t$ test did not indicate significant differences between the SCIT group and the TAU group with regard to months in current position, $t(69)=.58, p=.56$. Many therapists had been working as a therapist for significantly longer than their current position $(M=45.15, S D=57.01)$. Again, an independent samples $t$ test did not indicate significant differences between the SCIT group $(M=$ $46.66, S D=60.94)$ and the TAU group $(M=43.22, S D=52.45)$ with regard to total time as a therapist, $t(71)=.25, p=.80$.

Therapists had an average of 12.49 total child clients during their career $(S D=16.92)$, and an independent samples $t$ test did not indicate significant differences between the SCIT group $(M=11.60, S D=11.68)$ and the TAU group $(M=13.59, S D=21.95)$ with regard to total child clients during their career, $t(70)=-.49, p=.62$. Therapists reported having an average of 3.23 child clients $(S D=1.86)$ currently on their caseload. An independent samples $t$ test did not indicate significant differences between the SCIT group $(M=3.34, S D=1.85)$ and the TAU group $(M=3.09, S D=1.89)$ with regard to number of child clients currently on their caseload, $t$ $(71)=.56, p=.58$

Therapists had been with the client for an average of $8.21(S D=6.79)$ months. An independent samples $t$ test did not indicate significant differences between the SCIT group $(M=$ 
$8.6, S D=7.74)$ and the TAU group $(M=7.75, S D=5.52)$ with regard to total time with client, $t$ $(68)=.52, p=.60$. Most therapists spent an average of 8.35 hours $(S D=5.86)$ per week with their study related client. An independent samples $t$ test did not indicate significant differences between the SCIT group $(M=7.51, S D=5.62)$ and the TAU group $(M=9.42, S D=6.07)$ with regard to the average hours spent per week with the client, $t(71)=-1.39, p=.17$. Prior to study participation, therapist SCIT therapists had spent an average of 8.6 months with their clients while working 7.51 hours per week. Therefore, SCIT clients had received an average of 258.34 hours of previous TSS service, prior to study enrollment. Similarly, TAU therapists had spent an average of 7.75 months and 9.42 hours per week with their client prior to participation. Therefore, TAU clients received an average of 292.02 hours of TSS service prior to the current study.

Therapists had an average of $13.72(S D=18.13)$ hours of previous training in other areas (e.g., ABA, Behavior Modification). An independent samples $t$ test indicated significant differences between the SCIT group $(M=8.70, S D=7.20)$ and the TAU group $(M=19.83, S D=$ 24.74) with regard to other types of therapist training, $t(49)=-2.27, p=.028$. Few therapists possessed previous knowledge of or training in PCIT (7.14\%). A Pearson chi-square test did not indicate significant differences between groups on previous knowledge of or training in PCIT, $X^{2}=.071, p=1.00$ between the SCIT group $(M=1.92, S D=.27)$ and the TAU group $(M=1.94$, $S D=.25)$. On average, therapists attended $2.26(S D=.85)$ workshops. An independent samples $t$ test did not indicate significant differences between the SCIT group $(M=2.15, S D=.82)$ and the TAU group $(M=2.41, S D=.87)$ with regard to number of workshops attended, $t(71)=-1.30, p$ $=.20$. 
Child client demographic information. Demographic information was provided by therapists for 71 child clients. Child clients who dropped out and completed the current study were compared using chi-square and independent samples t-tests on each of the following demographic variables at time one. No significant differences were present. See Table 4 for a complete summary of child demographic variables between groups at the day one workshop. Additional analyses were conducted to determine if significant differences existed between child clients in the SCIT and TAU groups before treatment.

Sixty one (85.9\%) child clients were Caucasian and of those, two (3.28\%) also indicated that they were African American. Additionally, five (7.04\%) therapists indicated that their child clients were of another racial group. Three (4.23\%) clients were Latino/a/Hispanic and two (2.8\%) were of African American descent. A Pearson chi-square test did not indicate significant differences between groups on number of Caucasian versus non-Caucasian clients, $X^{2}=2.92, p=$ .17. See Table 5 for a summary of chi-square and independent samples t-test analyses for child clients at the day one workshop.

On average child clients were 5.44 years old $(S D=1.83)$ at the first assessment. An independent samples $t$ test did not indicate significant differences between the SCIT group ( $M=$ $5.56, S D=2.01)$ and the TAU group $(M=5.28, S D=1.59)$ with regard to child age, $t(71)=$ $.644, p=.521$. On average child clients were male $(n=54,76.1 \%)$. A Pearson chi-square test did not indicate significant differences between the SCIT group and the TAU group on client sex, $X^{2}=2.21, p=.14$.

Child clients possessed a range of diagnoses including: Autism Spectrum Disorder (ASD) ( $n=44)$, Attention Deficit Hyperactivity Disorder (ADHD) $(n=19)$, Oppositional Defiant Disorder (ODD) ( $n=16)$, Conduct Disorder (CD) $(n=3)$, Disruptive Behavior Disorder Not 
Otherwise Specified (DBDNOS) ( $n=18)$, Post-Traumatic Stress Disorder (PTSD) $(n=2)$, Intellectual Disability (ID) $(n=2)$, and another diagnosis $(n=18)$. Specifically, 23 children in the SCIT group and 21 children in the TAU group were reported to have been diagnosed with an Autism Spectrum Disorder and 17 children in the SCIT group and 12 children in the TAU group were reported to have been diagnosed with a disruptive behavior disorder (i.e., ODD, CD, DBDNOS). A Pearson chi-square test did not indicate significant differences between groups in terms of presence of children with an Autism Spectrum Disorder, $X^{2}=.68, p=.41$ or Disruptive Behavior Disorder, $X^{2}=.118, p=.73$.

Clients had been involved in mental health services for an average of 26.59 months $(S D=$ 22.06), and an independent samples $t$ test did not indicate significant differences between the SCIT group $(M=27.41, S D=24.02)$ and the TAU group $(M=25.61, S D=19.90)$ with regard to previous client involvement in mental health services, $t(55)=.31, p=.76$.

Finally, most child clients were not involved in child protective services (CPS) currently (97.3\%), nor in the past (83.6\%). A Pearson chi-square test did not indicate significant differences between groups in terms of current CPS involvement, $X^{2}=.03, p=1.0$ or past CPS involvement, $X^{2}=.70, p=.51$.

Taken together, results revealed that the SCIT and TAU groups were approximately equal on all therapist and child demographic variables. The only difference noted was hours of previous therapist training in which treatment as usual therapists possessed significantly more hours of previous training as compared to SCIT therapists.

SCIT fidelity and dose. On average SCIT therapists mastered 6 (75\%) of the eight possible mastery categories across two workshops (CDI mastery, ADI mastery, $\geq 80 \%$ coding reliability with an advanced coder, and $\geq 80 \%$ correct on a DPICS knowledge quiz). Twenty 
(57.14\%) protocol binders of those SCIT therapists who began SCIT were returned to the researcher. Additional protocols were not returned due to therapist or client drop-out or therapist failure to return the manual. One therapist conducted SCIT with two clients and both binders were returned. The following results were based on such binders. On average, parents of SCIT clients $(n=19)$ completed $19.25(55.74 \%, S D=17.41)$ days of CDI homework and 16.25 $(57.34 \%, S D=15.28)$ days of ADI homework. On average, SCIT therapists implemented 43.39 hours of treatment as usual with SCIT clients. Furthermore, SCIT therapists $(n=19)$ conducted 7.82 hours $(S D=4.53)$ of pure SCIT. Therefore, on average, SCIT was implemented for $16.68 \%$ of total treatment time over the seven week period following the second workshop and prior to the third workshop. When the average number of SCIT sessions was calculated based on therapists completing $\geq 80 \%$ of fidelity criteria per session, therapists completed an average of eight sessions $(S D=4.47)$. When the average number of SCIT sessions was calculated by including any session in which a therapist had noted completing any SCIT procedures, therapists completed an average of 8.45 sessions $(S D=4.11)$. A correlational table comparing workshop two to three parent reported ECBI difference scores to select SCIT therapist fidelity and dose variables (i.e., workshop mastery, time with client, hours per week with client, total SCIT sessions, total SCIT hours, percentage of treatment as SCIT) is presented in Table 6. Dose was not found to be significantly correlated with ECBI difference scores (all $p$ 's $>.05$ ).

Thirteen (65\%) parents of SCIT therapists achieved CDI mastery while 19 (95\%) parents achieved ADI mastery during treatment. Three additional participants (15\%) mastered $80 \%$ of CDI skills within an average of $7.67(1.53)$ sessions while another three participants (15\%) mastered 50\% of CDI skills within an average of 2.67 (1.53) sessions. On average, CDI mastery was achieved within $7.23(S D=3.44)$ SCIT sessions while ADI mastery was achieved within 
$3.47(S D=.84)$ SCIT sessions. Furthermore, on average, children received $62.75(S D=29.38)$ commands throughout ADI, of which compliance was achieved $77 \%$ of the time within three command prompts. Across treatment, four (20\%) children reached the final (restriction of privilege) stage of the ADI sequence in a total of five separate commands (.4\%). See Figure 1 for the median CDI skills (i.e., labeled praise, reflection, behavior description) and don't skills (i.e., questions, commands, negative talk) by session throughout treatment across participants. An evaluation of consult call attendance, including eight of the 13 therapists who completed SCIT ( $\geq$ 8 sessions) indicated that therapists completed an average of 2.75 of the 3 consultation calls.

\section{Primary Analyses}

ECBI parent. See Table 7 for a complete list of parent-reported ECBI scores by assessment for SCIT and TAU clients across all three workshops. See Figure 2 for a graph of parent ECBI mean scores between workshops one, two, and three. A 3 (Time: Workshop 1, Workshop 2, Workshop 3) X 2 (Condition: SCIT, TAU) Analysis of Variance was conducted to examine the impact of study condition and time on parent report of the intensity of child disruptive behavior problems as determined by the ECBI Intensity Scale. All analysis of variance assumptions were met. An interaction of Condition X Time $F(2,19)=1.187, p=.33$ was nonsignificant. However, a main effect of Condition was significant, $F(1,20)=5.374, p=.031$ indicating that significant differences existed for participants between conditions. A main effect of time was not present, $F(2,19)=2.995, p=.074$. Descriptive statistics, $\mathrm{F}$ ratios and $\mathrm{p}$ values are presented in Table 8.

Effect size calculations were conducted using Cohen's $d$ were examined to determine the magnitude of the effect of SCIT over the course of each of the three workshops. Specifically, an effect size of -.93 for the SCIT group indicated a large effect of SCIT, while an effect size of -.01 
for the TAU group indicated no effect of TAU on the ECBI parent scores between workshops one, two, and three (See Table 8). Furthermore, an effect size of -.489 was calculated to compare groups using Lenhard and Lenhard (2016). The average of the day one workshop and two means and standard deviations were used to determine pretest effect sizes. Such calculations indicated a medium effect when the SCIT outcomes were considered relative to the TAU outcomes.

See Table 9 for a complete list of individual parent-reported ECBI scores by assessment for SCIT and TAU clients between workshops two and three. See Figure 3 for a graph of parent ECBI mean scores between workshops two, and three. A second 2 (Time: Workshop 2, Workshop 3) X 2 (Condition: SCIT, TAU) Analysis of Variance was conducted to examine the impact of study condition and time on parent report of the intensity of child disruptive behavior problems as determined by the ECBI Intensity Scale. All analysis of variance assumptions were met. A significant interaction of Condition X Time $F(1,33)=5.135, p=.03$ was present indicating that parent ECBI scores of children in the SCIT group decreased significantly more than parent ECBI scores of children in the TAU group. Post hoc comparisons using the Tukey HSD test indicated that the mean score for the SCIT group at workshop two $(M=159.79, S D=$ 33.95) was significantly different from the mean score for the SCIT group at workshop three ( $M$ $=133.76, S D=30.05), p<.01$ while the mean score for the TAU group at workshop two ( $M=$ 140.94, $S D=36.32$ ) was not significantly different from the mean score for the TAU group at workshop three $(M=134.44, S D=27.55), p>.05$. Furthermore, post hoc comparisons using the Tukey HSD test indicated that the mean score for the SCIT group at workshop two ( $M=159.79$, $S D=33.95)$ was significantly different from the mean score for the TAU group at workshop two $(M=140.94, S D=36.32), p<.05$ while the mean score for the SCIT group at workshop three $(M=133.76, S D=30.05)$ was not significantly different from the mean score for the TAU group 
at workshop three $(M=134.44, S D=27.55), p>.05$. A main effect of Condition was not present, $F(1,33)=.828, p=.369$. However, a main effect of Time was significant, $F(1,33)=$ $14.247, p=.001$ indicating that the average ECBI score increased significantly for the combined group of participants over time (SCIT + TAU). See Figure 4 for a pie chart representing children who improved, worsened, and remained the same on the ECBI between workshop two and workshop three from the parent's perspective. Based on previous literature, a benchmark cutoff of $15 \%$ for each of the three categories was used due to the shortened length and decreased intensity of the intervention (McNeil et al., 1991). Descriptive statistics, F ratios, and p values are presented in Table 10.

Effect size calculations conducted using Cohen's $d$ were examined to determine the magnitude of the effect of SCIT over the course of seven weeks between the time period just prior to and following the implementation of SCIT. Specifically, an effect size of -.812 for the SCIT group indicated a large effect of SCIT, while an effect size of -.202 for the TAU group indicated a small effect of TAU on the ECBI parent scores between workshops two and three (see Table 10). Furthermore, an effect size of -.56 was calculated to compare groups using Lenhard and Lenhard (2016). Such calculations indicated a medium effect when the SCIT outcomes were considered relative to the TAU outcomes.

ECBI therapist. Descriptive statistics for therapist ECBI scores in workshops one, two, and three are presented in Table 8. A 3 (Time: Workshop 1, Workshop 2, Workshop 3) X 2 (Condition: SCIT, TAU) Analysis of Variance was conducted to examine the impact of study condition and time on therapist report of the intensity of child disruptive behavior problems as determined by the ECBI Intensity Scale. All analysis of variance assumptions were met. An interaction of Condition X Time $F(2,34)=2.23, p=.124$ was non-significant, and a main effect 
of condition was not significant, $F(1,35)=.817, p=.372$. A main effect of time was present, $F$ $(2,34)=16.65, p=.000$ indicating that the average ECBI score increased significantly for the combined group of participants over time (SCIT + TAU). See Figure 5 for a graph of therapist ECBI scores between workshops one, two, and three. See Table 11 for a complete list of individual therapist-reported ECBI scores by assessment for SCIT and TAU clients across all three workshops.

Effect size calculations were conducted using Cohen's $d$ and examined to determine the magnitude of the effect of SCIT during the seven weeks just prior to and following the implementation of SCIT. Specifically, an effect size of -.1.003 for the SCIT group indicated a large effect of SCIT, while an effect size of -.588 for the TAU group indicated a medium effect of TAU (See Table 8). Furthermore, an effect size of -.537 was calculated to compare groups using Lenhard and Lenhard (2016). The average of the day one workshop and day two workshop means and standard deviations was used to determine pretest effect sizes. Calculations indicated a medium effect when the SCIT and TAU interventions were compared following the conclusion of SCIT.

Descriptive statistics are presented in Table 10. A second 2 (Time: Workshop 2, Workshop 3) X 2 (Condition: SCIT, TAU) Analysis of Variance was conducted to examine the impact of study condition and time on therapist report of the intensity of child disruptive behavior problems as determined by the ECBI Intensity Scale. All analysis of variance assumptions were met. An interaction of Condition X Time $F(1,37)=3.69, p=.062$ was nonsignificant. However, a main effect of Condition was significant, $F(1,37)=1.360, p=.251$ indicating that significant differences existed for participants between conditions. A main effect of time was also present, $F(1,37)=26.852, p=.000$ indicating that the average ECBI score 
increased significantly for the combined group of participants over time (SCIT + TAU). See Figure 6 for a graph of children who improved, worsened, and remained the same on the ECBI between workshop two and workshop three from the therapist's perspective. See Figure 7 for a graph of children who improved, worsened, and remained the same on the ECBI between the day one workshop and workshop three from the therapist's perspective. Based on previous literature, a benchmark cutoff of $15 \%$ for each of the three categories was used due to the shortened length and decreased intensity of the intervention (McNeil et al., 1991). See Figure 8 for a graph of therapist ECBI scores between workshops two and three. See Table 12 for a complete list of therapist-reported ECBI scores by assessment for SCIT and TAU clients between workshops two and three.

Effect size calculations were conducted using Cohen's $d$ and examined to determine the magnitude of the effect of SCIT over the course of seven weeks during the seven weeks just prior to and following the implementation of SCIT. Specifically, an effect size of -1.09 for the SCIT group indicated a large effect of SCIT, while an effect size of -.527 for the TAU group indicated a medium effect of TAU (See Table 10). Furthermore, an effect size of -.67 indicated a medium effect when the SCIT and TAU interventions were compared following the conclusion of SCIT.

Comparison of parent and therapist ECBI difference scores. A Pearson correlation was conducted to determine the relationship between parent and therapist ECBI difference scores within the SCIT and TAU groups. A medium correlation between parent and therapist ECBI percentage change was present in the SCIT group $(r=.51, p=.06)$ as compared to a small correlation between parent and therapist ECBI percentage change in the TAU group $(r=.10, p=$ .7). Using the Fisher r-to-z transformation (Lowry, 2001- 2015), correlations were compared and 
differences were not statistically significant $(z=1.15, p=.13)$. However, these analyses involved low sample sizes of 14 (SCIT group) and 17 (TAU group).

\section{Feasibility Questions}

Cost. See Table 13 for a complete cost analysis of anticipated SCIT implementation expenses within community-based settings based on the cost of SCIT training in the current study. Costs are calculated in terms of eight therapists (four therapist-supervisor pairs) attending four, full-day training workshops. Specifically, SCIT manuals cost approximately $\$ 128.64$ while a set of ECBI measures (conducted prior to, in the middle of, and following treatment) cost \$21.60. Together, mastery stars, name tags, DPICS quizzes, pencils, laminated sheets, and certificates cost $\$ 56.64$ per training group. Additionally, food supplied at each workshop was budgeted at $\$ 200$ ( $\$ 800$ over four workshops). A set of SCIT toys cost approximately $\$ 34.56$ ( $\$ 138.24$ for four pairs). Therefore, the total cost of SCIT training materials was $\$ 1,145$ per group of eight therapists ( $\$ 143.13$ per therapist).

The relatively low cost of SCIT implementation in the current study was heavily influenced by the leadership of graduate and undergraduate psychology students as part of dissertation, master's thesis, and undergraduate thesis milestone projects. Therefore, no costs associated with trainer salaries nor cost of facilities were present. Given the unique circumstance of such implementation, analysis of cost in the current study was based on anticipated, projected costs of implementation in community-based settings.

Attrition Status. Client study-related attrition resembled therapist attrition closely due to interconnected therapist-client dyads. Only slight differences between groups existed. Therapist and client attrition status were determined by assigning a quantitative value to one of five potential descriptive codes per therapist and client. SCIT therapist and client attrition codes were 
as follows: (1) completed study procedures (therapist: $n=20,45.45 \%$; client: $n=18,40.91 \%$ )

(2) stopped responding to calls for unknown reason (therapist: $n=6,13.64 \%$; client: $n=6$, 13.64\%), (3) withdrew from TSS position (therapist: $n=9,20.45 \%$ ) or switched therapist (client: $n=5,11.36 \%)$, (4) lost study-related client, did not get new client (therapist: $n=3,6.82 \%$ ), and (5) never began study procedures with a client (therapist and client: $n=6,13.64 \%$ ). TAU therapist attrition codes were as follows: (1) completed study procedures (therapist: $n=22$, 68.75\%; client: $n=22,68.75 \%$ ), (2) stopped responding to calls for unknown reason (therapist and client: $n=2,6.25 \%$ ), (3) withdrew from TSS position (therapist: $n=7,21.88 \%$ ) or switched therapists (client: $n=1,3.13 \%$ ), (4) lost study-related client, did not get new client (therapist: $n=$ $1,3.13 \%$ ), and (5) never began study procedures with a client (therapist and client: $n=0,0 \%$ ).

A Pearson chi-square analysis revealed that therapists $(N=73)$ were not significantly more likely to drop out of the SCIT condition as compared to the TAU condition, $X^{2}=3.66, p=$ .056 , but there was a trend towards higher attrition in the SCIT group. A second Pearson chisquare analysis revealed that clients $(N=73)$ were significantly more likely to drop out of the SCIT condition as compared to the TAU condition, $X^{2}=5.38, p=.02$.

Given the discrepancy between SCIT and TAU therapists and clients regarding the percentage of therapists unable to complete study procedures due to the lack of an appropriate client (SCIT: $n=6,13.64 \%$; TAU: $n=0,0 \%$ ) and percentage of clients who never participated in study procedures (SCIT: $n=6,13.64$; TAU: $n=0.0 \%$ ), an additional Pearson chi square analysis was conducted following the removal of the 'never had a client' therapist category and 'never was a client' client category. All additional codes remained the same. When considering only those therapists who had an appropriate client $(n=67)$ and clients who initially participated in study procedures, no significant differences in attrition were evident for therapists or clients 
assigned to the SCIT condition versus the TAU condition, $X^{2}=1.47, p=.225$. See Table 14 for a description of individual SCIT and TAU therapist attrition throughout workshops one, two, and three.

Therapist workshop feedback. Following each workshop, SCIT and TAU therapists provided an evaluation of workshop quality. Each SCIT therapist's $(n=39)$ and TAU therapist's $(n=32)$ quantitative scores were averaged across workshops attended. An independent samples $t$ test indicated significant differences between the SCIT group $(M=34.42, S D=1.65)$ and the TAU group $(M=31.59, S D=2.33)$ on therapist workshop satisfaction, $t(69)=5.98, p<.01$. Therapists also provided feedback regarding the most preferred aspect of the workshop and suggestions for improvements.

SCIT workshop feedback. SCIT therapists' positive reactions to the workshops fell into six overarching categories including workshop structure, learning new skills and information, workshop format, opportunities for skill practice, instructor qualities, and workshop discussions. Overall, therapists appreciated the hands-on, interactive, practice-oriented activities. Small group break-out sessions to practice skills were commonly cited as a positive aspect of the SCIT workshops. Such small group practice and discussions facilitated learning and sustained interest in workshop material. In addition to a positive impact upon therapist confidence, therapists also commented upon the applicability of the knowledge gained for use with current and future clients. SCIT therapists noted their high level of comfort within the workshops and the ease with which instructors answered participants' questions. SCIT therapists also noted the organized nature of the workshop. The knowledgeable, supportive, friendly, and enthusiastic nature of the instructors were commonly noted by SCIT therapists. Finally, SCIT therapists appreciated the opportunity to interact with other TSS regarding their work with challenging child clients. 
Suggestions for change focused on improvements in workshop structure, setting, and workshop content. Some therapists commented upon the lack of breaks throughout the training day. They indicated a preference for a longer training day or multiple training days with more built-in breaks. Therapists also commented upon the facilities including the hard-back chairs and travel distance necessary for workshop attendance. Finally, therapists indicated that workshop material was presented relatively quickly and a preference for additional instruction on working with parents was noted. See Table 15 for a summary of themes and representative responses regarding strengths of the SCIT workshops and suggestions for improvement.

TAU workshop feedback. TAU therapists' positive reactions to the workshops fell into six overarching categories including the workshop structure, compassion fatigue material, agency comments, discussion, instructor qualities, and SCIT review. Specifically, therapists appreciated the fun, hands-on activities, interactive and collaborative nature of the workshop, in addition to the open atmosphere, appropriate activities and materials, and professional presentation. Additionally, therapists noted their appreciation of the compassion fatigue material and specifically enjoyed learning about relaxation skills, meditation, muscle relaxation, tactics to prevent burnout, and compassion fatigue. Therapists appreciated the opportunity to present ideas to decrease turnover within the agency. Therapists also noted an appreciation for agency quality improvement. Additionally, therapists commonly cited the group discussion, games, and opportunity to speak with fellow TSS about their job and experiences as strengths of the workshops. They appreciated the opportunity to express concerns, voice opinions, and hear advice from TSS from offices beyond their own. Positive instructor qualities including their welcoming, non-judgmental, and friendly nature. Finally, TSS positively commented on the opportunity to learn about SCIT during the final TAU workshop. 
Suggestions for change focused on workshop content, workshop quality, instructor qualities, training opportunities, and assessment utilized. Specifically, TAU therapists indicated their desire to learn more about SCIT. Therapists also commented on the long length of the workshop, desire for more comfortable chairs, and healthier snacks. Next, therapists commented upon the need for instructors to gain a clearer understanding of the TSS position in order to discuss realistic and accurate scenarios throughout the training. Therapists indicated confusion regarding the purpose and focus of the training, expecting a greater focus on client-based skills. Some therapists suggested that the presenters have greater communication with the agency regarding the training. Finally, TAU therapists commented upon the use of the role-play assessments and desire for preparation prior to the assessment. See Table 16 for a summary of themes and representative responses regarding strengths of the TAU workshops and suggestions for improvement.

Therapist sense of competence. See Table 17 for results of the SSOC between groups across each of the three time points. See Figure 9 for a graph of therapist SSOC scores between workshops one, two, and three. A 3 (Time: Workshop 1, Workshop 2, Workshop 3) X 2 (Condition: SCIT, TAU) Analysis of Variance was conducted to examine the impact of study condition and time on therapist report of therapist competence as determined by the SSOC. All analysis of variance assumptions were met. An interaction of Condition X Time $F(2,37)=6.12$, $p=.01$ was significant. A main effect of Time was significant, $F(2,37)=12.78, p=.00$ indicating that the average SSOC score increased significantly for the combined group of participants over time (SCIT + TAU). However, a main effect of condition was not present, $F(1$, $38)=2.8, p=.10$. Post hoc comparisons using the Tukey HSD test indicated that the mean score for the SCIT group at workshop one $(M=72.16 .79, S D=10.42)$ was not significantly different 
from the mean score for the SCIT group at workshop two $(M=75.52, S D=9.82), p>.05$ but the mean score for the SCIT group at workshop two $(M=75.52, S D=9.82)$ was significantly different from the mean score for the SCIT group at workshop three $(M=81.11, S D=11.03), p$ $<.05$. Similarly, the mean score for the SCIT group at workshop one $(M=72.16 .79, S D=10.42)$ was significantly different from the mean score for the SCIT group at workshop three ( $M=$ 81.11, $S D=11.03), p<.01$. Post hoc comparisons using the Tukey HSD test also indicated that the mean score for the TAU group at workshop one $(M=71.81, S D=9.91)$ was not significantly different from the mean score for the TAU group at workshop two $(M=70.92, S D=7.29), p>$ .05 and the mean score for the TAU group at workshop two $(M=70.92, S D=7.29)$ was not significantly different from the mean score for the TAU group at workshop three $(M=72.90, S D$ $=9.55), p>.05$. Similarly, the mean score for the TAU group at workshop one $(M=71.81, S D=$ 9.91) was not significantly different from the mean score for the TAU group at workshop three ( $M=72.90, S D=9.55), p>.05$. Additionally, post hoc comparisons using the Tukey HSD test indicated that the mean score for the SCIT group at workshop one $(M=72.16 .79, S D=10.42)$ was not significantly different from the mean score for the TAU group at workshop one ( $M=$ $71.81, S D=9.91), p>.05$ but the mean score for the SCIT group at workshop three $(M=81.11$, $S D=11.03$ ), was significantly different from the mean score for the TAU group at workshop three $(M=72.90, S D=9.55), p<.01$.

Effect size calculations were conducted using Cohen's $d$ and examined to determine the magnitude sense of competence changes for the SCIT versus TAU therapists over the seven week time period. The average of the day one workshop and two means and standard deviations were used to determine pretest effect sizes. Specifically, an effect size of .686 for the SCIT group indicated a medium effect of SCIT, while an effect size of .207 for the TAU group indicated a 
small effect of TAU. Furthermore, an effect size of .571 was calculated between groups using Lenhard and Lenhard (2016). Such calculations indicated a medium effect when the SCIT and TAU interventions were compared before and after the workshops. Such results indicate that therapists in the SCIT group had significantly greater increases in SSOC scores following SCIT compared to TAU group SSOC changes over the same time period.

Treatment as usual description. Focus group discussions with TAU therapists explored a variety of noteworthy qualitative themes related to therapist retention and satisfaction in the TSS position. TAU therapists noted that they receive BHRS client referrals based on TSS availability. Upon referral, the TSS is provided with a child's age, location, and hours prescribed to the child. TAU therapists reported varying lengths of time with consumers prior to graduation. Variables such as insurance restrictions, client progress, and family choice may significantly influence a client's length of BHRS services. While some therapists indicated that a client may be in services for as little as three months, others noted that services often continue for years. Weekly, therapists reported spending between 1.5-15 hours per week with the client. Therapists indicated that typical treatment goals included increasing compliance, communication, functional living skills, sustaining attention, coping skills, decreasing aggression, social skills, and increasing the client's safety. In an effort to accomplish such goals, TSS reported engaging in a variety of activities based in the client's home and community environments. Some TSS indicated that activities are often classified as either preferred and non-preferred and sequenced with the use of a visual schedule during BHRS sessions. Guided by the treatment plan, games, play with siblings, eating, natural environment learning, fine motor activities, and free play activities are used as a platform for fulfilling such goals. SCIT therapists qualitatively indicated additional treatment as usual activities completed during the remainder of the SCIT session. 
Activities fell under the following eight overarching categories: social skills (e.g., turn taking, sharing toys, reciprocal interactions with siblings), academic skills (e.g., reading, journal writing, letter recognition, color matching, numbers, homework, ABC's, food identification, matching shapes, picture identification), self-help/care skills (e.g., eating dinner, potty time, cleaning room), compliance/transitioning (e.g., following directives, transitioning without negative behaviors), communication skills (e.g., using words [to express him/herself]), games (e.g., game of "Sorry", puzzles, large muscle obstacle course, story, play doh), emotional expression/impulse control, and programming (e.g., questions and compliance, identifying stimuli).

At times, the TSS, child, and his or her parent(s) undertake sessions in public places such as the zoo, restaurants, the science center, library, during extra-curricular activities, and grocery stores. Methods such as intensive teaching, sensory bins, prompting, choices, engagement in non-preferred tasks, and rewards for task completion are used to accomplish goals within session. Progress is measured using session-by-session data sheets to track goal behaviors (e.g., compliance) depending upon the individual needs of the child. In order to accomplish a goal, a client must exceed the goal $80 \%$ of the time. Data sheets and the accompanying session note must be submitted within 24 hours of the session. Formal re-evaluations of client progress are conducted every six months to determine alterations in treatment hours. Finally, therapists commented upon the conclusion of services indicating that termination may occur for a variety of reasons including goal fulfillment, parent termination of services, family move, insurance changes, lack of family engagement, family drop out, or when a team determines that services are no longer necessary. A few therapists noted that throughout their one to two years as a TSS and work with six to eight clients, they had had one or two families formally graduate from services. 
Children may also receive services in the school environment by the same home-based TSS or by a separate TSS. Within the school setting, the TSS remains close to the child and only engages with the child if he or she is off-task, non-compliant, or engages in misbehavior. Additionally, the TSS commented on behavior management systems such as flipping colorcoded cards, sending notes home to parents, and participating in meetings with the principal in an attempt to manage child misbehavior. See Table 18 for a summary of qualitative themes present in discussions with TAU therapists.

SCIT implementation feedback. SCIT therapists provided feedback at the third SCIT workshop regarding their experiences providing SCIT to child clients. Specifically, therapists indicated desired outcomes following SCIT implementation (e.g., appropriate play skills and improved social interaction, increased compliance, decreased aggression). Therapists also commented upon client changes (e.g., increased eye contact, requests to play with parents, increased compliance, increased confidence, and improved expression of needs and wants), parent changes (e.g., caregivers interact with client more positively and effectively, caregiver is so much more involved, caregivers more aware of positive behaviors), and therapist changes (e.g., skills in implementing and in overall interactions, realized the parent-child relationship is important, therapist more aware of negative talk, using PRIDE skills with everyone) following SCIT. Finally, therapists noted barriers to SCIT implementation (e.g., child/parent health issues, getting BSC on board, family canceling BHRS services without notice) as well as suggestions for improvement in the SCIT program (e.g., different way to track homework, toys for older kids, more training for BSC). Results are presented in Table 19. 


\section{Discussion}

\section{Overview of the Present Study}

The current study examined the implementation of SCIT, a novel adaptation of ParentChild Interaction Therapy for children between the ages of two and nine years of age with disruptive behaviors involved in the wraparound system of care. Training in either SCIT skills or an attention control condition occurred during the course of two, full-day workshops followed by a half-day, post-treatment evaluation. Bachelors-level therapists implemented SCIT in the context of in-home wraparound service hours for approximately seven weeks following the second SCIT workshop. Attention control therapists received no SCIT skills and continued to implement treatment as usual. A number of notable conclusions can be drawn from the current study, particularly when findings are considered in the context of therapists, parents, and child client's previous experiences in BHRS services.

First, SCIT therapists received 12 continuing education hours of didactic and hands-on training in the current intervention. Although therapists reported an average of 13.72 hours of previous training, high levels of missing data indicate that state-dictated requirements for TSS training hours may provide a more accurate perspective of therapist hours of previous training. Specifically, therapists had been in their current position for an average of 34.34 months ( 2.86 years) and likely received approximately 76.2 hours of training during that time (Community Behavioral Health, 2014). Therefore, the current SCIT training accounted for only $7 \%$ of therapists' overall continuing education training.

Next, prior to study participation, SCIT therapists had delivered services to their clients for an average of 8.6 months and 7.51 hours per week. Therefore, SCIT clients had received an average of 258 previous hours of TSS service, costing approximately $\$ 7,750$ per client (Stokes, 
2014). SCIT therapists implemented an average of 7.82 hours of SCIT in the context of TAU over the course of seven weeks between the second and third workshops, costing approximately \$234 per client (Stokes, 2014). Finally, a number of agency-level, contextual factors may have negatively contributed to the possible impact of the current intervention. First, families' extended involvement in BHRS prior to SCIT caused a notable shift in the structure and focus of treatment sessions from a primarily therapist-child dyadic interaction to a therapist-child-parent triadic interaction. Additional demands were also placed upon parents who were asked to practice SCIT skills outside of sessions with their child. Second, a lack of agency readiness for SCIT implementation caused initial SCIT therapists to pause SCIT services after workshop one in order to train supervisors in the treatment. Supervisors received minimal (one day) training in preparation for SCIT implementation. Third, SCIT represents a low-level intervention for child behavior problems. Unlike PCIT, time-out for non-compliance (a hallmark feature of effective child treatment programs, Kaminski et al., 2008), was not included in SCIT due to the risks involved in requiring bachelors-level therapists to complete this procedure with aggressive children in the uncontrolled home environment. Instead, a restriction of privilege procedure was included that enabled children to escape from the original command following repeated noncompliance and the provision of the privilege restriction. Taken together, results indicate that the current implementation of SCIT included low levels of training, few hours of intervention, and a minimally intensive behavioral procedure relative to previous therapist training and client intervention experiences in BHRS. Additionally, a number of agency-level and policy-based contextual factors may have negatively contributed to the most effective delivery and evaluation of the intervention in the current study. 
In light of such limitations, evidence of change across each of the five dependent variables was present. Results indicated that SCIT significantly decreased the intensity of children's disruptive behavior, based on parent report following full treatment implementation. Such results are in line with previous research indicating improved behavior in community-based environments (McNeil \& Hembree-Kigin, 2010). Therapist's evaluation of child behavior problems indicated a trend toward significance. Effect sizes for both groups ranged from medium to large, indicating more powerful treatment effects for children receiving SCIT as compared to those in the treatment as usual condition. Feasibility analyses examined attrition, cost, and acceptability of SCIT as an intervention within the wraparound system of care. Results indicated that policy-level and agency readiness variables may have contributed to attrition rates. However, following the removal of therapists who were not able to begin SCIT due to the lack of an appropriate client, no significant differences in attrition between groups were present. The cost of SCIT workshop materials for future, community-based implementations of SCIT for eight therapists was approximately $\$ 1,145$ (i.e., $\$ 143.13$ per therapist). Qualitative discussions with TAU therapists, workshop satisfaction forms, and SCIT feedback surveys revealed that SCIT therapists were more satisfied with workshops as compared to TAU therapists. SCIT therapists also demonstrated significantly higher levels of overall competency following training as compared to TAU therapists. Furthermore, when asked about the impact of SCIT upon their clients, SCIT therapists noted a range of positive emotional, behavioral, and socially-based changes as well as notable improvements in the quality of the parent-child relationship. Finally, discussions with treatment as usual therapists revealed a range of client-based and agency-based limitations which shed light upon the high level of therapist turnover and lack of client progress. 
Taken together, results indicate that SCIT may be a feasible, effective intervention when delivered by bachelors-level therapists in the context of in-home wraparound services.

Although promising, the positive results should be considered preliminary given two major methodological concerns present in the data. Primarily, a lack of participant randomization to condition may have contributed to significantly higher ECBI scores for children in the SCIT group as compared to children in the TAU group at pretreatment. Therefore, it is possible that regression to the mean may have contributed to changes in ECBI scores over time between groups. Additionally, high levels of therapist (SCIT: 54\%, TAU: 31\%) and client (SCIT: 59\%, TAU: 31\%) attrition were present across both SCIT and TAU groups. Although no demographic differences between therapist and client completers versus dropouts within either condition were present, such high levels of attrition resulted in a small sample and low power, potentially increasing Type II error and decreasing the chance that effects could be detected if present. Replication of the current results using stronger methodology in future investigations must occur before definitive conclusions can be reached.

\section{Child Behavior Problems}

Therapist ECBI. The intensity of child disruptive behavior was examined from the perspective of the child's therapist. Significant pre to post differences between conditions were not present from the therapist's perspective when scores were examined across all three workshops. Instead, therapists in both groups reported improved client behavior. However, when child behavior problems were examined only between the second and third workshops (when SCIT was fully implemented), results approached significance between groups $(p=.06)$.

Specifically, average child clients ECBI scores decreased from the $96^{\text {th }}$ to the $79^{\text {th }}$ percentile in the SCIT group as compared to the $89^{\text {th }}$ to the $79^{\text {th }}$ percentile in the TAU group. Effect size 
calculations indicated a medium effect of SCIT as compared to treatment as usual. When examined individually by group, a large effect of SCIT was present across all three time points as compared to a medium effect of TAU from the therapist's perspective.

Although therapists began utilizing SCIT skills individually with the child following the first workshop, no formal implementation of the parent skills training component of the program occurred until after the second workshop. Therefore, treatment as usual services continued with SCIT clients during this time. It is likely that SCIT skills were utilized inconsistently and with low frequency when interacting with the child, resulting in minimal changes between the first and second workshop between groups from the therapist's perspective. The systematic implementation of SCIT services occurred only following the conclusion of the second workshop. At this time, the primary focus on SCIT services shifted from a treatment as usualbased, individual therapy model to a PCIT-based treatment program whereby therapists taught, modeled, and provided feedback to parents during play-based interactions with the child client. SCIT's intensive transfer of skill approach facilitated parental involvement in the previously dyadic therapist-child interaction. Parents spent more time with the child during treatment sessions while they learned and practiced evidence-based, behavior management strategies in real time. Therefore, the impact of such parental involvement on child behavior appeared to affect therapist perceptions of child behavior change in the SCIT group as compared to the TAU group indicating the influence of parental involvement on changes in children's disruptive behavior.

Parent ECBI. The intensity of child disruptive behavior was examined from the perspective of the child's parent. Significant differences between conditions were not present from the parent's perspective when scores were examined across each of the three workshops. 
However, when child behavior problems were examined between the second and third workshops (i.e., after SCIT was implemented with the parents), results indicated significant differences between conditions. Specifically, child clients ECBI scores decreased from the $97^{\text {th }}$ to the $87^{\text {th }}$ percentile in the SCIT group as compared to a decrease from the $91^{\text {st }}$ to $87^{\text {th }}$ percentile in the TAU group. Effect size calculations indicated a medium effect of SCIT as compared to treatment as usual. When the SCIT group was examined individually, a large effect of SCIT was present from workshop two (when SCIT was implemented) to workshop three. No effect of TAU was present when measured across all three workshops and a small effect was present when measured between the second and third workshops. When interpreting these effect sizes, however, it must be noted that the SCIT group parents reported more behavior problems at pretreatment providing greater opportunity for large effects to be achieved in SCIT than in the TAU group.

The finding of larger parent report of behavioral improvements in SCIT versus TAU appeared in line with expectations based on SCIT implementation protocol. In particular, the pattern of SCIT group change (no improvements until after second workshop when parents were included in treatment) was hypothesized. Parents were not expected to notice changes in child behavior in the time between the first and second workshop due to 1) the low intensity of SCIT intervention skills likely utilized between the therapist and child, and 2) the absence of changes in parent involvement prior to the day one workshop and between workshops one and two.

The transfer of skill component appeared to be related to parent report of decreased child behavior problems. Parents may have perceived such improvements in child behavior problems due to a number of key factors. It is likely that parents gained empirically-based child management skills resulting in increased positivity in the parent-child interaction and the 
consistent use of effective behavior management strategies to increase child compliance and decrease aggression. Such results mirror previous findings consistently noted in PCIT research studies (McNeil \& Hembree-Kigin, 2010). Following the transfer of skill component of SCIT, it is likely that bi-directional benefits between the child client and his or her parent occurred. PCIT research demonstrated that children display respect for parent's authority following consistently positive interactions utilizing predictable discipline strategies (McNeil \& Hembree-Kigin, 2010). Similarly, PCIT studies suggest that parents' stress levels decrease and parents' sense of confidence increases, as caregivers are empowered to be the primary mechanism of change in their child's treatment (Eisenstadt, Eyberg, McNeil, Newcomb, \& Funderburk, 1993; Eyberg et al., 2001). Conversely, parents of children in the TAU group were likely to have remained inconsistently involved during this time, resulting in little additional improvement in child behavior from their perspective.

Finally, it is important to note the critical importance of the parent's perspective on child behavior problems given the frequency with which parents view children across a variety of situations over time. As a result, parent report may represent a more valid source of information regarding child behavior change outside of the therapy session, when compared to therapist report. Ultimately, caregivers are the consumers of mental health interventions for their children, such that their positive perspective on child behavior change and satisfaction with services are integral to effective outcomes.

Relationship between therapist and parent reports. Parent report of percent change was compared to therapist report of ECBI change (between the second and third workshops) for therapists in the SCIT versus TAU groups. Although statistically significant differences between correlations were not present $(z=-1.145, p=.126)$, the correlation between SCIT parents and 
therapists indicated a medium correlation $(r=.51)$ as compared to a small correlation between TAU parents and therapists $(r=.10)$. Such differences may have occurred as a function of the intensive parent involvement in SCIT treatment following workshop two, thereby enabling SCIT therapists and parents to recognize similar degrees of change in the child, potentially increasing the validity of SCIT therapists' report of child behavior problems. It is possible that small sample sizes and low power may have increased the chances of Type II error, thereby masking the presence of effects that may otherwise exist. Finally, qualitative analysis indicated that TAU therapists spent more individual time with the child. Therefore, TAU therapists' report of child behavior may be skewed toward their perceptions of the child during such individual interactions. SCIT therapists, however, engaged both the parent and child in treatment sessions. Therefore, SCIT therapists' report of child behavior may be in greater alignment with the parent given their perspective of parent-child interactions. Ultimately, the parent's perspective of the intensity of the child's behavior reflects the most valid and influential account of such difficulties in the child's life given the time spent with the child across a variety of situations.

\section{Feasibility Analyses}

Cost of SCIT implementation. Costs of SCIT implementation in the current study are based on anticipated expenses in a community-based mental health setting. The cost of materials necessary for SCIT training (e.g., manuals, handouts, toys, food) were calculated and totaled $\$ 1,145.12$ per group of 8 therapists (four therapist-supervisor dyads) trained ( $\$ 143.13$ per trainee). It should be noted, however, that such cost analysis does not include trainer salaries, therapist salaries, therapist and trainer travel-related costs, and the cost of space utilization given that such costs vary based on location and agency. 
Given previous estimates indicating that TSS services typically cost $\$ 30$ per hour of service (Stokes, 2014), it is estimated that SCIT services alone cost approximately $\$ 234.60$ per client over the seven weeks of treatment in between the second and third workshops. Notably, SCIT therapists had worked with their clients for an average of 8.6 months and 7.51 hours per week. Therefore, prior to the current study, SCIT clients received an average of 258.34 hours of previous TSS service, costing approximately $\$ 7,750.32$ per client. Additionally, TAU therapists had worked with their clients for an average of 7.75 months and 9.42 hours per week. Therefore, prior to the current study, TAU clients received an average of 292.02 hours of TSS service, costing approximately $\$ 8,760.60$ per client. Taken together, such results indicate that SCIT may be a cost-effective form of training and intervention for children with behavior problems in the context of in-home, community wraparound services as compared to BHRS services as usual.

\section{Attrition}

Therapist attrition. Therapist study-related attrition between groups was examined to better understand the feasibility of SCIT implementation in the context of in-home wraparound services. High attrition rates posed a significant limitation to the representativeness of groups and generalizability of findings, thereby limiting sample size and decreasing the power of results. Less than half of initially enrolled (45.45\%) SCIT therapists completed all study procedures. The majority of therapist study attrition occurred as a result of the following: (a) therapist withdrawal from the TSS position (20.45\%), (b) therapists who never began study procedures with a client (13.64\%), (c) therapists who stopped responding to study related calls (13.64\%), and finally, (d) therapists who lost their study-related client and were not assigned a new client who met study criteria (6.82\%). A higher percentage of TAU therapists as compared to SCIT therapists were retained in the current study $(68.75 \%)$. Similar to SCIT therapists, the primary reason for study- 
related attrition was due to withdrawal from the TSS position (21.88\%), followed by TAU therapists ceasing to respond to study-related calls $(6.25 \%)$, and finally TAU therapists who lost their study-related client and were not assigned a new client who met study criteria (3.13\%). No TAU therapists were unable to complete study procedures due to the lack of an appropriate client. Analyses indicated no differences between SCIT and TAU therapists regarding the likelihood of attrition.

It is notable to address therapist turnover as a consistent concern in both SCIT and TAU groups. In addition to the aforementioned quantitative analysis, qualitative discussions with TAU therapists indicated a number of agency-based and policy-based factors likely related to high therapist attrition rates from the TSS position. Many therapists indicated that the TSS position is often used as a stepping stone to gain experience working with children and families prior to entrance into higher education or higher-level positions. TSS positions are characterized by minimal pay and high levels of job stress. Effective TSS, who display stability, reliability, and competence, are often recruited into higher level positions within the agency or secure similar jobs with higher pay and greater benefits.

Client attrition. Client study-related attrition between groups was also examined to better understand the feasibility of SCIT implementation in the context of in-home wraparound services. Less than half of initially enrolled (40.91\%) SCIT clients completed all study procedures. The majority of client study attrition occurred as a result of therapist withdrawal from the TSS position (20.45\%), followed by therapists who never began study procedures with a client (13.64\%), and therapists who stopped responding to study-related calls (13.64\%) thereby leading to client attrition from the study. Some SCIT clients switched therapists (11.36\%), and $13.64 \%$ of SCIT therapists' identified clients failed to meet criteria or participate in the current 
study. A higher percentage of TAU clients as compared to SCIT clients were retained in the current study (68.75\%). Similar to SCIT therapists, the primary reason for TAU client studyrelated attrition was due to therapist withdrawal from the TSS position (21.88\%), followed by TAU therapists ceasing to respond to study-related calls (6.25\%), and TAU clients who switched therapists $(3.13 \%)$. No TAU clients failed to meet criteria or were unable to participate in the current study.

Descriptive analyses indicated that all clients who never participated in study procedures were clients of SCIT therapists. Additionally, such limitations only occurred during the first half (first two workshops) of SCIT. All clients assigned to SCIT and TAU therapists and eligible to take part in the current investigation during the second half of the study participated in study procedures. Such results indicate the lack of agency preparation for SCIT implementation during the initiation of study procedures. Logistical barriers, unclear communication, and the loss of anticipated study-related clients prior to the first SCIT workshop were the primary reasons for the lack of anticipated SCIT clients' participation in the SCIT program. For example, shortly prior to workshop arrival, a few SCIT therapists indicated that they were only assigned to school hours with the target client and thereby could not implement SCIT in the home setting with such clients. Other SCIT therapists indicated that they were no longer assigned to the anticipated case (e.g., changes in client services prior to workshop participation). At times, such therapists anticipated receiving a new eligible client in the near future and attended the workshop in preparation for the new, anticipated client. Additionally, at this time, the researcher was primarily in charge of study recruitment. Therapists were contacted individually and clients were screened based on therapist report of client eligibility characteristics. Conversely, during the second half of study procedures, agency preparation and communication with the research team 
improved. Additionally, agency assignment of therapists with eligible clients to condition occurred. The influence of such agency-level involvement clearly facilitated assignment of therapists with appropriate clients to conditions.

Similar to therapist withdrawal, client withdrawal from BHRS services significantly contributed to attrition from the current study. Although the particular reasons for such attrition were often unknown to the researcher, it is hypothesized that the consumer-based model, whereby a client's parents maintain primary decision-making power regarding the type and provider of services (e.g., agency provider as well as individual therapist) may have contributed to client attrition. Although the role of the client's family in treatment is critically important to treatment engagement, the unique position of power provided to the parent regarding treatment implementation decisions may also serve to contribute to client-related treatment attrition. Similar to therapists, SCIT therapists reported that SCIT facilitated greater communication between the therapist and parent while also building respect for the specialized, effective skills provided by the therapist to the parent. Thereby, a larger-scale SCIT implementation may reveal a positive impact on premature client-related attrition as parents experience greater clinical success and view the therapist as an intricate part of their child's progress in services.

\section{Acceptability}

SCIT workshop feedback. SCIT therapists provided feedback of SCIT workshops following each successive training. Overwhelmingly, therapist feedback was positive indicating high levels of satisfaction and acceptability of the training experience. Additionally, quantitative results indicated that SCIT therapists were significantly more satisfied with the SCIT workshop as compared to TAU therapists regarding the compassion fatigue workshop. Specifically, SCIT therapists particularly enjoyed the interactive, hands on practice provided throughout the 
workshop noting that such experiences facilitated deep learning and skill acquisition. Therapists commented on the intensive skill practice and resulting positive effect on their confidence level in their use. Therapists felt as though all questions were answered. Therapists noted that they expected acquired skills to be useful with a variety of children and families across a multitude of situations. SCIT therapists also felt supported as they were provided with the opportunity to discuss TSS-related experiences with other TSS. Finally, therapists clearly noted the knowledgeable, friendly, enthusiastic demeanor of the instructors and dedication of the training team. Such qualities facilitated high levels of engagement, comfort, and engagement with the material.

Primary suggestions for improvement included thoughts regarding more discussion of sibling management during therapy sessions. Additional therapists commented that more time was needed so that more frequent breaks could occur. Therapists noted that the speed of presentation was somewhat quick at times and participant chairs were somewhat uncomfortable. Finally, therapists suggested that pre-workshop training with parents may be beneficial as well as a frequently asked question sheet detailing commonly encountered problems during SCIT implementation and solutions. Taken together, SCIT therapists were highly satisfied with the workshop trainings thereby contributing to high levels of acceptability of the intervention among TSS.

TAU workshop feedback. TAU therapists also provided feedback upon TAU workshops following each training. Quantitative analysis indicated that SCIT therapists were significantly more satisfied with SCIT workshops as compared to TAU therapists. Such significant differences were likely to have occurred for multiple reasons. Namely, given that SCIT and TAU therapists were recruited from the same agency, many TAU therapists had learned of the SCIT 
training and had hoped to take part in the SCIT training group. Upon arrival to the workshop, consent procedures were explained and all TAU therapists learned of their assignment to the attention control group. Therefore it is likely that reactivity to the control condition may have negatively skewed perceptions of participation in the TAU workshop.

Despite participation in the attention control workshop, TAU therapists continued to report high levels of satisfaction following the training. Specifically, TAU therapists also appreciated the hands-on activities and interactive nature of the workshop. Therapists enjoyed learning real-life skills such as relaxation, meditation, and muscle relaxation to apply to everyday scenarios to prevent burn out. Therapists also appreciated the opportunity to openly discuss experiences as a TSS, voice concerns, and hear feedback from TSS from a variety of agency offices regarding experiences as a TSS. Similar to SCIT therapists, TAU therapists also commented upon the friendly, open, nonjudgmental, friendly, and knowledgeable nature of the instructors who successfully sustained participant's interest in workshop material. Finally, therapists also appreciated the openness of the agency to participating in the current evaluation in light of potential change.

TAU therapists noted a variety of areas for potential improvement. Namely, TAU therapist expressed a desire to participate in the SCIT condition or receive training in SCIT. Additionally, TAU therapists commented on the long length of the workshop noting the need for a shortened training. Therapists also commented upon the instructor's lack of comprehensive knowledge regarding the TSS position. Some therapists voiced displeasure regarding the assessment role play scenarios. Finally, therapists noted clear confusion within the agency regarding the purpose of the training and therefore unclear communication to therapists regarding training goals and objectives. It is likely that initial confusion existed among TAU 
therapists due to attempts to keep therapists blind to condition prior to the initiation of the workshop. However, such confusion was quickly addressed during consent procedures and a thorough explanation of the training in context of the larger evaluation helped to clarify the purpose behind procedures. Additionally, TAU therapists received a didactic overview of SCIT during their final, half-day workshop. Taken together, although many TAU therapists had hoped to participate in the SCIT condition, quantitative and qualitative evaluation data suggests high levels of workshop satisfaction and acquisition of useful stress management skills.

Therapist competence. Results indicated that therapists in the SCIT group reported a significantly greater increase in therapist competence on the SSOC following SCIT as compared to TAU therapists over the same time period. Furthermore, a comparison of effect sizes between groups suggest a medium effect of SCIT on therapist competence as compared to a small effect of TAU. As noted in previous literature (Beidas \& Kendall, 2010), the active learning approach (e.g., simulated role-play, feedback, coding of therapist skill) utilized in SCIT workshops may have positively influenced therapist skill acquisition and perceptions of competence.

Additionally, delivery of an evidence-based treatment may have had a positive impact upon SCIT therapist's perceptions of their own sense of competence as compared to therapists implementing treatment as usual. Such findings coincide with previous results indicating changes in child behavior problems in the SCIT condition as compared to the TAU condition. Finally, therapists' average SSOC scores steadily increased over time in the SCIT group as compared to remaining relatively stagnant in the TAU group, indicating the compounding effect of SCIT training and treatment implementation over time on therapist sense of competence. Therefore, it may be possible for SCIT to positively impact therapist turnover in future implementation. Such quantitative results combined with qualitative feedback suggest that heightened feelings of 
therapist competence and effectiveness may contribute to lower job-related stress and lower rates of turnover.

Qualitative analysis themes. Following qualitative analysis of TAU focus group discussions, several key themes emerged indicating therapists' thoughts regarding their experience providing in-home services within the agency as well as the larger context of the wraparound model. Broadly, themes fell within two primary categories: 1) BHRS policies in conflict with expected treatment outcomes and 2) consistency of the BHRS model. Each theme will be discussed below. It should be noted that the aforementioned discussion and accompanying table are based on discussions with 32 individual TAU therapists across the agency. Although noteworthy, it remains important to qualify broad generalization of such responses to the larger BHRS and wraparound system with knowledge of the relatively small sample size and individual experiences of such TSS within their respective BHRS offices.

Referral system. TSS described their position in relation to the BHRS hierarchy as falling at the bottom of the chain of command "in so many ways." As such, the TSS only becomes involved in the referral process following the assignment of the client's age, hours assigned, and location in a primary attempt to fit the client within the TSS's schedule. Thus, TSS commented that up until that point, they have not taken part in nor been made aware of the brief psychological evaluation of the client. Additionally, a lack of multi-method assessment exists as client diagnosis and prescription of service hours is often made based primarily on a parent report based interview. Such division between the referral system and TSS ultimately assigned to provide treatment to the case serves to create disconnect between the therapist's skill and preparation for a given diagnostic presentation and a client's needs. Furthermore, such strong boundaries between staff at different levels of the agency contributes to TSS feelings of 
powerlessness and disconnect from their vital position as the primary line of treatment with such clients.

Policy-based limitations. As previously described, the BHRS service line is primarily modeled within the context of nationally-based wraparound services. Therefore some of the following BHRS policies are reflective of tenants of the broader wraparound model. However, TSS in the current sample noted applications of such policies with regard to the conflict they pose to expectations for providing effective treatment to BHRS clients. For example, although the TSS primarily provides individualized services to the child client, they are restricted from being in a room alone with the child. However, many TSS noted that given the parent's frequent disengagement from treatment procedures, it is not unlikely for the TSS to be alone with the child while the parent engages in other household tasks. Additionally, BHRS policies restrict the TSS from teaching and providing knowledge to the child's parent as such responsibilities are allocated to the masters-level BSC. Such restrictions limit transfer of skill to the parent. TSS additionally commented that communication with the BSC may be limited, thereby negatively affecting the team's ability to provide consistent, unified knowledge to the child's parent. Throughout sessions, TSS must deliver treatment based off the treatment plan created by the BSC. While it is clear that some TSS collaborate with the BSC to devise an appropriate, agreed upon treatment plan, others noted their lack of involvement in the creation of the treatment plan and session-by-session data tracking system whereby progress is measured. Additionally, TSS commented that such data tracking systems may be intricate and at times present unclear operationalizations of target behaviors resulting in invalid or ineffective data. Therefore, a significant focus of the TSS position is dedicated to assessment of behaviors versus modification of such behaviors. 
Consumer model. Although referred to as "clients" in the current evaluation, children and parents in the BHRS system are commonly referred to as "consumers," reflective of the broader consumer-based model. A BHRS child and his or her family are thought to be consuming a service designed by the BSC that, in theory, could be implemented by any TSS. As such, families possess ultimate decision-making power regarding the type of service they receive, where it is delivered, and who provides the service. Across the current evaluation, it was common for SCIT therapists in particular, to report that they were unable to deliver an in-home SCIT session due to families determining that they preferred sessions to be conducted at a park, grocery store, or another recreational outing. Additionally, TSS commonly noted that families can choose to discontinue services or switch therapists at any time leading to a lack of predictability for the TSS and child client.

Role expectations. Another prominent theme that emerged from focus group discussions with BHRS therapists was the broad context of consistency within the BHRS model. TSS commented that role expectations by parents of the TSS were unclear and inconsistent. For example, TSS noted that parents refer to the TSS as a babysitter, helper, friend to the parent and/or client, teacher, mentor, and therapist. Such unclear role attributions lead to a lack of respect for the TSS by parents, misattributions regarding the agent of change, and unclear messages for the child. For example, some TSS noted that children will come to them for help over their parents. In the school-based setting, similar misattributions of the role of the TSS are present. Likely overwhelmed with children of varying needs, teachers often become dependent upon TSS to help in the broader classroom, versus with the individual BHRS child.

Parent involvement and skill transfer. Perhaps the most common theme present across discussions with TAU therapists referred to parent involvement in sessions. Many TSS 
commented that parents were overwhelmingly uninvolved in therapy sessions, often utilizing the time to complete household tasks. Other TSS noted that some parents present as overinvolved, intrusive, and controlling within sessions, leading to therapist frustration and unclear, inconsistent messages delivered between the therapist and family. One possible explanation for such role confusion may be a breakdown in clear, operationalized goals and expectations between the agency, parent, and therapist leading each party to operationalize his or her own expectations for service delivery.

Inconsistent contextual factors. Therapists additionally commented upon a number of logistical and contextual factors inherent within the TSS position that contributed to their stress level and negatively impacted the quality of treatment provided to consumers. For example, TSS are only paid for hours in which they are able to bill when providing services. However, TSS commonly cited frequent client cancellations and unpredictable lengths of treatment time prior to termination (e.g., family discontinues services, insurance changes) as common reasons for inconsistent opportunities to bill for service. Additionally, partially as a function of in-home service delivery, therapists also commented upon high levels of environmental distractions (e.g., $\mathrm{TV}$, siblings, noise level) as a primary source of interference with effective treatment procedures. Although some distractions are expected in a home environment, the frequency with which therapists cited such interferences suggests potential disconnect between expectations of the role of the TSS between the client's family and agency.

Lack of psycho-education/specialized training. Across TAU discussions, therapists commented upon the activities and methods utilized in service delivery. Although it was clear that TSS were required to attend a number of psycho-educationally based trainings, therapists possessed mixed amounts of knowledge surrounding the use or principles behind evidence-based 
treatment strategies for specific conditions. Although it is acknowledged that the masters-level BSC is likely to possess greater knowledge and understanding of evidence-based treatment strategies, such lack of knowledge may hinder TSS ability to utilize such foundational understanding to alter treatment strategies in real-time during individual sessions.

Supervision. In BHRS, regular supervision of TSS occurs in two primary ways: 1) during group supervision with clinical supervisors or 2) during joint involvement of the BSC and TSS with a client. TSS commented upon supervision received in the context of BHRS services. It was commonly noted that the frequency with which group supervision occurs primarily depends upon the quantity of BHRS hours provided. In practice, TSS commented upon the inconsistency with which BSC's are jointly involved in sessions with BHRS clients. While TSS noted that some BSC's are heavily involved and clearly dedicated to providing high quality care, other TSS noted difficulties with communication and engagement of the BSC. In the context of group supervision, some TSS commented that helpful strategies with specific consumers are discussed. Other TSS noted that group supervision provides few effective treatment skills and becomes focused on therapist's specific frustrations with given clients. Overall, some therapists noted a lack of supervision time and accountability for effective supervision within the agency.

A final theme that emerged from discussions with TAU therapists appeared to illustrate the result of the aforementioned themes upon therapists and families. First, therapists commented upon an overall lack of progress throughout treatment. At times, TSS noted changes in the child when the TSS was present but no changes in the parent. As a result, TSS commented that such lack of progress teaches TSS not to invest more in treatment delivery than the parent. Next, high levels of job stress as a result of poor pay, a lack of predictability, no incentives, a lack of TSS 
and management evaluation, combined with an overall lack of support, result in high rates of therapist burn out and turnover.

Although the focus of the aforementioned discussion centered upon understanding treatment as usual, TAU therapists also provided a few suggestions for improvement including the ability to talk about cases with individuals informed about clients, evaluations for management, and restricting the length of therapist travel.

SCIT treatment feedback. Following SCIT implementation, SCIT therapists provided reflections upon SCIT implementation. Prior to SCIT implementation, therapists noted a variety of desired client outcomes including increased appropriate play skills, improved social interaction, increased compliance, decreased aggression, increased confidence, improved transfer of skill to the parent, and a more positive parent-child relationship. Following SCIT implementation, therapists noted a variety of changes in the client, his or her parent, in the parent-child relationship, and within themselves. Namely, therapists indicated that clients demonstrated more appropriate play, increased compliance, increased communication and use of language, increased attention to task, less intense temper tantrums, increased eye contact, and increased confidence. Therapists also noted changes in parent skills including increased labeled praise, more effective use of commands, more consistent follow through, and greater awareness of negative talk. Such improvements were reflected within the parent-child relationship as therapists noted more positive and effective interactions between the parents and clients. Therapists also reported that parents became more aware of positive child behaviors and noted increased involvement of the parent within sessions. Some therapists noted that parents did not change. Additionally, therapists commented upon changes within themselves including increased skills in implementing SCIT and in facilitating interactions. Therapists also commented upon 
increased awareness of negative talk, increased attention to positive child behaviors, and tendency to use the PRIDE skills with individuals beyond the client. Finally, therapists noted increased attention to and realization regarding the importance of the parent-child interaction.

Despite such positive reflections, therapists also commented upon key barriers to SCIT implementation. Such barriers included parent and child health problems, interference of siblings during sessions, cancellation of BHRS services, consistency of involvement between BSC, parents, and TSS, reducing parent negative talk, and distractions within the environment. As a result, therapists suggested discussions with parents prior to program initiation, a shorter time in between trainings one and two, toys for older children, greater BSC training, and alternative ways to track homework to increase homework compliance. Taken together, such feedback indicates that SCIT therapists were highly satisfied with SCIT implementation. The program provided therapists with effective skills to be used across child clients with a diverse array of difficulties. Furthermore, benefits of the program extended beyond child clients to parents and therapists providing BHRS services with an effective operationalization of skill transfer between therapists and parents and improving consistency within the treatment team.

\section{Limitations}

A variety of limitations present in the current study are important to consider in light of the aforementioned findings. Such limitations can be divided into three broad themes including 1) agency preparation, 2) methodological limitations, and 3) implementation-based limitations.

Agency preparation. The current study was conducted in conjunction with a large community-mental health agency that serves as a primary provider of wraparound services.

Despite a long-standing partnership between the research team and the agency, a research project similar to the scale of the current evaluation had never previously been conducted within the 
agency-based wraparound system of care. Following implementation of the first SCIT training workshop, the impact of SCIT upon treatment service delivery, therapists, agency hierarchy, and agency culture quickly became apparent to the research team and agency management. As a result, SCIT TSS trainings, assessment, and implementation were paused and no SCIT treatment nor evaluation occurred. During this time, a number of key procedures were implemented to improve communication between the research team and the agency and streamline SCIT implementation. First, SCIT-based treatment goals were created in correspondence with standard agency treatment plans. Second, masters-level supervisors of SCIT TSS were trained in a oneday, intensive SCIT overview and asked to deliver the first two sessions of SCIT in which parents are taught SCIT skills. Such changes were made due to BHRS boundaries between bachelor's-level TSS whose primary responsibility falls in delivering interventions and master's level BSC's in charge of teaching parents new skills. Third, a second, day-long SCIT training was added to the training sequence in order to increase skill acquisition and therapist confidence in delivering SCIT sessions. Fourth, an assessment of child behavior problems from the parents perspective was added to assessment measures. Fifth, an addendum was added to the SCIT manual stating that should a child become aggressive toward parents or therapists during SCIT sessions, parents or therapists must face the wall and turn their back toward the child for approximately 30 seconds or until the child is calm, while the therapist is positioned in front of the parent. During such procedures, parents had to remain in the room at all times, to comply with BHRS requirements. Finally, the physical guide used in ADI when children fail to independently comply with a command was operationalized as a gentle physical movement of the child's hand within a five second window in order to minimize physical contact with the child. Sixth, agency management became more heavily involved in recruitment of therapists for 
both SCIT and TAU workshops by contacting individual agency offices and requesting that participation in the evaluation be offered to therapists with eligible clients.

Methodological limitations. Although necessary for successful implementation, the aforementioned pause resulted in a variety of negative methodological repercussions primarily among therapists in the first SCIT group $(n=18)$. Namely, such therapists and their respective clients experienced mixed messages regarding the initiation of SCIT. While most of these therapists had not yet begun SCIT immediately following the first workshop, many had begun discussing procedures with their clients. Following discussion with agency management, such therapists were told that a second, full-day SCIT workshop would be implemented seven weeks following the first workshop at which point formal SCIT implementation would begin. Finally, therapists were asked to attend a third, half-day evaluation workshop seven weeks following the second workshop to provide evaluations of the SCIT program. Additionally, as previously discussed, a proportion of SCIT therapists never had appropriate clients with whom to conduct SCIT (e.g., client dropped out of services in between therapist recruitment and the first workshop) and therefore, were unable to implement the intervention. The majority of therapists never assigned an appropriate client were from this first SCIT group. Due to the interconnected nature of such attrition between clients and therapists, therapist and client within group attrition are likely to be confounded by the opposing group. Additionally, given the addition of the parent assessment of child behavior problems following the initial SCIT workshop, consistent procedures had not been determined and the majority of these therapists were unable to collect a measure of child behavior problems from the parents' perspective following this time point. Such missing measures contributed to smaller sample sizes and decreased power to detect changes in children's behavior problems from the parent's perspective. 
A variety of recruitment procedures were utilized in the current evaluation. Prior to the first workshop, the primary researcher contacted therapists who indicated interest in study participation. A coin flip was used to determine assignment of eligible therapists to predetermined SCIT and TAU workshop dates. Recruitment by corporate-level agency personnel also occurred. However, such lack of consistent participant randomization to condition may have contributed to groups with unequal levels of behavior problems prior to workshop one. Children in the SCIT group entered the study with significantly higher levels of behavior problems as compared to children in the TAU group. As a result, regression to the mean may have contributed to improvement in child behavior between groups.

Additionally, order effects inherent in conducting two separate workshops prior to full SCIT implementation may have impacted results. Although quadratic analyses have been utilized in other PCIT studies to examine the impact of study condition on treatment effects (Mersky, Topitzes, Grant-Savela, Brondino, \& McNeil, 2014), the availability of only three measurement points and small sample size precluded the use of quadratic analyses in the current study (A. Metzger personal communication, April 16, 2016, N. Turiano personal communication, April 26, 2016). Furthermore, the use of such approaches with the use of small sample sizes may have resulted in an overfit model that may not be likely to replicate in a separate sample (Babyak, 2004). Ultimately, the focus of the current study aimed to evaluate the full SCIT training, of which material taught in the second workshop intricately builds upon material from the first workshop. In the current study, workshops were only conducted seven weeks apart for logistic reasons.

Given that TAU and SCIT therapists worked in the same agency locations and may have shared a single BSC trained in SCIT, treatment spillover between groups is likely to have 
occurred. Therefore, limitations regarding attributions of causality to the condition alone must be mentioned. Ultimately, 19 participants who attended at least one workshop had been randomly assigned to study condition. Following the first workshop, agency management assisted in participant recruitment by assigning interested, eligible therapists to condition. Therefore, it is likely that the overall lack of randomization of assignment resulted in significant differences between groups on unmeasured variables.

As previously described, both therapist and client attrition resulted in decreased sample size, decreased power, and increased chance of type II error, limiting the chance to detect effects that may be present. Perhaps the most noteworthy difference between groups was present between ECBI scores examining child behavior problems at the day one workshop between SCIT and TAU groups with children in the SCIT condition demonstrating significantly higher scores as compared to children in the TAU condition. Therefore, it is possible that regression to the mean may have occurred among SCIT clients naturally resulting in lower ECBI scores over time. Such differences may have occurred as a function of a lack of true randomization to condition across participants.

An analysis of demographic variables at the day one workshop indicated significant differences between groups in the area of more previous training among TAU therapists. While such results may theoretically indicate that TAU therapists are better prepared to serve a wider variety of clients with more diverse needs, such explanation is not likely. Many therapists between groups indicated an inability to remember all trainings previously attended and some did not indicate hours in conjunction with training titles. Therefore, due to the amount of missing data, it is difficult to discern whether actual differences in therapist preparation existed between 
groups. Beyond this single variable, no other demographic differences between groups were detected in either therapists or clients.

Finally, given that the current study was primarily based in quantitative analysis, qualitative analyses were conducted informally. Although the research team attempted to remain as unbiased as possible during focus group interviews and feedback assessments, it is impossible to rule out the influence of potential bias from the collection and analysis of qualitative data in the current investigation.

Implementation-based limitations. In addition to a variety of agency-based and methodological limitations present in the current study, implementation-based limitations also negatively impacted SCIT implementation. First, SCIT families in the current study had been receiving treatment as usual with their TSS for varying lengths of time prior to SCIT implementation. While treatment as usual is primarily focused upon treatment with the individual child within which the parent is peripherally and often inconsistently involved, the sudden initiation of SCIT into the previously dyadic model necessitated high levels of parental time and involvement. Although some therapists reported the relative ease with which parents adapted to such changes, others were met with significant resistance (e.g., lack of parent involvement in sessions, difficulties with parent homework completion), thereby limiting the impact of SCIT upon the child and family. Additionally, BHRS policy regulations specify that a child may either have outpatient or wraparound, home-based therapy services. Children in wraparound services may be significantly different from children involved in outpatient services and therefore the current results may not generalize to this population. Finally, it is possible for children in BHRS services to have multiple TSS (e.g., home-based TSS, school-based TSS) 
depending upon variables such as diagnosis and severity of impairment. Such information was unknown in the current study but may have influenced outcomes between groups.

\section{Future Directions}

Despite a variety of limitations, results of the current study are noteworthy for a number of reasons. First, the current study represents the first full scale implementation of SCIT in a community-based setting. Secondly, this study presents a preliminary analysis of qualitative focus group interviews with treatment as usual therapists. Promising results regarding the impact of SCIT on decreasing the intensity of child disruptive behavior were present. Additionally, qualitative analysis of SCIT therapist feedback to the intervention indicated high levels of treatment acceptability. However, power was limited in the current investigation due to high attrition rates and small sample sizes. Therefore, future research should evaluate a larger scale implementation of SCIT to account for high therapist and client attrition rates. Additionally, SCIT should be implemented with new, eligible families entering the BHRS system. Such an approach may decrease client resistance experienced in the current study due to switching from services as usual to the intensive, parent-focused SCIT model. Randomization of therapist to condition should also be used to decrease the chances of pretreatment differences in child behavior problems. Alternatively, a blocking or matching approach may be used when assigning therapists to workshops.

It is critical to consider treatment fidelity in SCIT implementation. Managed Care Organizations may consider investing in mobile technology devices whereby a SCIT-trained supervisor at a nearby office randomly watches a SCIT session to ensure treatment fidelity. Alternatively, therapists could be required to comment upon each component of SCIT sessions in session notes. Finally, therapist incentives may also be considered in an effort to decrease 
therapist premature drop-out and turnover. Entry into lotteries for gift-cards or prizes may be used when therapists reach certain aspects of treatment and larger incentives may be provided when therapists complete a full course of SCIT with a family.

\section{Future implementation of the SCIT model}

Based on the current research, the following SCIT clinical model is proposed. Due to the inability of all therapists to reach mastery in SCIT skills, a four, full-day SCIT training is recommended during which all therapists and supervisors must master both CDI and ADI skills. Training days should ideally occur within immediate succession or within two weeks of one another. SCIT therapists must be accompanied by their immediate supervisors who participate in the training alongside the SCIT therapist or obtain the same training in a supervision group. Multiple trainers and assistants are needed to provide individual feedback and coaching. Additionally, SCIT supervisors must participate in an additional, supervisor-focused training in which supervisors are taught how to introduce SCIT and troubleshoot concerns with skeptical parents. Supervisor trainings should focus on discussion and practice of the two initial teach sessions. Following completion of all four training days, SCIT supervisors may become "training certified". SCIT programs should consider the possibility of expanding the model to allow TSS to also provide didactic training and coaching to parents.

Based on data in the current study indicating that parents were able to meet CDI mastery in approximately eight sessions, SCIT should include a minimum of eight sessions over no shorter than a four week period. SCIT success can be defined as a decrease in ECBI scores to within normal limits (below 114) combined with parent mastery of both CDI and ADI phases. Therefore, the ECBI should be administered at least three times (e.g., prior to, during, and following SCIT) to the child's parent. Parents should be expected to achieve CDI mastery before 
moving on to the ADI phase of treatment. Upon achievement of such criteria, the treatment team may reassess the value of continuing with SCIT. A graduation session with the family upon successful fulfillment of SCIT criteria should be held.

Due to significant concerns managing sibling behavior during SCIT implementation with the target child in the current study, one SCIT-trained therapist and a second staff member should be present during all SCIT sessions. One therapist is designated as the lead therapist who implements SCIT with the parent and target child, while the other staff member provides childcare to siblings and assists in managing additional distractions. Ideally, SCIT therapists should assist a lead therapist on a SCIT case prior to becoming a lead therapist themselves. Furthermore, SCIT therapists may become certified in SCIT following all four training days and complete implementation with one case. Supervisors may become clinically certified after completion of all four training days and implementation of one case. SCIT supervisors may become SCIT trainers by receiving additional training with master SCIT trainers. Regular consultation between SCIT therapists and SCIT supervisors must occur. Furthermore, a minimum of three consultation calls must occur between SCIT trainers and SCIT supervisors in order for a SCIT case to be considered complete.

Despite the promising results in the current study, it should be acknowledged that SCIT remains a low-level form of intervention. It is anticipated that SCIT would contribute to a larger PCIT-based wraparound service, as proposed by Dr. Cheryl McNeil during the Parent-Child Interaction Therapy Conference (October, 2015). Within this model, children between the ages of two to nine years with disruptive behavior would participate in varying levels of PCIT-based intervention potentially including SCIT, once-weekly PCIT in an outpatient setting, and TeacherChild Interaction Therapy (TCIT; Lyon, Gershenson, Farahmand, Thaxter, Behling, \& Budd, 
2009; Tiano \& McNeil, 2006). Although no research has been conducted to examine the impact of such a comprehensive, intensive model, the vast literature on PCIT suggests that a PCIT wraparound service could result in highly effective treatment for children with severe disruptive behavior as it provides a cohesive model for primary caregivers and providers.

\section{Summary}

The current study represents the first empirical evaluation of Staff-Child Interaction Therapy as implemented by bachelor's level, in-home therapists involved in the wraparound system of care. Results of the current study indicate promising effects of SCIT as a form of treatment delivery in in-home wraparound service. Converging results across each of the dependent variables suggest that SCIT may improve children's behavior and therapist sense of competence while serving as a highly acceptable, cost-effective means of treatment for children and parents involved in wraparound services. However, findings are considered preliminary due to significant methodological limitations including low sample size, high levels of therapist and client attrition, and significantly different pretreatment means on the ECBI. Additional research using larger samples and stronger methodology must be conducted in order for more definitive conclusions to be reached. 


\section{References}

Abidin, R. R. (1990). Parenting Stress Index / Short Form. Lutz, FL: Psychological Assessment Resources, Inc.

Achenbach, T. M., \& Rescorla, L. A. (2000). Manual for ASEBA preschool forms \& profiles. Burlington, VT: University of Vermont, Research Center for Children, Youth, \& Families. Ainsworth, M., Blehar, M., Waters, E., \& Wall, S. (1978). Patterns of attachment: A psychological study of the strange situation. Hillsdale: Erlbaum.

American Psychological Association. (2006). Evidence-based practice in psychology: APA presidential task force on evidence-based practice. American Psychologist, 61, 271-285.

Amico, K. R. (2009). Percent total attrition: A poor metric for study rigor in hosted intervention designs. American Journal of Public Health, 99, 1567-1575.

An introduction to community data roundtable's behavior health rehabilitation services outcomes dashboard (2015). Retrieved from http://www.communitydataroundtable.org/uploads/8/4/9/3/8493187/cdr_report_on_bhrs_outcom es.pdf.

Anderson, J. A. (2011). Patterns of school functioning over time among students served in a system of care. Remedial and Special Education, 32, 482-495.

Aragona, J., \& Eyberg, S. (1981). Neglected children: Mother's report of child behavior problems and observed verbal behavior. Child Development, 52, 596-602.

Arain M., Campbell M.J., Cooper C.L., Lancaster G.A. (2010). What is a pilot or feasibility study? A review of current practice and editorial policy. BMC Medical Research Methodology, 10, 67.

Babyak, M. A. (2004). What you see may not be what you get: A brief, nontechnical introduction to overfitting in regression-type models. Psychosomatic Medicine, 66, 411-421. 
Baumann, A. A., Powell, B. J., Kohl, P. L., Tabak, R. G., Penalba, V., Proctor, E. K., .. \& Cabassa, L. J. (2015). Cultural adaptation and implementation of evidence- based parent-training: A systematic review and critique of guiding evidence. Children and Youth Services Review, 53, $113-120$.

Baumrind., D. (1971). Current patterns of parental authority. Developmental Psychology Monograph, 4, 1-103.

Bazron, B.J. (2012). Statewide implementation of wraparound. Retrieved http://www.tapartnership.org/docs/Statewide_Implementation_of_Wraparound_February_2012.p df.

Beidas, R. S., \& Kendall, P. C. (2010). Training Therapists in Evidence-Based Practice: A critical review of studies from a systems-contextual perspective. Clinical Psychology: Science and Practice, 17(1), 1-30. doi:10.1111/j.1468-2850.2009.01187.x

Bertram, R.M., Suter, J., \& Bruns, E., \& O’Rourke, K. (2011). Implementation research and wraparound literature: Building a research agenda. Journal of Child and Family Studies, 20 (6), 713-726.

Beveridge, R. M., Fowles, T. R., Masse, J. J., McGoron, L., Smith, M. A., Parrish, B. P., ... Widdoes, N. (2015). State-wide dissemination and implementation of parent-child interaction therapy (PCIT): Application of theory. Children \& Youth Services Review, 48, 38-48.

Bickman, L., Smith, C. M., Lambert, E. W., \& Andrade, A. R. (2003). Evaluation of a congressionally mandated wraparound demonstration. Featured article. Journal of Child and Family Studies, 12, $135-156$.

Billingham, S.A., Whitehead, A.L. \& Julious, S.A. (2013) An audit of sample sizes for pilot and feasibility trials being undertaken in the United Kingdom registered in the United Kingdom 
Clinical Research Network database. BMC Medical Research Methodology, 13, 104.

Boggs, S.R., Eyberg, S.M., \& Reynolds, L. (1990). Concurrent validity of the Eyberg Child Behavior Inventory. Journal of Clinical Child Psychology, 19, 75-78. doi:10.1207/s15374424jccp1901_9

Borrego, Jr., J., Anhalt, K., Terao, S. Y., Vargas, E. C., Urquiza, A. J. (2006). Parent-child interaction therapy with a spanish-speaking family. Cognitive and Behavioral Practice, 13, 121-133.

Bowen, D.J., Kreuter, M., Spring, B., Cofta-Woerpel, L., Linnan, L., Weiner, D., Bakken, S., Patrick Kaplan, C., Squiers, L., Fabrizio, C., Fernandes, M. (2009). How we design feasibility Studies. American Journal of Preventive Medicine, 36(5), 452-457.

Brestan, E., Eyberg, S.M., Boggs, S. \& Algina, J. (1997). Parent-child interaction therapy: Parent perceptions of untreated siblings. Child \& Family Behavior Therapy, 19, 13-28.

Bronfenbrenner, U. (1979). The Ecology of Human Development: Experiments by Nature and Design. Harvard University Press, Cambridge, Massachusetts.

Brown, S., Valente, J.R. Wild, R.C., Whitaker, D.J., Galanter, R., Dorsey, S., \& Stanley, J. (2012). Utilizing bench marking to study the effectiveness of parent-child interaction therapy implemented in a community setting. Journal of Child and Family Studies, 21, 1041-1049.

Bruns, E. J., Walker, J. S., Zabel, M., Matarese, M., Estep, K., Harburger, D., \& Mosby, M. (2010). Intervening in the lives of youth with complex behavioral health challenges and their families: The role of the wraparound process. American Journal of Community Psychology, 46, 314-331.

Bruns, E. J., Rast, J., Walker, J. S., Peterson, C. R., \& Bosworth, J. (2006). Spreadsheets, service providers, and the statehouse: Using data and the wraparound process to reform systems for children and families. American Journal of Community Psychology, 38, 201-212. 
Bruns, E. J., Burchard, J. D., Suter, J. C., Leverentz-Brady, K., \& Force, M. M. (2004). Assessing fidelity to a community-based treatment for youth: The wraparound fidelity index. Journal of Emotional and Behavioral Disorders, 12, 79-89.

Bruns, E. J., Walker, J. S., \& The National Wraparound Initiative Advisory Group. (2008). Ten principles of the wraparound process. In E. J. Bruns \& J. S. Walker (Eds.), The re-source guide to wraparound. Portland, OR: National Wraparound Initiative, Research and Training Center for Family Support and Children's Mental Health, Portland State University.

Bruns, E. J., Walker, J. S., Zabel, M., Matarese, M., Estep, K., Harburger, D., ... \& Pires, S. A. (2010). Intervening in the lives of youth with complex behavioral health challenges and their families: The role of the wraparound process. American Journal of Community Psychology, 46(3), 314331.

Burchard, J., Bruns, E.J., \& Burchard, S.N. (2002). The Wraparound Approach. In Burns, B. and Hoagwood, K. (Eds.), Community Treatment for Youth: Evidence- Based Treatment for Severe Emotional and Behavioral Disorders. Oxford: Oxford University Press.

Burchard, J. D., Burchard, S. N., Sewell, R., \& VanDenBerg, J. (1993). One Kid at a Time: Evaluative case studies and descriptions of the Alaska Youth Initiative Demonstration Project. Washington, DC: SAMHSA Center for Mental Health Services.

Child \& adolescent needs \& strengths (CANS-MH Manual). (2008). Retrieved https://www.magellanprovider.com/media/11838/cans-mhmanual.pdf.

Capage, L. C., Bennett, G., \& McNeil, C. B. (2001). A comparison between African American and Caucasian children referred for treatment of behavior problems. Child and Family Behavior Therapy, 23, 1-14.

Carpenter, A.L., Puliafico, A.C., Kurtz, S.M.S., Pincus, D.B., \& Comer, J.S. (2014). Extending Parent- 
Child Interaction Therapy for early childhood internalizing problems: New advances for an overlooked population. Clinical Child and Family Psychology Review, 17, 340-356.

Chaffin, M., Funderbunk, B., Bard, D., Valle, L.A., \& Gurwitch, R. (2011). A combined motivation and parent-child interaction therapy package reduces child welfare recidivism in a randomized dismantling field trial. Journal of Consulting and Clinical Psychology, 79, 84-95. doi:10.1037/a0021227

Chaffin, M., Silovsky J. F., Funderburk, B., Valle, L. A., Brestan, E. V., Balachova, T., Bonner, B. L, et al. (2004). Parent-child interaction therapy with physically abusive parents: Efficacy for reducing future abuse reports. Journal of Consulting and Clinical Psychology, 72(3), 500-510.

Chaffin, M., Valle, L.A., Funderburk, B., Gurwitch, R., Silovsky, J., Bard, D., et al. (2009). A motivational intervention can improve retention in PCIT for low-motivation child welfare clients. Child Maltreatment, 14(4) 356-368.

Chamberlain, P., \& Reid, J. B. (1998). Comparison of two community alternatives to incarceration for chronic juvenile offenders. Journal of Consulting and Clinical Psychology, 66(4), 624-633.

Chambless, D. L., Baker, M. J., Baucom, D. H., Beutler, L. E., Calhoun, K. S., Crits-Christoph, P.,... Woody, S. R. (1998). Update on empirically validated therapies, II. The Clinical Psychologist, 51(1), 3-16.

Chase, R. M., \& Eyberg, S. M. (2008). Clinical presentation and treatment outcome for Children with Comorbid Externalizing and Internalizing Symptoms. Journal of Anxiety Disorders, 22. 273-282. doi:10.1016/j.janxdis.2007.03.006

Clark, H. B., \& Clarke, R. T. (1996). Research on the wrap-around process and individualized services for children with multi-system needs. Journal of Child and Family Studies, 5, 1-5. 
Clarke, R. T., Schaefer, M., Burchard, J. D., \& Welkowitz, J. W. (1992). Wrapping community mental health services around children with a severe behavioral disorder: An evaluation of Project wraparound. Journal of Child and Family Studies, 1(3), 241-261.

The Cochrane Effective Practice and Organisation of Care Review Group: Data Collection Checklist (2002). Retrieved from http://epoc.cochrane.org/sites/epoc.cochrane.org/files/uploads/datacollectionchecklist.pdf.

Cocozza, J., \& Skowyra, K. (2000).Youth with Mental Health Disorders: Issues and Emerging Responses. Office of Juvenile Justice and Delinquency Prevention Journal, 7(1), 3-13

Colvin, A., Eyberg, S.M., \& Adams, C. (1999). Restandardization of the Eyberg Child Behavior Inventory. Gainesville: University of Florida, Child Study Laboratory. Retrieved from http://pcit.phhp.ufl.edu/Measures.htm.

Community behavioral health (2014). Retrieved from http://dbhids.org/wpcontent/uploads/2015/10/Manual-for-Review-of-Provider-Personnel-Files-v.1.1-August2014.pdf.

Copp, H. L., Bordnick, P. S., Traylor, A. C., \& Thyer, B. A. (2007). Evaluating wraparound services for seriously emotionally disturbed youth: Pilot study outcomes in Georgia. Adolescence, 42, 2007.

Eisenstadt, T. H., Eyberg, S., McNeil, C. M., Newcomb, K., \& Funderburk, B. (1993). Parent-child interaction therapy with behavior problem children: Relative effectiveness of two stages and overall treatment outcome. Journal of Clinical Child Psychology, 22, $42-51$.

Eyberg, S. M. , Funderburk, B. (2011) Parent-Child Interaction Therapy protocol. PCIT International; Gainesville, FL.

Eyberg, S. M., Funderburk, B. W., Hembree- Kigin, T. L., McNeil, C. M., Querido, J. G., \& Hood, K. 
K. (2001). Parent-child interaction therapy for behavior problem children: One and two year maintenance of treatment effects in the family. Child and Family Behavior Therapy, 23, 1-20.

Eyberg, S.M., Nelson, M. M., \& Boggs, S.R. (2008). Evidence-based treatments for child and adolescent disruptive behavior disorders. Journal of Clinical Child and Adolescent Psychology, $37,213-23$

Eyberg, S.M., Nelson, M.,M., Duke, M., \& Boggs, S.R. (2005). Manual for the Dyadic Parent-Child Interaction Coding System (3rd edition).

Eyberg, S.M., \& Pincus, D. (1999). Eyberg Child Behavior Inventory and Sutter-Eyberg Student Behavior Inventory-Revised: Professional Manual. Odessa, FL: Psychological Assessment Resources.

Fairburn, C. G., \& Cooper, Z. (2011). Therapist competence, therapy quality, and therapist training. Behaviour Research and Therapy, 49(6-7), 373-378. http://doi.org/10.1016/j.brat.2011.03.005

Fernandez, MA, Butler, AM, \& Eyberg, SM. (2011). Treatment Outcome for Low Socioeconomic Status African American Families in Parent-Child Interaction Therapy: A Pilot Study. Child and Family Behavior Therapy, 33(1), 32-48. doi:10.1080/07317107.2011.545011

Fernandez, M., \& Eyberg, S. (2009). Predicting treatment and follow-up attrition in Parent-Child Interaction Therapy. Journal of Abnormal Child Psychology, 37, 431-441. doi:10.1007/s10802008-9281-

Forgatch, M. S., \& Patterson, G. R. (2003). Parent management training - Oregon model: An intervention for antisocial behavior in children and adolescents. In A. E. Kazdin, \& J. R. Weisz (Eds.), Evidence-based psychotherapies for children and adolescents (pp. 241262). New York: Guilford Press.

Fowles, Masse, McGoron, Beveridge, Williamson, Smith, Parish. (in preparation). Home vs. clinic 
based Parent-Child Interaction Therapy (PCIT): Comparative effectiveness and attrition in the context of dissemination and implementation.

Frazier, S. L., Formoso, D., \& Birman, D., \& Atkins, M. S. (2008). Closing the research to practice gap: Redefining feasibility. In the Special Series: Ethical Challenges in Community-Based Research (Eds: C. Johnston \& S. Woody). Clinical Psychology: Science and Practice, 15, 125129.

Funderburk, B.W., Eyberg, S.M., Rich, B., \& Behar, L. (2003). Further psychometric evaluation of the Eyberg and Behar rating scales for parents and teachers of preschoolers. Early Education \& Development, 14, 67-81.

Galanter, R., Self-Brown, S., Valente, J. R., Dorsey, S., Whitaker, D. J., Bertuglia-Haley, M., \& Prieto, M. (2012). Effectiveness of parent-child interaction therapy delivered to at -risk families in the home setting. Child \& Family Behavior Therapy, 34, 177-196.

Gilmore, L., \& Cuskelly, M. (2008). Factor structure of the parenting sense of competence scale using a normative sample. Child: Care, Health and Development, 35(1), 48-55. doi:10.1111/j.13652214.2008.00867.x

Goldfine, M. E., Wagner, S. M., Branstetter, S. A., \& McNeil, C. B. (2008). Parent child interaction therapy: An examination of cost-effectiveness. Journal of Early and Intensive. Behavior Intervention, 5, 119-141.

Gurwitch, R.H., Messer, E., Masse, J., Olafson, E., Boat, B.W., \& Putnam, F.W. (2015). Child-Adult Relationship Enhancement (CARE): An evidence-informed program for children with a history of trauma and other behavioral challenges. Child Abuse \& Neglect, 53, 138-145. doi: 10.1016/j.chiabu.2015.10.016.

Hanf, C. A., (1969). A two-stage program for modifying maternal controlling during mother-child (M- 
C) interaction. Paper presented at the meeting of the Western Psychological Association, Vancouver.

Henggeler, S.W. \& Lee, T. (2003). Multisystemic treatment of serious clinical problems. In A.E.Kazdin \& J.R. Weisz (Eds.). Evidence Based Psychotherapies for Children \& Adolescents (pp. 301322). New York: The Guilford Press.

Henggeler, S. W., Melton, G. B. and Smith, L. A. (1992). Family preservation using multisystemic therapy: an effective alternative to incarcerating serious juvenile offenders. Journal of Consulting and Clinical Psychology, 60, 953-961.

Herschell, A.D., McNeil, C.B., Urquiza, A.J., McGrath, J.M., Zebell, N.M., Timmer, S.G., et al. (2009). Evaluation of a treatment manual and workshops for disseminating, parent-child interaction therapy. Administration and Policy in Mental Health and Mental Health Services Research, 36(1) 63-81.

Hogan, M. F., et al.,(2003, July 22). The President's New Freedom Commission on Mental Health. Retrieved from https://store.samhsa.gov/shin/content/SMA03-3831/SMA03-3831.pdf.

Hood, K., \& Eyberg, S.M. (2003). Outcomes of parent-child interaction therapy: Mothers' reports on maintenance three to six years after treatment. Journal of Clinical Child and Adolescent Psychology, 32, 419-429. doi:10.1207/S15374424JCCP3203_10

Huey, S.J., Henggeler, S.W., \& Brondino, M.J., \& Pickrel, S.G. (2000). Mechanisms of change in Multisystemic therapy: Reducing delinquent behavior through therapist adherence and improved family and peer functioning. Journal of Consulting and Clinical Psychology, 68, 451-467.

Hyde, K.L., Burchard, J.D., \& Woodworth, K. (1996). Wrapping services in an urban setting. Journal of Child and Family Studies, 5, 67-82.

Johnston, C., \& Mash, E.J. (1989). A measure of parenting satisfaction and efficacy. Journal of 
Clinical Child Psychology, 18, 167-175.

Justice Policy Institute (2014). Sticker shock: Calculating the full price tag for youth incarceration.

Retrieved

http://www.justicepolicy.org/uploads/justicepolicy/documents/sticker_shock_final_v2.pdf

Kaminski, J. W., Valle, L. A., Filene, J. H., \& Boyle, C. (2008). A meta-analytic review of components associated with parent training program effectiveness. Journal of Abnormal Child Psychology, $36,567-589$.

Kamradt, B. (2011). Individualizing care for children with complex needs through developing a comprehensive service array and provider network [PowerPoint slides]. Retrieved from http://www.nwi.pdx.edu/webinars/Webinar11.pdf.

Kazdin, A. (2003). Research design in clinical psychology. Boston, MA: Allyn \& Bacon.

Kazdin, A. E., Bass, D., Siegel, T. \& Thomas, C. (1989). Cognitive-behavioral treatment and relationship therapy in the treatment of children referred for antisocial behavior. Journal of Consulting and Clinical Psychology, 57, 522-535.

Kendall, P. C. (1998). Directing misperceptions: Researching the issues facing manual-based treatments. Clinical Psychology: Science and Practice, 58, 729-740.

Kingston, J. (2004). Conducting feasibility studies for knowledge based systems. Knowledge-based systems. Knowledge Based Systems, 17(2-4), 157-164.

Kraemer, H.C., Mintz, J., Noda, A., Tinklenberg, J., Yesavage, J.A. (2006). Caution regarding the use of pilot studies to guide power calculations for study proposals. Archives of General Psychiatry, $63,484-489$.

Krivelyova, A., Sukumar, B., Stephens, R., \& Freeman, K. (2007, March). Treatment costs for children and families receiving parent-child interaction therapy or services as usual in a system of care 
setting. Poster presented at the 20th Annual Research Conference, Tampa, FL.

Lancaster, G.A., Dodd, S., Williamson, P.R. (2004). Design and analysis of pilot studies: recommendations for good practice. Journal of Evaluation in Clinical Practice, 10, 307-312.

Lenhard, W. \& Lenhard, A. (2016). Calculation of Effect Sizes. available: http://www.psychometrica.de/effect_size.html. Bibergau (Germany): Psychometrica.

Lenze, S., Pautsch , J., \& Luby, J. (2011). Parent-child interaction therapy emotion development: A novel treatment for depression in preschool children. Depression and Anxiety, 28, 153-159.

Leung, C., Tsang, S., Heung, K., \& Yiu, I. (2009). Effectiveness of Parent-Child Interaction Therapy (PCIT) among Chinese families. Research on Social Work Practice, 19, 304-313.

Lyon, A.R., \& Budd, K.S. (2010).A community mental health implementation of Parent-Child Interaction Therapy (PCIT). Journal of Child and Family Studies, 19, 654-668.

Lyon, A. R., Gershenson, R. A., Farahmand, F., Thaxter, P., Behling, S., \& Budd, K. S. (2009). Effectiveness of Teacher-Child Interaction Training (TCIT) in a preschool setting. Behavior Modification, 33, 855-884.

Masse, J., McNeil, C. B., Wagner, S. M., \& Quetsch, L. B. (accepted). Examining the Efficacy of Parent-Child Interaction Therapy with Children on the Autism Spectrum. Journal of Child and Family Studies.

Matos, M., Bauermeister, J., \& Bernal, G. (2009). Parent-child interaction therapy for Puerto Rican preschool children with ADHD and behavior problems: A pilot efficacy study. Family Process, 48, $232-252$.

McCabe, K. \& Yeh, M. (2009). Parent-child interaction therapy for Mexican Americans: A randomized clinical trial. Journal of Clinical Child \& Adolescent Psychology, 38, 753 - 759.

McMahon, R. J., \& Forehand, R. L. (2003). Helping the noncompliant child: Family-based treatment 
for oppositional behavior $\left(2^{\text {nd }}\right.$ Ed.), New York: The Guilford.

McNeil, C., \& Hembree-Kigin, T. L. (2010). Parent-child interaction therapy (2nd ed.). New York, NY US: Springer Science + Business Media.

McNeil, C. B., Eyberg, S., Eisenstadt, T. H., Newcomb, K., \& Funderburk, B. (1991). Parent-child interaction therapy with behavior problem children: Generalization of treatment effects to the school setting. Journal of Clinical Child Psychology, 20, 140-151.

McNeil, C. B.,Filcheck, H. A.,Greco, L. A.,Ware, L. M.\& Bernard, R. S.(2001).Parent-child interaction therapy: Can a manualized treatment be functional? The Behavior Analyst Today, 2, 106-115.

Mersky, J. P., Topitzes, J., Grant-Savela, S. D., Brondino, M. J., McNeil, C. B. (2014). Adapting parent-child interaction therapy to foster care: Outcomes from a randomized trial. Research on Social Work Practices, 1-11. doi: 10.1177/1049731514545023.

National Institute for Health Research (2016). Retrieved from http://www.nets.nihr.ac.uk/glossary?result_1655_result_page=F.

Newcomer, K.E., Hatry, H.P., \& Wholey, J. S. (2015). Handbook of practical program evaluation, $4^{\text {th }}$ Ed. Hoboken, NJ: John Wiley \& Sons.

Norman, M., \& McNeil, C. B. (2015). Staff Child Interaction Therapy. Unpublished manuscript, Department of Psychology, West Virginia University, Morgantown, West Virginia.

Ollendick, T. H., \& King, N. J. (2004). Empirically supported treatments for children and adolescents: Advances toward evidence-based practice. In P. M. Barrett \& T. H. Ollendick (Eds.), Handbook of interventions that work with children and adolescents: Prevention and treatment (pp. 1-26). Chichester: John Wiley \& Sons, Ltd. and treatment (pp. 1-26). Chichester: John Wiley \& Sons, Ltd.

Oser, C., Knudsen, H., Staton-Tindall, M., Leukefeld, C. (2009). The adoption of wraparound services 
among substance abuse treatment organizations serving criminal offenders: The role of a women-specific program. Drug and Alcohol Dependance, 103, S82-S90.

Painter, K. (2012). Outcomes for youth with severe emotional disturbance: A repeated measures longitudinal study of a wraparound approach of service delivery in systems of care. Child \& Youth Care Forum, 41, 407-425. doi: 10.1007/s10566-011-9167-1

Parent-Child Interaction Therapy International. (2016). Retrieved from pcit.org.

Pearl, E., Thieken, L., Olafson, E., Boat, B., Connelly, L., Barnes, J., et al. (2012). Effectiveness of community dissemination of parent-child interaction therapy. Psychological trauma: Theory, Research, Practice, and Policy, 4, 204-213.

Pennsylvania Department of Human Services (2015). Medical Assistance Coverage of Applied Behavioral Analysis (ABA) for Children and Adolescents with Autism Spectrum Disorder (ASD). Presentation presented for the BH-MCO and County HealthChoices Contacts as a WEBEX Presentation.

Performance standards (2012). Retrieved from http://www.ccbh.com/pdfs/Providers/performanceStandards/BHRSforAutismSpectrumDisorder s.pdf.

Phillips, J., Morgan, S., Cawthorne, K., \& Barnett, B. (2008). Pilot evaluation of parent-child interaction therapy delivered in an Australian community early childhood clinic setting. Australian and New Zealand Journal of Psychiatry, 42, 712-719.

Pincus, D.B., Santucci,, L.C., Ehrenreich, J., \& Eyberg, S.M. (2008). The implementation of modified parent-child interaction therapy for youth with separation anxiety disorder. Cognitive and Behavioral Practice, 15, 118-125. 
Recovery toolkit. (2011). Retrieved from http://www.vbhpa.com/provider/info/clinical_ut/BHRS_Recovery_Toolkit.pdf.

Reitman D, McMahon, R.J. (2012). Constance "Connie" Hanf (1917-2002): The mentor and the model. Cognitive and Behavioral Practice, 20, 106-116.

Sanders, M. R., Markie-Dadds, C., Tully, L. A., \& Bor, W. (2000). The Triple P - Positive Parenting Program: A comparison of enhanced, standard and self-directed behavioural family intervention for parents of children with early onset conduct problems. Journal of Consulting and Clinical Psychology, 68, 624-640.

Schuhmann, E. M., Foote, R. C., Eyberg, S. M., Boggs, S. R., \& Algina, J. (1998). Efficacy of parentchild interaction therapy: Interim report of a randomized trial with short-term maintenance. Journal of Clinical Child Psychology, 27, 34-45.

Shaw, B. F., Elkin, I., Yamaguchi, J., Olmsted, M., Vallis, T. M., Dobson, K. S., . . Imber, S. D. (1999). Therapist competence ratings in relation to clinical outcome in cognitive therapy of depression. Journal of Consulting and Clinical Psychology, 67(6), 837-846. doi:10.1037/0022006x.67.6.837

Silverman, W.K., Ortiz, C., Visersvaran, C., Burns, B.J., Kolko, D.J., Putnam, F.W., \& Amaya-Jackson L. (2008). Evidence-Based Psychosocial Treatments for Children and Adolescents Exposed to Traumatic Events. Journal of Clinical Child \& Adolescent Psychology, 37, 156-183. doi: $10.1080 / 15374410701818293$

Sniehotta, F. F., Dombrowski, S.U., Avenell, A., Johnston, M., McDonald, S., \& Murchie, P., et al. (2011). Randomised Controlled Feasibility Trial of an Evidence-Informed Behavioural Intervention for Obese Adults with Additional Risk Factors. PLoS ONE 6(8): e23040. doi:10.1371/journal.pone.0023040. 
Solomon, M., Ono, M., Timmer, S., \& Goodlin-Jones, B. (2008). A pilot study of the effectiveness of Parent Child Interaction Therapy (PCIT) for children on the autism spectrum. Journal of Autism and Developmental Disorders, 38, 1767-1776.

Stambaugh, L. F., Mustillo, S. A., Burns, B. J., Stephens, R. L., Baxter, B., Edwards, D., DeKraai, M. (2007). Outcomes from wraparound and multisystemic therapy in a center for mental health services system-of-care demonstration site. Journal of Emotional and Behavioral Disorders, 15, 143-155.

Stokes, J. (2014). Effectiveness of community-delivered parent-child interaction therapy compared to treatment as usual (Order No. 3637645). Available from Dissertations \& Theses @ West Virginia University; ProQuest Dissertations \& Theses Full Text. (1617955786). Retrieved from http://search.proquest.com/docview/1617955786?accountid=2837.

Suter, J. C., \& Bruns, E. J. (2009). Effectiveness of the wraparound process for children with emotional and behavioral disorders: A meta-analysis. Clinical Child and Family Psychology Review, 12, $336-351$.

Tiano, J.D., \& McNeil, C.B. (2005). Training Head Start teachers in behavior management using Parent-Child Interaction Therapy: A preliminary investigation. Journal of Early and Intensive Behavior Intervention, 3(2), 220-233.

Timmer, S. G., Ho, L. K. L., Urquiza, A. J., Zebell, N. M., Fernandez y Gracia, E., \& Boys, D. (2011). The effectiveness of parent-child interaction therapy with depressive mothers: The changing relationship as the agent of individual change. Child Psychiatry and Human Development, 42, 406-423.

Thomas, R. \& Zimmer-Gembeck (2007). Behavioral Outcomes of Parent-Child Interaction Therapy and Triple P-Positive Parenting Program: A Review and Meta-Analysis. Journal of Abnormal 
Child Psychology, 35, 475-495.

Trepka, C., Rees, A., Shapiro, D. A., Hardy, G. E., \& Barkham, M. (2004). Therapist competence and outcome of cognitive therapy for depression. Cognitive Therapy and Research, 28(2), 143-157. doi:10.1023/b:cotr.0000021536.39173.66

Lowry, R. (2001-2015). Two correlation coefficients. Retrieved April 11, 2016, from http://vassarstats.net/rdiff.html

Understanding "wraparound" services for children in healthchoices (2011). Retrieved from http://www.phlp.org/wp-content/uploads/2011/11/Guide-to-Understanding-WraparoundServices-Oct-20111.pdf.

United States Department of Health and Human Services (2005). Cooperative Agreements for the Comprehensive Community Mental Health Services for Children and Their Families Program (SM-05-010). Retrieved September 2, 2005, from http://www.systemsofcare.samhsa.gov.

VanDenBerg, J. (2008). Reflecting on wraparound: Inspirations, innovations, and future directions. In E. J. Bruns \& J. S. Walker (Eds.), The resource guide to wraparound. Portland, OR: National Wraparound Initiative, Research and Training Center for Family Support and Children's Mental Health.

VanDenBerg, J., Bruns, E. J., \& Burchard, J. (2008). History of the wraparound process. In E. J. Bruns \& J. S. Walker (Eds.), The resource guide to wraparound. Portland, OR: National Wraparound Initiative, Research and Training Center for Family Support and Children's Mental Health.

Wagner, S. M. \& McNeil, C. B. (2008). Parent-child interaction therapy for ADHD: A conceptual overview and critical literature review, Child and Family Behavior Therapy, 30, 231-256.

Walker, J. S. (2008). How, and why, does wraparound work: A theory of change. In E. J. Bruns \& J. S. Walker (Eds.), The resource guide to wraparound. Portland, OR: National Wraparound 
Initiative, Research and Training Center for Family Support and Children's Mental Health.

Walker, J.S., Bruns, E.J., Conlan, L. \& LaForce, C. (2011). The national wraparound initiative: A community of practice approach to building knowledge in the field of children's mental health. Best Practices in Mental Health, 7(1), 26-46.

Ware, L. M., McNeil, C. B., Masse, J., \& Stevens, S. (2008). Efficacy of in-home parent-child interaction therapy. Child \& Family Behavior Therapy, 30(2), 99-126.

Washington state institute for public policy (2015). Retrieved http://www.wsipp.wa.gov/BenefitCost.

Webb, C. A., Derubeis, R. J., \& Barber, J. P. (2010). Therapist adherence/competence and treatment outcome: A meta-analytic review. Journal of Consulting and Clinical Psychology, 78(2), 200211. doi:10.1037/a0018912

Webster-Stratton, C., \& Hammond, M. (1997). Treating children with early-onset conduct problems: A comparison of child and parent training interventions. Journal of Consulting and Clinical Psychology, 65, 93-109.

Weisz, J. R. (2000). Agenda for child and adolescent psychotherapy research: On the need to put science into practice. Archives of General Psychiatry, 57, 837-838.

Weisz, J. R., Sandler, I. N., Durlak, J. A., \& Anton, B. S. (2006). A proposal to unite two different worlds of children's mental health. American Psychologist, 61, 644-645.

Weisz, J. R., Weiss, B., Han, S., Granger, D. A., \& Morton, T. (1995). Effects of psychotherapy with children and adolescents revisited: A meta-analysis of treatment outcome studies. Psychological Bulletin, 117, 450-468.

Werba, B., Eyberg, S.M., Boggs, S.R., \& Algina, J. (2006). Predicting Outcome in Parent-Child Interaction Therapy: Success and Attrition. Behavior Modification, 30, 618-646. 
Winters, N.C., \& Metz, W.P. (2009). The Wraparound Approach in Systems of Care. Psychiatric Clinics of North America, 32, 135-151.

Wraparound Milwaukee. (2013). 2013 Year End Report. Retrieved from http://wraparoundmke.com/wp-content/uploads/2015/05/WMAnnualReport_2013.pdf. 
Table 1.

Study Enrollment, Assignment, and Retention Between Groups

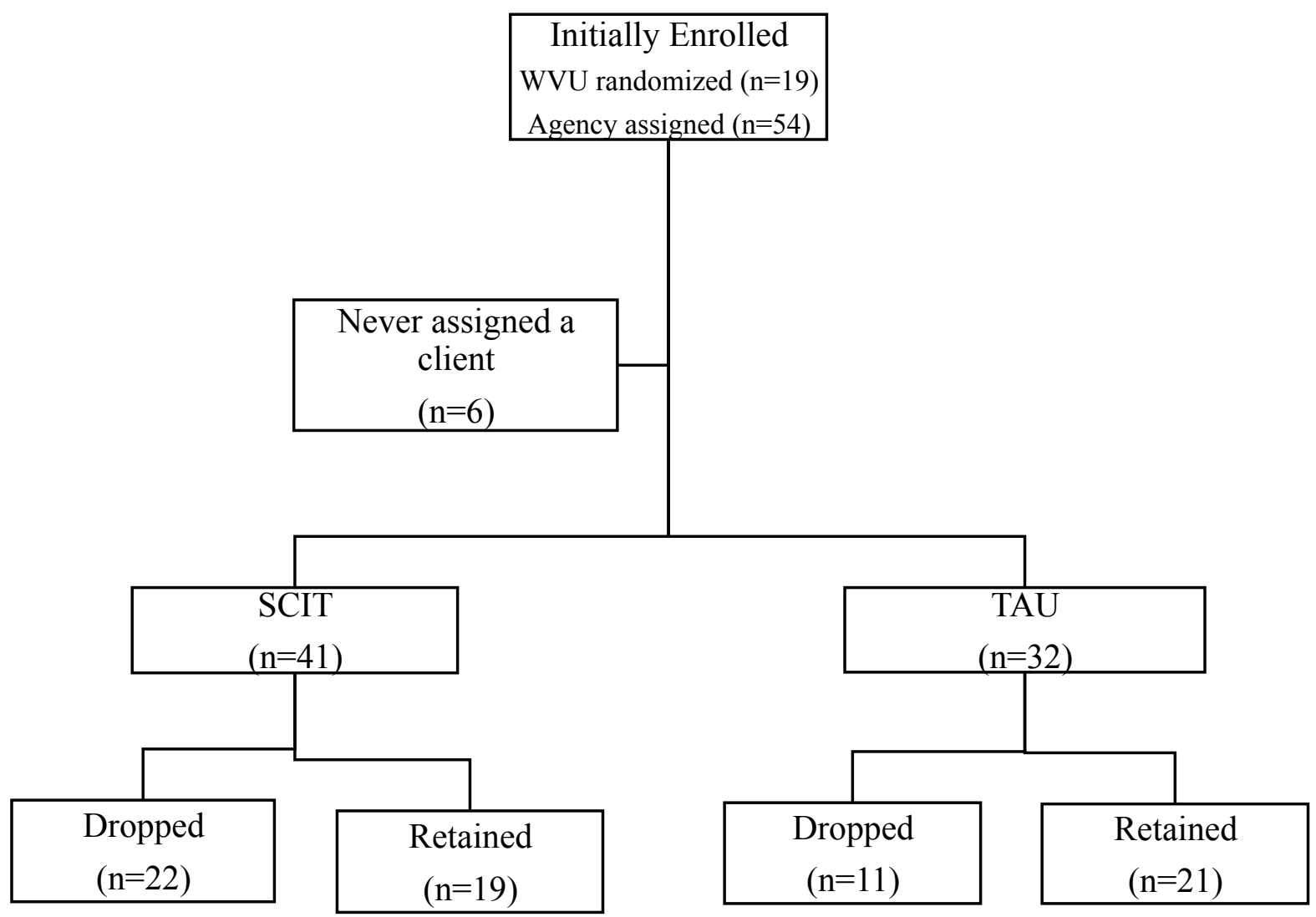


Table 2 .

Therapist Combined Group Means and Standard Deviations at Day One Workshop

\begin{tabular}{lc}
\hline \multicolumn{1}{c}{ Variable } & $M(S D)$ \\
\hline Sex & $84.9 \%$ Female \\
Race & $87.7 \%$ Caucasian \\
Education & $76.71 \%$ Bachelor's \\
Months in current position & $39.34(58.32)$ \\
Months as a therapist & $45.15(57.01)$ \\
Number of child clients total & $12.49(16.92)$ \\
Number of child clients currently & $3.23(1.86)$ \\
Total time working with client & $8.21(6.79)$ \\
Hours per week with client & $8.35(5.86)$ \\
Hours of previous training & $13.72(18.13)$ \\
Knowledge/Training in PCIT & $7.14 \%$ \\
Number of workshops attended & $2.26(.85)$ \\
\hline
\end{tabular}


Table 3.

Therapist Between Group Demographic Variables, Chi-Square, and Independent Samples T-Test Analyses at Workshop One

\begin{tabular}{|c|c|c|c|c|c|}
\hline & $\begin{array}{c}\text { SCIT } \\
M(S D)\end{array}$ & $\begin{array}{c}\text { TAU } \\
M(S D)\end{array}$ & $X^{2}$ & $t$ & $p$ \\
\hline \multirow[t]{2}{*}{ Therapist sex } & $87.80 \%$ & $81.25 \%$ & .60 & & .52 \\
\hline & Female & Female & & & \\
\hline \multirow[t]{2}{*}{ Therapist race } & $90.2 \%$ & $84.4 \%$ & .57 & & .49 \\
\hline & Caucasian & Caucasian & & & \\
\hline Therapist education & $85.4 \%$ & $65.6 \%$ & & -.95 & .35 \\
\hline $\begin{array}{l}\text { Hours of previous } \\
\text { training }\end{array}$ & $\begin{array}{l}\text { Bachelors } \\
8.70(7.20)\end{array}$ & $\begin{array}{c}\text { Bachelors } \\
19.83(24.74)\end{array}$ & & -2.27 & $.03^{*}$ \\
\hline $\begin{array}{l}\text { Previous PCIT } \\
\text { training }\end{array}$ & $7.9 \%$ & $6.3 \%$ & .71 & & 1.0 \\
\hline Months as a therapist & $46.66(60.94)$ & $43.22(52.45)$ & & .25 & .80 \\
\hline $\begin{array}{l}\text { Months in current } \\
\text { position }\end{array}$ & $42.45(58.32)$ & $35.32(39.87)$ & & .58 & .56 \\
\hline Total time with client & $8.6(7.74)$ & $7.75(5.52)$ & & .52 & .60 \\
\hline $\begin{array}{l}\text { Hours per week with } \\
\text { client }\end{array}$ & $7.51(5.62)$ & $9.42(6.07)$ & & .26 & .79 \\
\hline Total child clients & $11.60(11.68)$ & $13.59(21.95)$ & & -.49 & .62 \\
\hline $\begin{array}{l}\text { Number of child } \\
\text { clients currently }\end{array}$ & $3.34(1.85)$ & $3.09(1.89)$ & & .56 & .58 \\
\hline $\begin{array}{l}\text { Number of } \\
\text { workshops attended }\end{array}$ & $2.15(.82)$ & $2.41(.87)$ & -1.30 & & .20 \\
\hline
\end{tabular}


Table 4.

Child Combined Group Means and Standard Deviations at Day One Workshop

\begin{tabular}{lc}
\hline Variable & $M(S D)$ \\
\hline Sex & $76.1 \%$ Male \\
Race & $85.9 \%$ \\
& Caucasian \\
Age (in years) & $5.44(1.83)$ \\
Autism diagnosis & $60.27 \%$ \\
Disruptive behavior diagnosis & $50.68 \%$ \\
Time in mental health services (in months) & $26.59(22.06)$ \\
Current CPS & $2.7 \%$ \\
Past CPS & $16.4 \%$ \\
\hline
\end{tabular}


Table 5.

Child between Group Demographic Variables, Chi-Square, and Independent Samples T-Test Analyses at Workshop One

\begin{tabular}{lccccc}
\hline & SCIT & TAU & & & \\
& $M(S D)$ & $M(S D)$ & $X^{2}$ & $t$ & $p$ \\
\hline Child sex & $69.2 \%$ Male & $84.4 \%$ Male & 2.21 & & .14 \\
Child race & $92.3 \%$ & $78.1 \%$ & 2.92 & & .17 \\
& Caucasian & Caucasian & & & \\
Child age & $5.56(2.01)$ & $5.28(1.59)$ & & .64 & .52 \\
Child time in mental & $27.41(24.02)$ & $25.61(19.90)$ & & .31 & .76 \\
health services & & & & & \\
(months) & $12.2 \%$ & $19.35 \%$ & .70 & & .51 \\
Child past CPS & $2.4 \%$ & $3.1 \%$ & .03 & & 1.0 \\
Child current CPS & $56.1 \%$ & $65.63 \%$ & .68 & & .41 \\
Child mental health & Autism & Autism & & & .73 \\
diagnosis & $41.46 \%$ DBD & $37.5 \%$ DBD & .118 & & .78 \\
\end{tabular}


Table 6.

Correlational Matrix Comparing Workshop Two to Three, Parent Reported ECBI Difference

\begin{tabular}{lccccccc}
\hline Variable & 1 & 2 & 3 & 4 & 5 & 6 & 7 \\
& & & & & & & \\
\hline 1. ECBI difference score $(N=15)$ & - & & & & & & \\
2. Mastery percentage $(N=41)$ & .26 & - & & & & & \\
3. Time with client $(N=27)$ & -.08 & .14 & - & & & & \\
4. Hours/wk with client $(N=27)$ & -.37 & -.23 & -.01 & - & & \\
5. Total SCIT sessions $(N=20)$ & -.11 & .14 & -.10 & -.30 & - & \\
6. Total SCIT hours $(N=19)$ & -.03 & -.23 & -.24 & .20 & .11 & - & \\
7. Percentage of SCIT & .07 & -.37 & -.34 & .02 & .27 & $.80^{* *}$ & - \\
treatment $(N=19)$ & & & & & & & \\
\hline
\end{tabular}

Scores to SCIT Therapist Fidelity and Dose Variables

Note: $*=p<.05, * *=p<.01$ 
Table 7.

ECBI - Parent Perspective-Workshops Two to Three

\begin{tabular}{lccccc}
\hline ID Number & Group & Workshop 1 & Workshop 2 & Workshop 3 & $\begin{array}{c}\text { Average } \\
\text { Change } \\
\text { Score }\end{array}$ \\
\hline 7 & & & & -23 \\
19 & SCIT & 165 & 106 & 119 & -21.5 \\
28 & SCIT & 159 & 168.5 & 116 & 6 \\
36 & SCIT & 158 & 165 & 170 & -8.5 \\
81 & SCIT & 168 & 189 & 151 & -22.5 \\
82 & SCIT & 183 & 199 & 138 & 16.5 \\
83 & SCIT & 139 & 145 & 172 & -17 \\
85 & SCIT & 146 & 163 & 112 & -12 \\
40 & SCIT & 167 & 154 & 143 & -5 \\
42 & TAU & 169 & 149 & 159 & -0.5 \\
47 & TAU & 118 & 112 & 117 & -1.5 \\
48 & TAU & 97 & 73 & 94 & 8.5 \\
51 & TAU & 163 & 152 & 159 & 7.5 \\
52 & TAU & 120 & 149 & 137 & -18 \\
56 & TAU & 121 & 137.5 & 136 & 3.5 \\
57 & TAU & 143 & 123 & 107 & 12.5 \\
59 & TAU & 116 & 118.5 & 123 & -16.5 \\
60 & TAU & 116 & 165 & 141 & 9 \\
63 & TAU & 135 & 122 & 102 & 1 \\
89 & TAU & 138 & 133 & 156 & -31 \\
90 & TAU & 96 & 108 & 98 & -4 \\
92 & TAU & 212 & 200 & 150 & 105 \\
\hline
\end{tabular}


Table 8.

ECBI Means, Standard Deviations, F ratio, and p values across Workshops One, Two, and Three

\begin{tabular}{|c|c|c|c|c|c|c|c|c|}
\hline & $\begin{array}{c}\text { Workshop } 1 \\
M(S D)\end{array}$ & $\begin{array}{c}\text { Workshop } 2 \\
M(S D)\end{array}$ & $\begin{array}{c}\text { Workshop } 3 \\
M(S D)\end{array}$ & $\begin{array}{c}* \text { Change } \\
\text { Score } \\
M(S D)\end{array}$ & $\begin{array}{c}* * \text { Effect Size: } \\
\text { Cohen's D }\end{array}$ & $\begin{array}{c}\text { **Effect Size } \\
\text { (Cohen's D, } \\
\text { Partial Eta } \\
\text { Squared) }\end{array}$ & $F$ - Ratio & $p$ \\
\hline $\begin{array}{l}\text { Parent } \\
\text { ECBI } \\
(\mathrm{SCIT})\end{array}$ & $\begin{array}{l}160.63 \\
(13.66)\end{array}$ & $\begin{array}{c}161.1875 \\
(28.37)\end{array}$ & $\begin{array}{l}140.13 \\
(23.48)\end{array}$ & $\begin{array}{l}-10.25 \\
(14.49)\end{array}$ & -.93 (large) & $\begin{array}{c}-.49 \text { (medium); } \\
\text { (medium) }\end{array}$ & 1.19 & .33 \\
\hline $\begin{array}{l}\text { Parent } \\
\text { ECBI } \\
(\mathrm{TAU})\end{array}$ & $\begin{array}{l}132.64 \\
(31.31)\end{array}$ & $\begin{array}{l}131.79 \\
(30.86)\end{array}$ & $\begin{array}{l}127.42 \\
(23.77)\end{array}$ & $-2.61(12.05)$ & -.01 (no effect) & & & \\
\hline $\begin{array}{l}\text { Therapist } \\
\text { ECBI } \\
\text { (SCIT) }\end{array}$ & $\begin{array}{l}149.63 \\
(29.20)\end{array}$ & $155.5(20.16)$ & $\begin{array}{l}123.125 \\
(33.38)\end{array}$ & $\begin{array}{l}-13.25 \\
(15.06)\end{array}$ & -1.003 (large) & $\begin{array}{c}-.537 \text { (medium) } \\
.12 \text { (medium) }\end{array}$ & 2.23 & .124 \\
\hline $\begin{array}{l}\text { Therapist } \\
\text { ECBI } \\
\text { (TAU) }\end{array}$ & $\begin{array}{l}142.24 \\
(26.46)\end{array}$ & $\begin{array}{l}139.57 \\
(28.19)\end{array}$ & $\begin{array}{l}125.76 \\
(24.05)\end{array}$ & $-8.24(10.37)$ & -.588 (medium) & & & \\
\hline
\end{tabular}

Note: Scores are based on complete data sets. ${ }^{*}$ Change scores are calculated by averaging the difference scores between workshops three and two and workshops two and one. ** The average of workshop 1 and 2 means and standard deviations were used to determine pretest effect sizes. Effect size scores were calculated using Lenhard and Lenhard (2016). 
Table 9.

ECBI Scores - Parent Perspective, Workshops Two to Three

\begin{tabular}{|c|c|c|c|c|}
\hline ID & Group & Workshop 2 & Workshop 3 & Change Score \\
\hline 1 & SCIT & 165 & 141 & -24 \\
\hline 4 & SCIT & 213 & 167 & -46 \\
\hline 5 & SCIT & 122 & 86 & -36 \\
\hline 7 & SCIT & 106 & 119 & 13 \\
\hline 8 & SCIT & 163 & 169 & 6 \\
\hline 14 & SCIT & 188 & 155 & -33 \\
\hline 16 & SCIT & 101 & 78 & -23 \\
\hline 19 & SCIT & 168.5 & 116 & -52.5 \\
\hline 21 & SCIT & 196 & 101 & -95 \\
\hline 27 & SCIT & 107 & 107 & 0 \\
\hline 28 & SCIT & 165 & 170 & 5 \\
\hline 33 & SCIT & 172 & 149 & -23 \\
\hline 36 & SCIT & 189 & 151 & -38 \\
\hline 81 & SCIT & 199 & 138 & -61 \\
\hline 82 & SCIT & 145 & 172 & 27 \\
\hline 83 & SCIT & 163 & 112 & -51 \\
\hline 85 & SCIT & 154 & 143 & -11 \\
\hline 40 & TAU & 149 & 159 & 10 \\
\hline 41 & TAU & 183 & 158 & -25 \\
\hline 42 & TAU & 112 & 117 & 5 \\
\hline 47 & TAU & 73 & 94 & 21 \\
\hline 48 & TAU & 152 & 159 & 7 \\
\hline 50 & TAU & 216 & 182 & -34 \\
\hline 51 & TAU & 149 & 137 & -12 \\
\hline 52 & TAU & 137.5 & 136 & -1.5 \\
\hline 54 & TAU & 173 & 177 & 4 \\
\hline 56 & TAU & 123 & 107 & -16 \\
\hline 57 & TAU & 118.5 & 123 & 4.5 \\
\hline 59 & TAU & 165 & 141 & -24 \\
\hline 60 & TAU & 122 & 102 & -20 \\
\hline 63 & TAU & 133 & 156 & 23 \\
\hline 64 & TAU & 120 & 119 & -1 \\
\hline 89 & TAU & 108 & 98 & -10 \\
\hline 90 & TAU & 200 & 150 & -50 \\
\hline 92 & TAU & 103 & 105 & 2 \\
\hline
\end{tabular}


Table 10.

ECBI Means, Standard Deviations, F ratio, and p values across Workshops Two and Three

\begin{tabular}{|c|c|c|c|c|c|c|c|}
\hline & $\begin{array}{c}\text { Workshop } 2 \\
M(S D)\end{array}$ & $\begin{array}{c}\text { Workshop } 3 \\
M(S D)\end{array}$ & $\begin{array}{c}* \text { Change } \\
\text { Score } \\
M(S D)\end{array}$ & $\begin{array}{l}* * \text { Effect Size: } \\
\text { Cohen's D }\end{array}$ & $\begin{array}{c}\text { **Effect Size } \\
\text { (Cohen's D, } \\
\text { Partial Eta } \\
\text { Squared) }\end{array}$ & $F$ - Ratio & $p$ \\
\hline $\begin{array}{l}\text { Parent } \\
\text { ECBI } \\
(\mathrm{SCIT})\end{array}$ & $\begin{array}{l}159.79 \\
(33.95)\end{array}$ & $\begin{array}{l}133.77 \\
(30.05)\end{array}$ & $\begin{array}{l}-26.03 \\
(30.87)\end{array}$ & -.81 (large) & $\begin{array}{c}-.56 \text { (medium); } \\
\text { (large) }\end{array}$ & 5.14 & $.03 *$ \\
\hline $\begin{array}{l}\text { Parent } \\
\text { ECBI } \\
(\mathrm{TAU})\end{array}$ & $\begin{array}{l}140.94 \\
(36.32)\end{array}$ & $\begin{array}{l}134.44 \\
(27.55)\end{array}$ & $-6.5(19.07)$ & -.202 (no effect) & & & \\
\hline $\begin{array}{l}\text { Therapist } \\
\text { ECBI } \\
\text { (SCIT) }\end{array}$ & $156.5(18.68)$ & $126.39(33.14)$ & $\begin{array}{l}-30.11 \\
(35.02)\end{array}$ & -1.09 (large) & $\begin{array}{c}-.67 \text { (medium); } .09 \\
\text { (medium) }\end{array}$ & 3.69 & .06 \\
\hline $\begin{array}{l}\text { Therapist } \\
\text { ECBI } \\
(\mathrm{TAU})\end{array}$ & $\begin{array}{l}139.57 \\
(28.19)\end{array}$ & $\begin{array}{l}125.76 \\
(24.05)\end{array}$ & $\begin{array}{l}-13.81 \\
(15.67)\end{array}$ & -.53 (medium) & & & \\
\hline
\end{tabular}

Note: Scores are based on complete data sets. ${ }^{*}$ Change scores are calculated by averaging the difference scores between workshops three and two and workshops two and one. Effect size scores were calculated using Lenhard and Lenhard (2016). 
Table 11.

Eyberg Child Behavior Inventory Scores - Therapist Perspective, Workshops One, Two and Three

\begin{tabular}{|c|c|c|c|c|c|}
\hline ID Number & Group & Workshop 1 & Workshop 2 & Workshop 3 & $\begin{array}{c}\text { Average } \\
\text { Change } \\
\text { Score }\end{array}$ \\
\hline 1 & SCIT & 150 & 137 & 132 & -9 \\
\hline 5 & SCIT & 141 & 142 & 81 & -30 \\
\hline 7 & SCIT & 157 & 176 & 134 & -11.5 \\
\hline 8 & SCIT & 154 & 157 & 170 & 8 \\
\hline 14 & SCIT & 93 & 143 & 87 & -3 \\
\hline 16 & SCIT & 142 & 150 & 136 & -3 \\
\hline 19 & SCIT & 173 & 162 & 97 & -38 \\
\hline 23 & SCIT & 105 & 145 & 54 & -25.5 \\
\hline 25 & SCIT & 169 & 160 & 158 & -5.5 \\
\hline 27 & SCIT & 167 & 197 & 119 & -24 \\
\hline 28 & SCIT & 178 & 176 & 160 & -9 \\
\hline 33 & SCIT & 190 & 143 & 145 & -22.5 \\
\hline 36 & SCIT & 175 & 159 & 107 & -34 \\
\hline 37 & SCIT & 96 & 109 & 122 & 13 \\
\hline 81 & SCIT & 142 & 173 & 102 & -20 \\
\hline 82 & SCIT & 162 & 159 & 166 & 2 \\
\hline 40 & TAU & 109 & 120 & 132 & 11.5 \\
\hline 41 & TAU & 189 & 181 & 181 & -4 \\
\hline 42 & TAU & 153 & 134 & 125 & -14 \\
\hline 44 & TAU & 146 & 150 & 131 & -7.5 \\
\hline 47 & TAU & 106 & 107 & 83 & -11.5 \\
\hline 48 & TAU & 178 & 144 & 129 & -24.5 \\
\hline 50 & TAU & 164 & 147 & 125 & -19.5 \\
\hline 51 & TAU & 111 & 116 & 113 & 1 \\
\hline 52 & TAU & 113 & 89 & 93 & -10 \\
\hline 54 & TAU & 155 & 160 & 155 & 0 \\
\hline 55 & TAU & 127 & 152 & 128 & 0.5 \\
\hline 56 & TAU & 153 & 145 & 153 & 0 \\
\hline 57 & TAU & 120 & 97 & 97 & -11.5 \\
\hline 59 & TAU & 136 & 119 & 94 & -21 \\
\hline 60 & TAU & 140 & 131 & 124 & -8 \\
\hline 61 & TAU & 194 & 200 & 163 & -15.5 \\
\hline 63 & TAU & 149 & 130 & 126 & -11.5 \\
\hline 64 & TAU & 106 & 134 & 120 & 7 \\
\hline 89 & TAU & 131 & 175 & 132 & 0.5 \\
\hline 90 & TAU & 143 & 175 & 132 & -5.5 \\
\hline 92 & TAU & 164 & 125 & 105 & -29.5 \\
\hline
\end{tabular}


Table 12 .

Eyberg Child Behavior Inventory Scores - Therapist Perspective, Workshops Two to Three

\begin{tabular}{|c|c|c|c|c|}
\hline ID & Group & Workshop 2 & Workshop 3 & Change Score \\
\hline 1 & SCIT & 137 & 132 & -5 \\
\hline 4 & SCIT & 169 & 180 & 11 \\
\hline 5 & SCIT & 142 & 81 & -61 \\
\hline 7 & SCIT & 176 & 134 & -42 \\
\hline 8 & SCIT & 157 & 170 & 13 \\
\hline 14 & SCIT & 143 & 87 & -56 \\
\hline 16 & SCIT & 150 & 136 & -14 \\
\hline 19 & SCIT & 162 & 97 & -65 \\
\hline 21 & SCIT & 160 & 125 & -35 \\
\hline 23 & SCIT & 145 & 54 & -91 \\
\hline 25 & SCIT & 160 & 158 & -2 \\
\hline 27 & SCIT & 197 & 119 & -78 \\
\hline 28 & SCIT & 176 & 160 & -16 \\
\hline 33 & SCIT & 143 & 145 & 2 \\
\hline 36 & SCIT & 159 & 107 & -52 \\
\hline 37 & SCIT & 109 & 122 & 13 \\
\hline 81 & SCIT & 173 & 102 & -71 \\
\hline 82 & SCIT & 159 & 166 & 7 \\
\hline 40 & TAU & 120 & 132 & 12 \\
\hline 41 & TAU & 181 & 181 & 0 \\
\hline 42 & TAU & 134 & 125 & -9 \\
\hline 44 & TAU & 150 & 131 & -19 \\
\hline 47 & TAU & 107 & 83 & -24 \\
\hline 48 & TAU & 144 & 129 & -15 \\
\hline 50 & TAU & 147 & 125 & -22 \\
\hline 51 & TAU & 116 & 113 & -3 \\
\hline 52 & TAU & 89 & 93 & 4 \\
\hline 54 & TAU & 160 & 155 & -5 \\
\hline 55 & TAU & 152 & 128 & -24 \\
\hline 56 & TAU & 145 & 153 & 8 \\
\hline 57 & TAU & 97 & 97 & 0 \\
\hline 59 & TAU & 119 & 94 & -25 \\
\hline 60 & TAU & 131 & 124 & -7 \\
\hline 61 & TAU & 200 & 163 & -37 \\
\hline 63 & TAU & 130 & 126 & -4 \\
\hline 64 & TAU & 134 & 120 & -14 \\
\hline 89 & TAU & 175 & 132 & -43 \\
\hline 90 & TAU & 175 & 132 & -43 \\
\hline 92 & TAU & 125 & 105 & -20 \\
\hline
\end{tabular}


Table 13.

Anticipated Cost of SCIT Training Materials in Community Settings

\begin{tabular}{ll}
\hline Training Material Item & Cost \\
\hline SCIT manual (x8) & $\$ 128.64$ \\
ECBI measures (x12) & $\$ 21.60$ \\
SCIT training kit (x1) & $\$ 56.64$ \\
SCIT toys (x8) & $\$ 138.24$ \\
Workshop food & $\$ 800$ \\
\hline
\end{tabular}

Note: Costs are based on calculations for eight therapists across four workshops. The SCIT training kit includes coding sheets, mastery stars, name tags, DPICS quizzes, pencils, laminated diagrams, and graduation certificates. Workshop food is calculated for eight therapists over a four-day SCIT therapist training and four-day SCIT supervisor training. 
Table 14.

SCIT and TAU Therapist and Client Attrition

\begin{tabular}{|c|c|c|c|c|c|c|c|c|}
\hline ID & Group & Workshop 1 & Workshop 2 & Workshop 3 & Status & Attrition Reason & $\begin{array}{c}\text { ECBI } \\
\text { Outcome } \\
\text { (Parent) }\end{array}$ & $\begin{array}{c}\text { ECBI } \\
\text { Outcome } \\
\text { (Therapist) }\end{array}$ \\
\hline 1 & SCIT & $\begin{array}{l}\text { Therapist } 1 \\
\text { Child } 1\end{array}$ & $\begin{array}{l}\text { Therapist } 1 \\
\text { Child } 1\end{array}$ & $\begin{array}{l}\text { Therapist } 1 \\
\text { Child } 1\end{array}$ & Complete & & $\downarrow$ & - \\
\hline 2 & SCIT & $\begin{array}{l}\text { Therapist } 2 \\
\text { Child n/a }\end{array}$ & & & Incomplete & $\begin{array}{l}\text { Therapist did not } \\
\text { have appropriate } \\
\text { client }\end{array}$ & $\mathrm{n} / \mathrm{a}$ & $\mathrm{n} / \mathrm{a}$ \\
\hline 3 & SCIT & $\begin{array}{l}\text { Therapist } 3 \\
\text { Child } 1\end{array}$ & & & Incomplete & $\begin{array}{l}\text { Therapist withdrew } \\
\text { from TSS position }\end{array}$ & $\mathrm{n} / \mathrm{a}$ & $\mathrm{n} / \mathrm{a}$ \\
\hline 4 & SCIT & $\begin{array}{l}\text { Therapist } 4 \\
\text { Child } 1\end{array}$ & $\begin{array}{l}\text { Therapist } 4 \\
\text { Child } 2\end{array}$ & $\begin{array}{l}\text { Therapist } 4 \\
\text { Child } 2\end{array}$ & Complete & & $\downarrow$ & $\mathrm{n} / \mathrm{a}$ \\
\hline 5 & SCIT & $\begin{array}{l}\text { Therapist } 5 \\
\text { Child } 1\end{array}$ & $\begin{array}{l}\text { Therapist } 5 \\
\text { Child } 1\end{array}$ & $\begin{array}{l}\text { Therapist } 5 \\
\text { Child } 1\end{array}$ & Complete & & $\downarrow$ & $\downarrow$ \\
\hline 6 & SCIT & $\begin{array}{l}\text { Therapist } 6 \\
\text { Child } 1\end{array}$ & $\begin{array}{l}\text { Therapist } 6 \\
\text { Child } 1\end{array}$ & & Incomplete & $\begin{array}{l}\text { Therapist stopped } \\
\text { responding }\end{array}$ & $\mathrm{n} / \mathrm{a}$ & $\mathrm{n} / \mathrm{a}$ \\
\hline 7 & SCIT & $\begin{array}{c}\text { Therapist } 7 \\
\text { Child } 1\end{array}$ & $\begin{array}{l}\text { Therapist } 7 \\
\text { Child } 1\end{array}$ & $\begin{array}{l}\text { Therapist } 7 \\
\text { Child } 1\end{array}$ & Complete & & $\mathrm{n} / \mathrm{a}$ & $\downarrow$ \\
\hline 8 & SCIT & $\begin{array}{c}\text { Therapist } 8 \\
\text { Child } 1\end{array}$ & $\begin{array}{c}\text { Therapist } 8 \\
\text { Child } 1\end{array}$ & $\begin{array}{l}\text { Therapist } 8 \\
\text { Child } 1\end{array}$ & Complete & & - & - \\
\hline 9 & SCIT & $\begin{array}{l}\text { Therapist } 9 \\
\text { Child n/a }\end{array}$ & & & Incomplete & $\begin{array}{l}\text { Therapist did not } \\
\text { have appropriate } \\
\text { client }\end{array}$ & $\mathrm{n} / \mathrm{a}$ & $\mathrm{n} / \mathrm{a}$ \\
\hline 10 & SCIT & $\begin{array}{c}\text { Therapist } \\
10 \\
\text { Child } 1 \\
\text { Child } 2\end{array}$ & $\begin{array}{l}\text { Therapist } 10 \\
\text { Child } 1 \\
\text { Child } 2\end{array}$ & & Incomplete & $\begin{array}{l}\text { Therapist stopped } \\
\text { responding }\end{array}$ & - & $\mathrm{n} / \mathrm{a}$ \\
\hline 11 & SCIT & $\begin{array}{c}\text { Therapist } \\
11 \\
\text { Child } 1\end{array}$ & $\begin{array}{l}\text { Therapist } 11 \\
\text { Child } 1\end{array}$ & & Incomplete & $\begin{array}{l}\text { Therapist stopped } \\
\text { responding }\end{array}$ & $\mathrm{n} / \mathrm{a}$ & $\mathrm{n} / \mathrm{a}$ \\
\hline 12 & SCIT & $\begin{array}{c}\text { Therapist } \\
12 \\
\text { Child } 1\end{array}$ & $\begin{array}{l}\text { Therapist } 12 \\
\text { Child } 1\end{array}$ & & Incomplete & $\begin{array}{l}\text { Therapist withdrew } \\
\text { from TSS position }\end{array}$ & $\mathrm{n} / \mathrm{a}$ & $\mathrm{n} / \mathrm{a}$ \\
\hline 13 & SCIT & $\begin{array}{c}\text { Therapist } \\
13 \\
\text { Child n/a }\end{array}$ & & & Incomplete & $\begin{array}{l}\text { Therapist did not } \\
\text { have appropriate } \\
\text { client }\end{array}$ & $\mathrm{n} / \mathrm{a}$ & $\mathrm{n} / \mathrm{a}$ \\
\hline 14 & SCIT & $\begin{array}{c}\text { Therapist } \\
14 \\
\text { Child } 1\end{array}$ & $\begin{array}{l}\text { Therapist } 14 \\
\text { Child } 1\end{array}$ & $\begin{array}{l}\text { Therapist } 14 \\
\text { Child } 1\end{array}$ & Complete & & $\downarrow$ & - \\
\hline 15 & SCIT & $\begin{array}{c}\text { Therapist } \\
15 \\
\text { Child } 1\end{array}$ & & & Incomplete & $\begin{array}{l}\text { Therapist withdrew } \\
\text { from TSS position }\end{array}$ & $\mathrm{n} / \mathrm{a}$ & $\mathrm{n} / \mathrm{a}$ \\
\hline 16 & SCIT & $\begin{array}{c}\text { Therapist } \\
16 \\
\text { Child } 1\end{array}$ & $\begin{array}{l}\text { Therapist } 16 \\
\text { Child } 1\end{array}$ & $\begin{array}{l}\text { Therapist } 16 \\
\text { Child } 1\end{array}$ & Complete & & $\downarrow$ & - \\
\hline
\end{tabular}




\begin{tabular}{|c|c|c|c|c|c|c|c|c|}
\hline 17 & SCIT & $\begin{array}{c}\text { Therapist } \\
17 \\
\text { Child } 1\end{array}$ & & & Incomplete & $\begin{array}{l}\text { Therapist withdrew } \\
\text { from TSS position }\end{array}$ & $\mathrm{n} / \mathrm{a}$ & $\mathrm{n} / \mathrm{a}$ \\
\hline 18 & SCIT & $\begin{array}{c}\text { Therapist } \\
18 \\
\text { Child n/a }\end{array}$ & $\begin{array}{c}\text { Therapist } 18 \\
\text { Child n/a }\end{array}$ & & Incomplete & & $\mathrm{n} / \mathrm{a}$ & $\mathrm{n} / \mathrm{a}$ \\
\hline 19 & SCIT & $\begin{array}{c}\text { Therapist } \\
19 \\
\text { Child } 1\end{array}$ & $\begin{array}{l}\text { Therapist } 19 \\
\text { Child } 1\end{array}$ & $\begin{array}{c}\text { Therapist } 19 \\
\text { Child } 1\end{array}$ & Complete & & $\downarrow$ & $\downarrow$ \\
\hline 20 & SCIT & $\begin{array}{l}\text { Therapist } \\
20 \\
\text { Child } 1\end{array}$ & & & Incomplete & $\begin{array}{l}\text { Therapist withdrew } \\
\text { from TSS position }\end{array}$ & $\mathrm{n} / \mathrm{a}$ & $\mathrm{n} / \mathrm{a}$ \\
\hline 21 & SCIT & $\begin{array}{l}\text { Therapist } \\
21 \\
\text { Child } 1\end{array}$ & $\begin{array}{l}\text { Therapist } 21 \\
\text { Child } 2\end{array}$ & $\begin{array}{l}\text { Therapist } 21 \\
\text { Child } 2\end{array}$ & Complete & & $\downarrow$ & $\mathrm{n} / \mathrm{a}$ \\
\hline 22 & SCIT & $\begin{array}{l}\text { Therapist } \\
22 \\
\text { Child n/a }\end{array}$ & & & Incomplete & $\begin{array}{l}\text { Therapist did not } \\
\text { have appropriate } \\
\text { client }\end{array}$ & $\mathrm{n} / \mathrm{a}$ & $\mathrm{n} / \mathrm{a}$ \\
\hline 23 & SCIT & $\begin{array}{c}\text { Therapist } \\
23 \\
\text { Child 1 } \\
\text { Child 2 }\end{array}$ & $\begin{array}{l}\text { Therapist } 23 \\
\text { Child } 1 \\
\text { Child } 2\end{array}$ & $\begin{array}{l}\text { Therapist } 23 \\
\text { Child } 1 \\
\text { Child } 2\end{array}$ & Complete & & $\downarrow$ & $\downarrow$ \\
\hline 25 & SCIT & $\begin{array}{l}\text { Therapist } \\
25 \\
\text { Child } 1\end{array}$ & $\begin{array}{l}\text { Therapist } 25 \\
\text { Child } 1\end{array}$ & $\begin{array}{l}\text { Therapist } 25 \\
\text { Child } 1\end{array}$ & Complete & & $\downarrow$ & - \\
\hline 26 & SCIT & $\begin{array}{l}\text { Therapist } \\
26 \\
\text { Child n/a }\end{array}$ & & & Incomplete & $\begin{array}{l}\text { Therapist did not } \\
\text { have appropriate } \\
\text { client }\end{array}$ & $\mathrm{n} / \mathrm{a}$ & $\mathrm{n} / \mathrm{a}$ \\
\hline 27 & SCIT & $\begin{array}{c}\text { Therapist } \\
27 \\
\text { Child } 1\end{array}$ & $\begin{array}{l}\text { Therapist } 27 \\
\text { Child } 1\end{array}$ & $\begin{array}{l}\text { Therapist } 27 \\
\text { Child } 1\end{array}$ & Complete & & - & $\downarrow$ \\
\hline 28 & SCIT & $\begin{array}{c}\text { Therapist } \\
28 \\
\text { Child } 1\end{array}$ & $\begin{array}{l}\text { Therapist } 28 \\
\text { Child } 1\end{array}$ & $\begin{array}{l}\text { Therapist } 28 \\
\text { Child } 1\end{array}$ & Complete & & - & - \\
\hline 30 & SCIT & $\begin{array}{c}\text { Therapist } \\
30 \\
\text { Child } 1\end{array}$ & & & Incomplete & $\begin{array}{l}\text { Therapist withdrew } \\
\text { from TSS position }\end{array}$ & $\mathrm{n} / \mathrm{a}$ & $\mathrm{n} / \mathrm{a}$ \\
\hline 31 & SCIT & $\begin{array}{c}\text { Therapist } \\
31 \\
\text { Child } 1\end{array}$ & & & Incomplete & $\begin{array}{l}\text { Therapist stopped } \\
\text { responding }\end{array}$ & $\mathrm{n} / \mathrm{a}$ & $\mathrm{n} / \mathrm{a}$ \\
\hline 32 & SCIT & $\begin{array}{c}\text { Therapist } \\
32 \\
\text { Child } 1\end{array}$ & & & Incomplete & $\begin{array}{l}\text { Therapist stopped } \\
\text { responding }\end{array}$ & $\mathrm{n} / \mathrm{a}$ & $\mathrm{n} / \mathrm{a}$ \\
\hline 33 & SCIT & $\begin{array}{l}\text { Therapist } \\
33 \\
\text { Child 1 } \\
\text { Therapist }\end{array}$ & $\begin{array}{l}\text { Therapist } 33 \\
\text { Child } 1\end{array}$ & $\begin{array}{c}\text { Therapist } 33 \\
\text { Child } 1\end{array}$ & Complete & & - & $\downarrow$ \\
\hline 34 & SCIT & $\begin{array}{c}34 \\
\text { Child } 1\end{array}$ & & & Incomplete & Therapist lost client & $\mathrm{n} / \mathrm{a}$ & $\mathrm{n} / \mathrm{a}$ \\
\hline 35 & SCIT & $\begin{array}{c}\text { Therapist } \\
35 \\
\text { Child } 1\end{array}$ & & & Incomplete & Therapist lost client & $\mathrm{n} / \mathrm{a}$ & $\mathrm{n} / \mathrm{a}$ \\
\hline 36 & SCIT & $\begin{array}{c}\text { Therapist } \\
36 \\
\text { Child } 1\end{array}$ & $\begin{array}{l}\text { Therapist } 36 \\
\text { Child } 1\end{array}$ & $\begin{array}{l}\text { Therapist } 36 \\
\text { Child } 1\end{array}$ & Complete & & $\downarrow$ & $\downarrow$ \\
\hline & SCIT & $\begin{array}{c}\text { Therapist } \\
37 \\
\text { Child } 1\end{array}$ & $\begin{array}{l}\text { Therapist } 37 \\
\text { Child } 1\end{array}$ & $\begin{array}{l}\text { Therapist } 37 \\
\text { Child } 1\end{array}$ & Complete & & - & $\downarrow$ \\
\hline
\end{tabular}




\begin{tabular}{|c|c|c|c|c|c|c|c|c|}
\hline 40 & TAU & $\begin{array}{c}\text { Therapist } \\
40 \\
\text { Child } 1\end{array}$ & $\begin{array}{l}\text { Therapist } 40 \\
\text { Child } 1\end{array}$ & $\begin{array}{c}\text { Therapist } 40 \\
\text { Child } 1\end{array}$ & Complete & & - & $\downarrow$ \\
\hline 41 & TAU & $\begin{array}{c}\text { Therapist } \\
41 \\
\text { Child } 1\end{array}$ & $\begin{array}{l}\text { Therapist } 41 \\
\text { Child } 1\end{array}$ & $\begin{array}{l}\text { Therapist } 41 \\
\text { Child } 1\end{array}$ & Complete & & - & 一 \\
\hline 42 & TAU & $\begin{array}{c}\text { Therapist } \\
42 \\
\text { Child } 1\end{array}$ & $\begin{array}{l}\text { Therapist } 42 \\
\text { Child } 1\end{array}$ & $\begin{array}{c}\text { Therapist } 42 \\
\text { Child } 1\end{array}$ & Complete & & - & $\downarrow$ \\
\hline 43 & TAU & $\begin{array}{c}\text { Therapist } \\
43 \\
\text { Child } 1\end{array}$ & $\begin{array}{l}\text { Therapist } 43 \\
\text { Child } 1\end{array}$ & $\begin{array}{c}\text { Therapist } 43 \\
\text { Child } 1\end{array}$ & Complete & & $\mathrm{n} / \mathrm{a}$ & - \\
\hline 44 & TAU & $\begin{array}{c}\text { Therapist } \\
44 \\
\text { Child } 1\end{array}$ & $\begin{array}{l}\text { Therapist } 44 \\
\text { Child } 1\end{array}$ & $\begin{array}{l}\text { Therapist } 44 \\
\text { Child } 1\end{array}$ & Complete & & $\mathrm{n} / \mathrm{a}$ & - \\
\hline 45 & TAU & $\begin{array}{c}\text { Therapist } \\
45 \\
\text { Child } 1\end{array}$ & $\begin{array}{l}\text { Therapist } 45 \\
\text { Child } 1\end{array}$ & $\begin{array}{l}\text { Therapist } 45 \\
\text { Child } 1\end{array}$ & Incomplete & Therapist lost client & $\mathrm{n} / \mathrm{a}$ & $\mathrm{n} / \mathrm{a}$ \\
\hline 46 & TAU & $\begin{array}{c}\text { Therapist } \\
46 \\
\text { Child } 1\end{array}$ & $\begin{array}{l}\text { Therapist } 46 \\
\text { Child } 1\end{array}$ & $\begin{array}{l}\text { Therapist } 46 \\
\text { Child } 1\end{array}$ & Incomplete & $\begin{array}{l}\text { Therapist stopped } \\
\text { responding }\end{array}$ & $\mathrm{n} / \mathrm{a}$ & $\mathrm{n} / \mathrm{a}$ \\
\hline 47 & TAU & $\begin{array}{c}\text { Therapist } \\
47 \\
\text { Child } 1\end{array}$ & $\begin{array}{l}\text { Therapist } 47 \\
\text { Child } 1\end{array}$ & $\begin{array}{l}\text { Therapist } 47 \\
\text { Child } 1\end{array}$ & Complete & & - & $\downarrow$ \\
\hline 48 & TAU & $\begin{array}{c}\text { Therapist } \\
48 \\
\text { Child } 1\end{array}$ & $\begin{array}{l}\text { Therapist } 48 \\
\text { Child } 1\end{array}$ & $\begin{array}{l}\text { Therapist } 48 \\
\text { Child } 1\end{array}$ & Complete & & - & $\downarrow$ \\
\hline 49 & TAU & $\begin{array}{c}\text { Therapist } \\
49 \\
\text { Child } 1\end{array}$ & & & Incomplete & $\begin{array}{l}\text { Therapist withdrew } \\
\text { from TSS position }\end{array}$ & $\mathrm{n} / \mathrm{a}$ & $\mathrm{n} / \mathrm{a}$ \\
\hline 50 & TAU & $\begin{array}{c}\text { Therapist } \\
50 \\
\text { Child } 1\end{array}$ & $\begin{array}{l}\text { Therapist } 50 \\
\text { Child } 1\end{array}$ & $\begin{array}{l}\text { Therapist } 50 \\
\text { Child } 1\end{array}$ & Complete & & $\downarrow$ & $\downarrow$ \\
\hline 51 & TAU & $\begin{array}{c}\text { Therapist } \\
51 \\
\text { Child } 1\end{array}$ & $\begin{array}{l}\text { Therapist } 51 \\
\text { Child } 1\end{array}$ & $\begin{array}{l}\text { Therapist } 51 \\
\text { Child } 1\end{array}$ & Complete & & - & - \\
\hline 52 & TAU & $\begin{array}{c}\text { Therapist } \\
52 \\
\text { Child } 1\end{array}$ & $\begin{array}{l}\text { Therapist } 52 \\
\text { Child } 1\end{array}$ & $\begin{array}{l}\text { Therapist } 52 \\
\text { Child } 1\end{array}$ & Complete & & - & $\downarrow$ \\
\hline 53 & TAU & $\begin{array}{c}\text { Therapist } \\
53 \\
\text { Child } 1\end{array}$ & $\begin{array}{l}\text { Therapist } 53 \\
\text { Child } 1\end{array}$ & $\begin{array}{l}\text { Therapist } 53 \\
\text { Child } 1\end{array}$ & Incomplete & & $\mathrm{n} / \mathrm{a}$ & $\mathrm{n} / \mathrm{a}$ \\
\hline 54 & TAU & $\begin{array}{c}\text { Therapist } \\
54 \\
\text { Child } 1\end{array}$ & $\begin{array}{l}\text { Therapist } 54 \\
\text { Child } 1\end{array}$ & $\begin{array}{l}\text { Therapist } 54 \\
\text { Child } 1\end{array}$ & Complete & & - & - \\
\hline 55 & TAU & $\begin{array}{c}\text { Therapist } \\
55 \\
\text { Child } 1\end{array}$ & $\begin{array}{l}\text { Therapist } 55 \\
\text { Child } 1\end{array}$ & $\begin{array}{l}\text { Therapist } 55 \\
\text { Child } 1\end{array}$ & Complete & & $\mathrm{n} / \mathrm{a}$ & - \\
\hline 56 & TAU & $\begin{array}{c}\text { Therapist } \\
56 \\
\text { Child } 1\end{array}$ & $\begin{array}{l}\text { Therapist } 56 \\
\text { Child } 1\end{array}$ & $\begin{array}{l}\text { Therapist } 56 \\
\text { Child } 1\end{array}$ & Complete & & $\downarrow$ & 一 \\
\hline 57 & TAU & $\begin{array}{c}\text { Therapist } \\
57 \\
\text { Child } 1\end{array}$ & $\begin{array}{l}\text { Therapist } 57 \\
\text { Child } 1\end{array}$ & $\begin{array}{l}\text { Therapist } 57 \\
\text { Child } 1\end{array}$ & Complete & & - & $\downarrow$ \\
\hline 58 & TAU & $\begin{array}{c}\text { Therapist } \\
58 \\
\text { Child } 1\end{array}$ & & & Incomplete & $\begin{array}{l}\text { Therapist withdrew } \\
\text { from TSS position }\end{array}$ & $\mathrm{n} / \mathrm{a}$ & $\mathrm{n} / \mathrm{a}$ \\
\hline 59 & TAU & $\begin{array}{c}\text { Therapist } \\
59 \\
\text { Child } 1\end{array}$ & $\begin{array}{l}\text { Therapist } 59 \\
\text { Child } 1\end{array}$ & $\begin{array}{c}\text { Therapist } 59 \\
\text { Child } 1\end{array}$ & Complete & & - & $\downarrow$ \\
\hline
\end{tabular}




\begin{tabular}{|c|c|c|c|c|c|c|c|c|}
\hline 60 & TAU & $\begin{array}{c}\text { Therapist } \\
60 \\
\text { Child } 1\end{array}$ & $\begin{array}{l}\text { Therapist } 60 \\
\text { Child } 1\end{array}$ & $\begin{array}{c}\text { Therapist } 60 \\
\text { Child } 1\end{array}$ & Complete & & $\downarrow$ & - \\
\hline 61 & TAU & $\begin{array}{c}\text { Therapist } \\
61 \\
\text { Child } 1\end{array}$ & $\begin{array}{l}\text { Therapist } 61 \\
\text { Child } 1\end{array}$ & $\begin{array}{c}\text { Therapist } 61 \\
\text { Child } 1\end{array}$ & Complete & & $\mathrm{n} / \mathrm{a}$ & $\downarrow$ \\
\hline 62 & TAU & $\begin{array}{c}\text { Therapist } \\
62 \\
\text { Child } 1\end{array}$ & & & Incomplete & $\begin{array}{l}\text { Therapist withdrew } \\
\text { from TSS position }\end{array}$ & $\mathrm{n} / \mathrm{a}$ & $\mathrm{n} / \mathrm{a}$ \\
\hline 63 & TAU & $\begin{array}{c}\text { Therapist } \\
63 \\
\text { Child } 1\end{array}$ & $\begin{array}{l}\text { Therapist } 63 \\
\text { Child } 1\end{array}$ & $\begin{array}{c}\text { Therapist } 63 \\
\text { Child } 1\end{array}$ & Complete & & $\uparrow$ & $\downarrow$ \\
\hline 64 & TAU & $\begin{array}{c}\text { Therapist } \\
64 \\
\text { Child } 1\end{array}$ & $\begin{array}{l}\text { Therapist } 64 \\
\text { Child } 1\end{array}$ & $\begin{array}{c}\text { Therapist } 64 \\
\text { Child } 1\end{array}$ & Complete & & - & - \\
\hline 65 & TAU & $\begin{array}{c}\text { Therapist } \\
65 \\
\text { Child } 1\end{array}$ & $\begin{array}{l}\text { Therapist } 65 \\
\text { Child } 1\end{array}$ & & Incomplete & $\begin{array}{l}\text { Therapist withdrew } \\
\text { from TSS position }\end{array}$ & $\mathrm{n} / \mathrm{a}$ & $\mathrm{n} / \mathrm{a}$ \\
\hline 80 & SCIT & $\begin{array}{c}\text { Therapist } \\
80 \\
\text { Child } 1\end{array}$ & $\begin{array}{l}\text { Therapist } 80 \\
\text { Child } 1\end{array}$ & $\begin{array}{c}\text { Therapist } 80 \\
\text { Child } 1\end{array}$ & Complete & & $\mathrm{n} / \mathrm{a}$ & $\mathrm{n} / \mathrm{a}$ \\
\hline 81 & SCIT & $\begin{array}{c}\text { Therapist } \\
81 \\
\text { Child } 1\end{array}$ & $\begin{array}{l}\text { Therapist } 81 \\
\text { Child } 1\end{array}$ & $\begin{array}{c}\text { Therapist } 81 \\
\text { Child } 1\end{array}$ & Complete & & $\downarrow$ & $\downarrow$ \\
\hline 82 & SCIT & $\begin{array}{c}\text { Therapist } \\
82 \\
\text { Child } 1\end{array}$ & $\begin{array}{l}\text { Therapist } 82 \\
\text { Child } 1\end{array}$ & $\begin{array}{c}\text { Therapist } 82 \\
\text { Child } 1\end{array}$ & Complete & & $\uparrow$ & - \\
\hline 83 & SCIT & $\begin{array}{c}\text { Therapist } \\
83 \\
\text { Child } 1\end{array}$ & $\begin{array}{l}\text { Therapist } 83 \\
\text { Child } 1\end{array}$ & & Incomplete & $\begin{array}{l}\text { Therapist withdrew } \\
\text { from TSS position }\end{array}$ & $\downarrow$ & $\mathrm{n} / \mathrm{a}$ \\
\hline 84 & SCIT & $\begin{array}{c}\text { Therapist } \\
84 \\
\text { Child } 1\end{array}$ & & & Incomplete & Therapist lost client & $\mathrm{n} / \mathrm{a}$ & $\mathrm{n} / \mathrm{a}$ \\
\hline 85 & SCIT & $\begin{array}{c}\text { Therapist } \\
85 \\
\text { Child } 1 \\
\text { Child } 2\end{array}$ & $\begin{array}{l}\text { Therapist } 85 \\
\text { Child } 1 \\
\text { Child } 2\end{array}$ & & Incomplete & $\begin{array}{l}\text { Therapist withdrew } \\
\text { from TSS position }\end{array}$ & - & $\mathrm{n} / \mathrm{a}$ \\
\hline 89 & TAU & $\begin{array}{c}\text { Therapist } \\
89 \\
\text { Child } 1\end{array}$ & $\begin{array}{l}\text { Therapist } 89 \\
\text { Child } 1\end{array}$ & $\begin{array}{c}\text { Therapist } 89 \\
\text { Child } 1\end{array}$ & Complete & & - & - \\
\hline 90 & TAU & $\begin{array}{c}\text { Therapist } \\
90 \\
\text { Child } 1\end{array}$ & $\begin{array}{l}\text { Therapist } 90 \\
\text { Child } 1\end{array}$ & $\begin{array}{c}\text { Therapist } 90 \\
\text { Child } 1\end{array}$ & Complete & & $\downarrow$ & - \\
\hline 91 & TAU & $\begin{array}{c}\text { Therapist } \\
91 \\
\text { Child } 1\end{array}$ & & & Incomplete & $\begin{array}{l}\text { Therapist withdrew } \\
\text { from TSS position }\end{array}$ & $\mathrm{n} / \mathrm{a}$ & $\mathrm{n} / \mathrm{a}$ \\
\hline 92 & TAU & $\begin{array}{c}\text { Therapist } \\
92 \\
\text { Child } 1\end{array}$ & $\begin{array}{l}\text { Therapist } 92 \\
\text { Child } 1\end{array}$ & $\begin{array}{c}\text { Therapist } 92 \\
\text { Child } 1\end{array}$ & Complete & & - & $\downarrow$ \\
\hline 93 & TAU & $\begin{array}{c}\text { Therapist } \\
93 \\
\text { Child } 1\end{array}$ & & & Incomplete & $\begin{array}{l}\text { Therapist stopped } \\
\text { responding }\end{array}$ & $\mathrm{n} / \mathrm{a}$ & $\mathrm{n} / \mathrm{a}$ \\
\hline 94 & TAU & $\begin{array}{c}\text { Therapist } \\
94 \\
\text { Child } 1\end{array}$ & & & Incomplete & $\begin{array}{l}\text { Therapist withdrew } \\
\text { from TSS position }\end{array}$ & $\mathrm{n} / \mathrm{a}$ & $\mathrm{n} / \mathrm{a}$ \\
\hline
\end{tabular}


Table 15.

SCIT Workshop Qualitative Feedback Response Themes

\begin{tabular}{|c|c|}
\hline Theme- Positive Feedback & Sample Responses \\
\hline \multirow[t]{5}{*}{ Workshop Structure } & "The hands on practice" \\
\hline & "The interactive role play. I $[\mathrm{t}]$ help[ed] the info sink in" \\
\hline & "The interactive participation, hands on training, and feedback" \\
\hline & "Role play experiences" \\
\hline & $\begin{array}{l}\text { "So much information, very thorough detailing. Hands-on interacting made it } \\
\text { more interesting" }\end{array}$ \\
\hline Learning New & "Informative regarding the interactions between the staff, parents, and child" \\
\hline \multirow[t]{3}{*}{ Skills/Information } & "Information will be effective with client" \\
\hline & $\begin{array}{l}\text { "The informative answers whe[n] asked and the obvious love of the } \\
\text { clinicians of this program" }\end{array}$ \\
\hline & "Learning better ways to incorporate families and handle different situations" \\
\hline \multirow[t]{4}{*}{ Workshop Format } & "It was very organized and I think it'll work perfectly for the kiddo who I \\
\hline & TSS for” \\
\hline & "Break-out sessions" \\
\hline & $\begin{array}{l}\text { "Questions and potential problems/concerns were addressed in timely } \\
\text { manner until clarified- nobody forgot questions while training continued, we } \\
\text { had time to write notes and organize our thoughts" }\end{array}$ \\
\hline \multirow[t]{3}{*}{ Skill Practice } & "The practice sessions and guidance through steps. Positive" \\
\hline & $\begin{array}{l}\text { "I liked practicing the skills so that I have confidence to use them. Best } \\
\text { training ever!!" }\end{array}$ \\
\hline & $\begin{array}{l}\text { "Interacting and practicing the skills we will be using while being guided } \\
\text { through it. All questions were answered" }\end{array}$ \\
\hline \multirow[t]{5}{*}{ Instructor Qualities } & $\begin{array}{l}\text { "The interaction and the amount instructor engaged us. The practice really } \\
\text { helps!" }\end{array}$ \\
\hline & "How comfortable everyone was made to feel" \\
\hline & "Everyone on team is knowledgeable. So impressed with your undergrads!!" \\
\hline & $\begin{array}{l}\text { "How positive and enthusiastic everyone was. The success stories were } \\
\text { inspiring" }\end{array}$ \\
\hline & $\begin{array}{l}\text { "Staff very friendly, informative, and helpful! Very energetic! Best workshop } \\
\text { I have attended!" }\end{array}$ \\
\hline Workshop Discussions & $\begin{array}{l}\text { "Hearing others experiences and the ability [to] talk about problems, } \\
\text { successes, etc" }\end{array}$ \\
\hline \multicolumn{2}{|l|}{ Theme- Improvement } \\
\hline Workshop Structure & $\begin{array}{l}\text { "Make it longer and build in more breaks" } \\
\text { "Teach over a 3-4 day period" }\end{array}$ \\
\hline \multirow[t]{2}{*}{ Setting } & "Better facilities and access" \\
\hline & $\begin{array}{l}\text { "I don't like the chairs in the first room. They hurt my back, hard to } \\
\text { concentrate" }\end{array}$ \\
\hline \multirow[t]{2}{*}{ Workshop Content } & $\begin{array}{l}\text { "Better clarification on how to teach parents before the exercise with the } \\
\text { students" }\end{array}$ \\
\hline & "Maybe go a little slower, but I'm aware that it's not very time permitting" \\
\hline
\end{tabular}


Table 16.

TAU Workshop Qualitative Feedback Response Themes

\begin{tabular}{|c|c|}
\hline Theme - Positive Feedback & Sample Responses \\
\hline \multirow[t]{4}{*}{ Workshop Structure } & "Hands-on, how positive everyone was" \\
\hline & "Interactive and collaborative" \\
\hline & "The different activities that kept us moving around" \\
\hline & $\begin{array}{l}\text { "Open atmosphere, appropriate materials and activities, professional } \\
\text { presentation" }\end{array}$ \\
\hline \multirow[t]{4}{*}{ Compassion Fatigue Material } & "Relaxation skills" \\
\hline & "Meditation" \\
\hline & $\begin{array}{l}\text { "Learning new coping techniques to deal with stress and sharing } \\
\text { concerns about TSS role" }\end{array}$ \\
\hline & $\begin{array}{l}\text { "Acquiring new information and tactics to prevent burnout. Also being } \\
\text { able to present ideas to decrease turnover within the company" }\end{array}$ \\
\hline Agency Comments & "Enjoyed that [the agency] is looking to change" \\
\hline \multirow[t]{5}{*}{ Discussion } & "Group discussion and games" \\
\hline & "Being able to talk to fellow co-workers about the job and experiences" \\
\hline & "Interesting questions asked, hearing feedback from other TSS" \\
\hline & "Opportunities to express our concerns" \\
\hline & "Small group questions and discussions" \\
\hline \multirow[t]{4}{*}{ Instructor Qualities } & "Open and friendly" \\
\hline & "Everyone was welcoming and helpful throughout the entire session" \\
\hline & $\begin{array}{l}\text { "Food, friendly people presenting the information. Kept interest and was } \\
\text { fun" }\end{array}$ \\
\hline & "Presented in a knowledgeable manner" \\
\hline \multirow[t]{2}{*}{ SCIT Review } & "Learning about SCIT" \\
\hline & "The overview of SCIT" \\
\hline \multicolumn{2}{|l|}{ Theme- Improvement } \\
\hline \multirow[t]{2}{*}{ Workshop Content } & "I would want to be in the group learning the SCIT" \\
\hline & "More SCIT therapy for compassion fatigue group" \\
\hline \multirow[t]{2}{*}{ Instructor Quality } & $\begin{array}{l}\text { "Talk with someone within the position to have a clearer understanding } \\
\text { and more realistic scenarios" }\end{array}$ \\
\hline & "Clarify and communication" \\
\hline \multirow[t]{2}{*}{ Training Opportunity } & $\begin{array}{l}\text { "Better collaboration with [the agency] office employees about the } \\
\text { training" }\end{array}$ \\
\hline & $\begin{array}{l}\text { "When told of this, expected more training on working with clients and } \\
\text { not on self-improvement" }\end{array}$ \\
\hline \multirow[t]{2}{*}{ Assessments Utilized } & "No role playing" \\
\hline & $\begin{array}{l}\text { "The scenarios with the undergraduate student, be more realistic to our } \\
\text { job" }\end{array}$ \\
\hline
\end{tabular}


Table 17.

SSOC Means, Standard Deviations, F ratio, and p values Across Workshops One, Two, and Three

\begin{tabular}{|c|c|c|c|c|c|c|c|}
\hline & $\begin{array}{c}\text { Workshop } 1 \\
M(S D)\end{array}$ & $\begin{array}{c}\text { Workshop } 2 \\
M(S D)\end{array}$ & $\begin{array}{c}\text { Workshop } 3 \\
M(S D)\end{array}$ & $\begin{array}{l}\text { *Effect Size: } \\
\text { Cohen's D }\end{array}$ & $\begin{array}{c}\text { *Effect Size } \\
\text { (Cohen's D, } \\
\text { Partial Eta } \\
\text { Squared) }\end{array}$ & $F$ - Ratio & $p$ \\
\hline SCIT & $72.16(10.42)$ & $75.53(9.82)$ & $81.11(11.03)$ & .686 (medium) & $\begin{array}{c}.571 \text { (medium) } \\
.25 \text { (large) }\end{array}$ & 6.12 & $.01 *$ \\
\hline TAU & $71.81(9.91)$ & $70.24(7.29)$ & $72.90(9.55)$ & .207 (small) & & & \\
\hline
\end{tabular}

Note: Scores are based on complete data sets. * The average of workshop 1 and 2 means and standard deviations were used to determine pretest effect sizes. Effect size scores were calculated using Lenhard and Lenhard (2016). 
Table 18.

TAU Discussion Themes.

Theme: BHRS Policies in Conflict with Expected Treatment Outcomes

Policy-Based

Limitations

Consumer Model
1. TSS primarily provides individual treatment but can't be alone with child

2. Limited to work with child, unable to teach caregivers skills leading to disconnect between treatment execution and transfer of skills to parents

3. TSS primary focus on assessment (tallying behaviors) versus intervention (modifying behaviors) $\rightarrow$ mixed messages regarding TSS as the agent of change

4. Minimal involvement/influence of TSS on creation of treatment plan but restricted to services based on treatment plan

5. Sleeping/napping child results in conclusion of services for the day (no billable hours for TSS)

6. Conceptualization/measurement of progress- data is difficult to collect due to unclear data sheets, unclear operationalization of behaviors, inconsistent data collection due to different data sheets resulting to invalid/ineffective data ("sometimes you have to count behaviors in your head" resulting in "skewed data")

- Lack of authority/control within family (TSS is providing treatment but family has ultimate say over which treatment is provided, how it is provided, and where it occurs)

- Breakdown in communication (who is in charge) leading to mixed messages between TSS and parent

- Lack of predictability for TSS and child client 


\author{
Theme: BHRS \\ Consistency \\ Role \\ Expectations/Enforcem \\ ent of Role
}

Lack of Parent Involvement/ Lack of Skill transfer

Inconsistent Contextual Service Factors

Lack of psychoeducation/specialized training regarding diagnostic conditions Supervision
- Parental misperception of therapist role. TSS viewed as babysitter, helper, friend to parent and consumer, teacher, mentor, therapist for consumer)

- Leads to disrespect for TSS by parents, misattributions of who is the agent of change, mixed messages for child

- School-based setting - teacher dependency upon TSS

- Lack of engagement/cooperation or over-engaged parent in treatment (e.g., present but not involved or try to take control)

- Breakdown in communication between agency expectations-parents expectations-therapist expectations

- Unpredictable TSS billable hours due to client cancellations

- Unpredictable client time to termination

- Environmental distractions (e.g., siblings, video games, $\mathrm{TV}$, noise level, pre-session activities) leading to interference with effective treatment

- Among therapist, families, siblings

- Little use of empirically-based treatment programs

- Variable physical presence/involvement of BSC with TSS

- Disconnect in communication when TSS provides treatment with client and BSC provides treatment with client

- Lack of accountability for effective supervision

- Lack of effective treatment skills provided in group supervision

- Lack of overall supervision time (individual/group)

\section{Theme: Impact upon Therapists/Families}

Lack of Client Progress

Therapist Burn Out/Turnover
- Results in change in child but no change in parent $\rightarrow$ change in child when TSS is present but no change when TSS is absent

- TSS learns not to invest more than families

- Lack of support/guidance from agency/supervisors on difficult cases

- Feeling overwhelmed/ineffective within job

- Tiring job- long day in treatment then must write notes

- Poor pay/reimbursement for gas 
- Lack of predictability

- No incentives (raises occur at end of year, not significant (.20 cents), no benefits for seniority)

TSS Suggestions for Improvement

- Lack of evaluations of TSS/management

- Ability to talk about cases with people informed about clients

- Evaluations for management

- Limiting therapist travel 
Table 19.

SCIT Therapist Feedback following SCIT Implementation

\begin{tabular}{|c|c|}
\hline Theme & Representative Responses \\
\hline Therapist Pretreatment & "Increased verbalization" \\
\hline \multirow[t]{10}{*}{ Desired Outcomes } & "Appropriate play skills and improved social interaction" \\
\hline & "Increased parent involvement/ transfer of skill to parent" \\
\hline & "Increased positive client behavior" \\
\hline & "Increased compliance" \\
\hline & "Positive parent-child relationship" \\
\hline & "More positive and effective way to communicate" \\
\hline & "Improved client confidence" \\
\hline & "Decreased aggression" \\
\hline & "Better therapist-client relationship" \\
\hline & "Parent ignoring some behaviors" \\
\hline \multirow[t]{16}{*}{ Client Changes } & "Increased eye contact" \\
\hline & "Requests to play with parents" \\
\hline & "Expresses needs and wants better" \\
\hline & "Appropriate play" \\
\hline & "Increased compliance" \\
\hline & "Improved communication and use of language" \\
\hline & "Increased attention to task" \\
\hline & "Improved manners" \\
\hline & "More gentle" \\
\hline & "Increased confidence" \\
\hline & "Client stays calm" \\
\hline & "More independent" \\
\hline & "Showing and naming toys" \\
\hline & Behaviors that continue to occur: \\
\hline & "Tantrums still occur" \\
\hline & "Some negative behaviors maintained" \\
\hline \multirow{12}{*}{$\begin{array}{l}\text { Therapist Perceptions of } \\
\text { Parent Changes }\end{array}$} & "Caregivers interact with client more positively and effectively" \\
\hline & "Caregivers actually enjoy playing with client" \\
\hline & "Caregiver is so much more involved" \\
\hline & "Better and more understanding relationship overall" \\
\hline & "Increased praise" \\
\hline & "Spend more time together" \\
\hline & "Caregiver showed more interest in playing with client" \\
\hline & "Caregivers more aware of positive behaviors" \\
\hline & "Parent has stopped running the show" \\
\hline & Interactions that continue to occur: \\
\hline & "Interactions still the same when therapist is not involved" \\
\hline & "Parents did not change" \\
\hline
\end{tabular}


Therapist Perceptions of Changes in Themselves

Barriers to SCIT Implementation

Suggestions for Improvement in SCIT program
"Skills in implementing and in overall interactions"

"Realized the parent-child relationship was important"

"Therapist more aware of negative talk"

"More aware of client's good behavior"

"Using PRIDE skills with everyone"

"Child/Parent health issues"

"Homework"

"Getting BSC on board"

"Control of environment"

"Getting caregivers on board"

"Family canceling BHRS services without notice"

"Parents allowing child to have the lead"

"BSC/parents/TSS not on same page"

"ADI (child always complied so couldn't show effective commands)"

"Getting child to focus and comply"

"A sheet listing various scenarios to help therapist better prepare when problems arise, tips for getting client to talk during ADI" "Different way to track homework (client lost homework sheets since there were so many)"

"Less of a staff-model more with mom"

"Have TSS/BSC explain CDI and ADI extensively before parent tries it"

"Alternative ways to do ADI with a client who aims to please and does anything that is asked"

"Parents need more thorough training in SCIT before starting treatment"

"Shorter time between trainings 1 and 2"

"Toys for older kids"

"CDI wasn't long enough"

"More training for BSC"

"Focus on CDI until is mastered and then move ADI" 
Figure 1. Parent CDI Skills Across SCIT Sessions (Median)

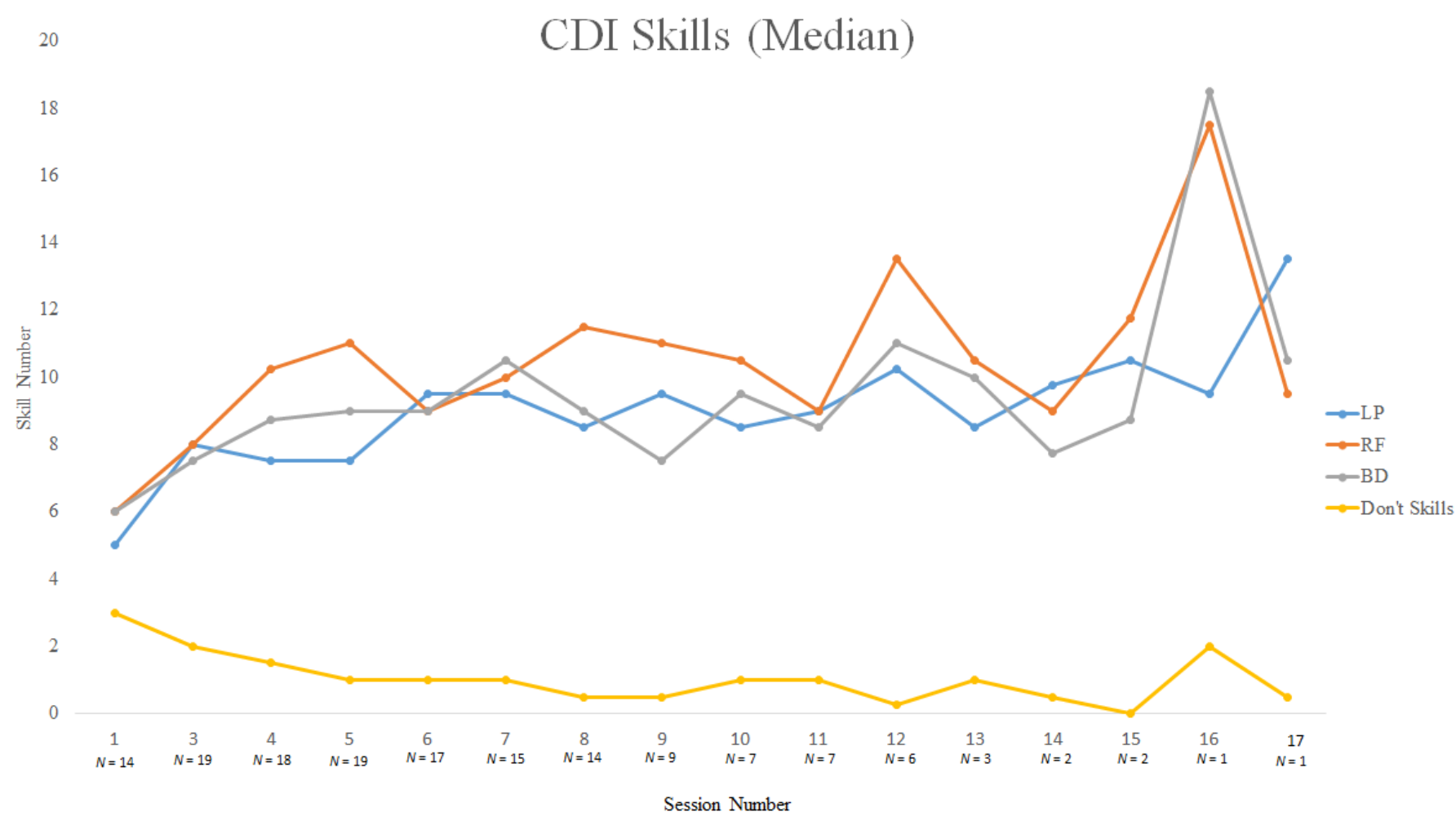


Figure 2. Parent ECBI Change throughout First, Second, and Third Workshops between the $\operatorname{SCIT}(N=8)$ and TAU $(N=14)$ Groups

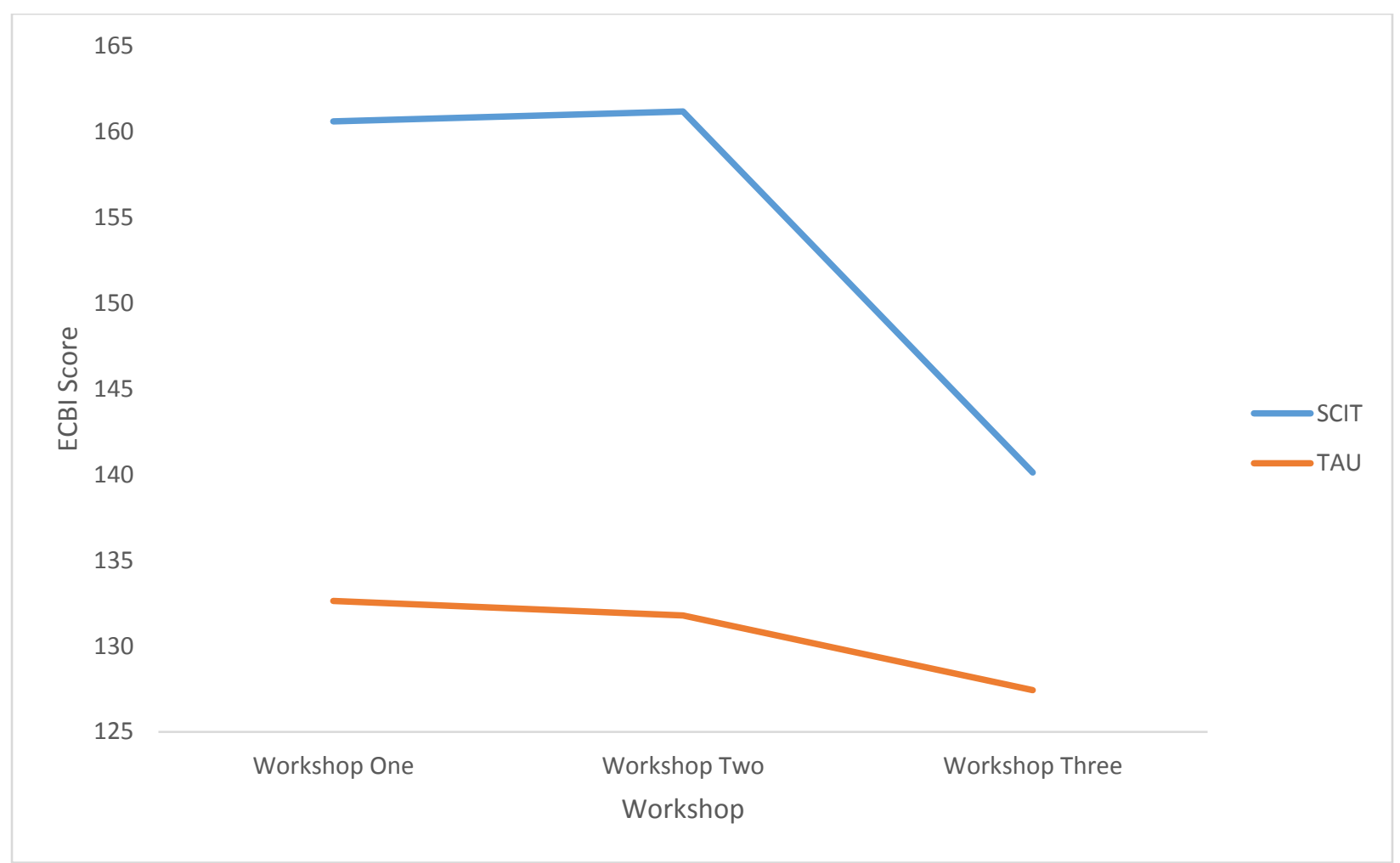


Figure 3. Parent ECBI Change throughout the Second and Third Workshops between the SCIT $(N=17)$ and $T A U(N=18)$ Groups

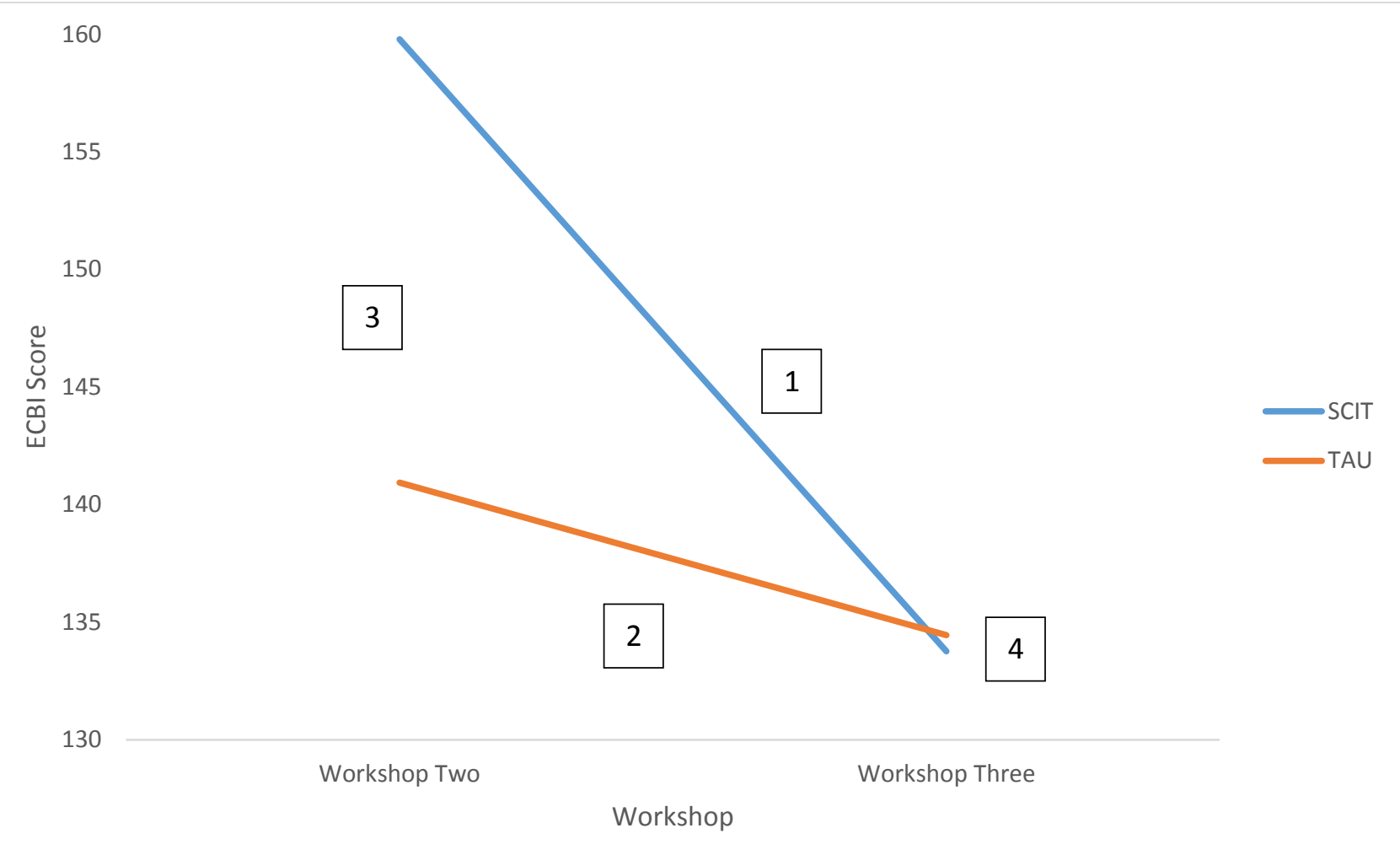

Note:

1. Post hoc comparisons using the Tukey HSD test indicated that the mean score for the SCIT group at workshop two $(M=159.79, S D=33.95)$ was significantly different from the mean score for the SCIT group at workshop three $(M=133.76, S D=30.05), p<.01$.

2. Post hoc comparisons using the Tukey HSD test indicated that the mean score for the TAU group at workshop two $(M=140.94, S D=36.32)$ was not significantly different from the mean score for the TAU group at workshop three $(M=134.44, S D=27.55), p>.05$.

3. Post hoc comparisons using the Tukey HSD test indicated that the mean score for the SCIT group at workshop two $(M=159.79, S D=33.95)$ was significantly different from the mean score for the TAU group at workshop two $(M=140.94, S D=36.32), p<.05$.

4. Post hoc comparisons using the Tukey HSD test indicated that the mean score for the SCIT group at workshop three $(M=133.76, S D=30.05)$ was not significantly different from the mean score for the TAU group at workshop three $(M=134.44, S D=27.55), p>.05$. 
Figure 4. Percentage of Children Who Improved, Worsened or Remained the Same Between Workshop Two and Workshop Three in the SCIT and TAU Groups - Parent Report $(\geq 15 \%$ change used as benchmark)

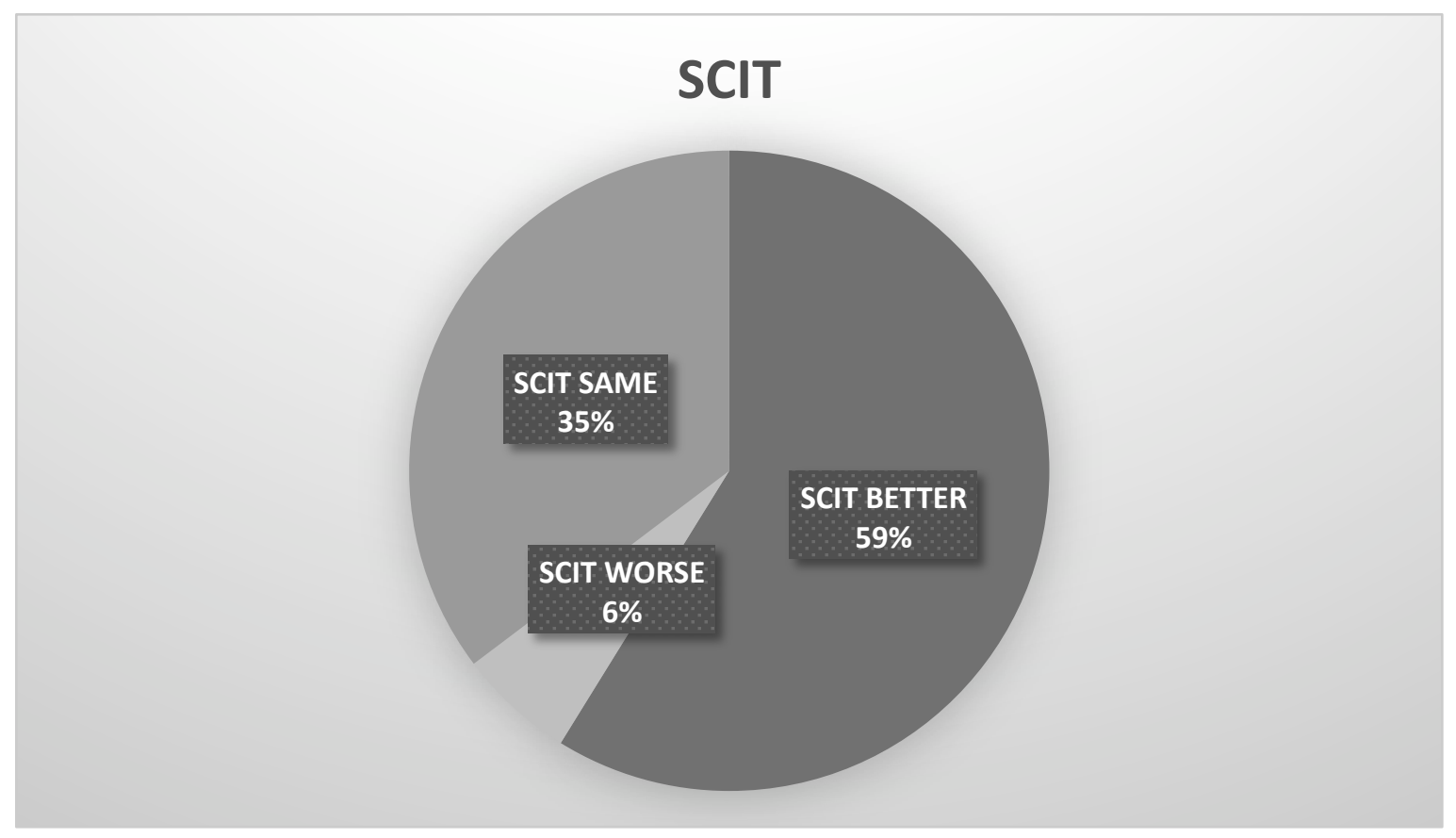

\section{TAU}

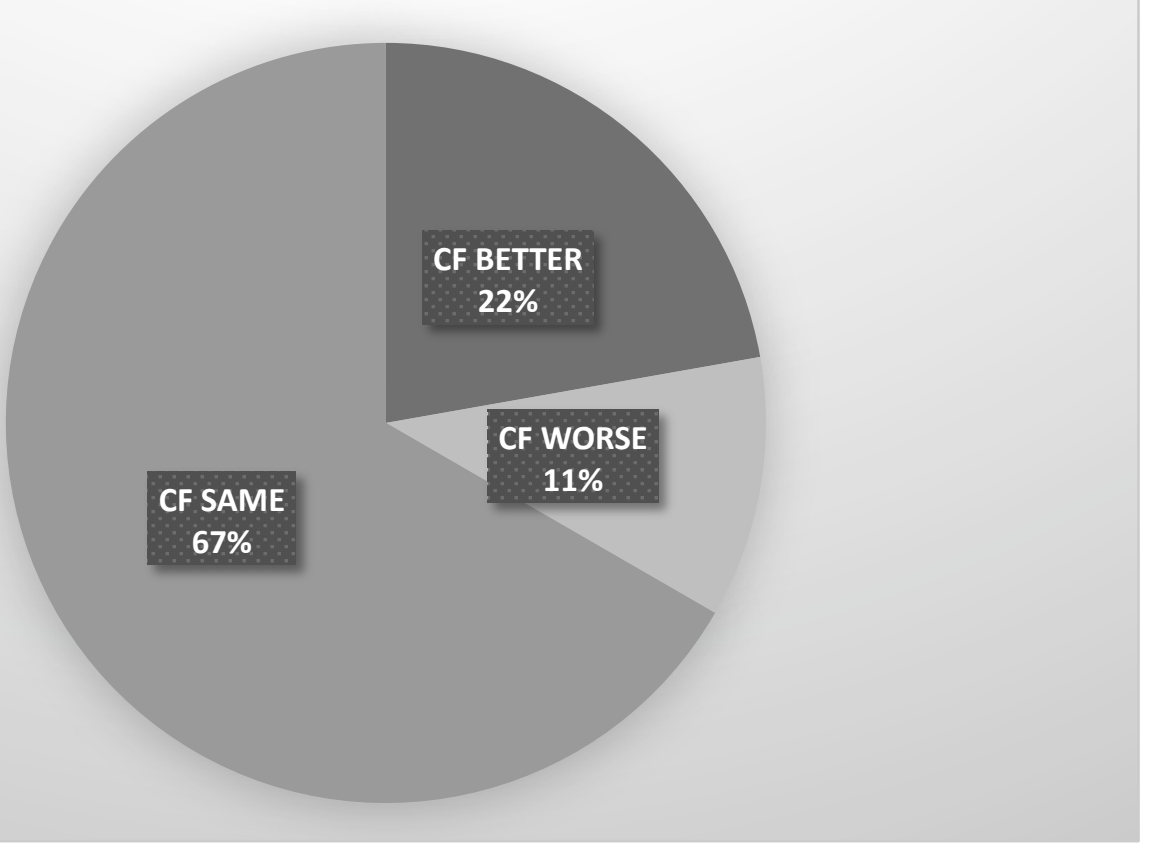


Figure 5. Therapist ECBI Change throughout the First, Second, and Third Workshops between the $\operatorname{SCIT}(N=16)$ and TAU $(N=21)$ Groups

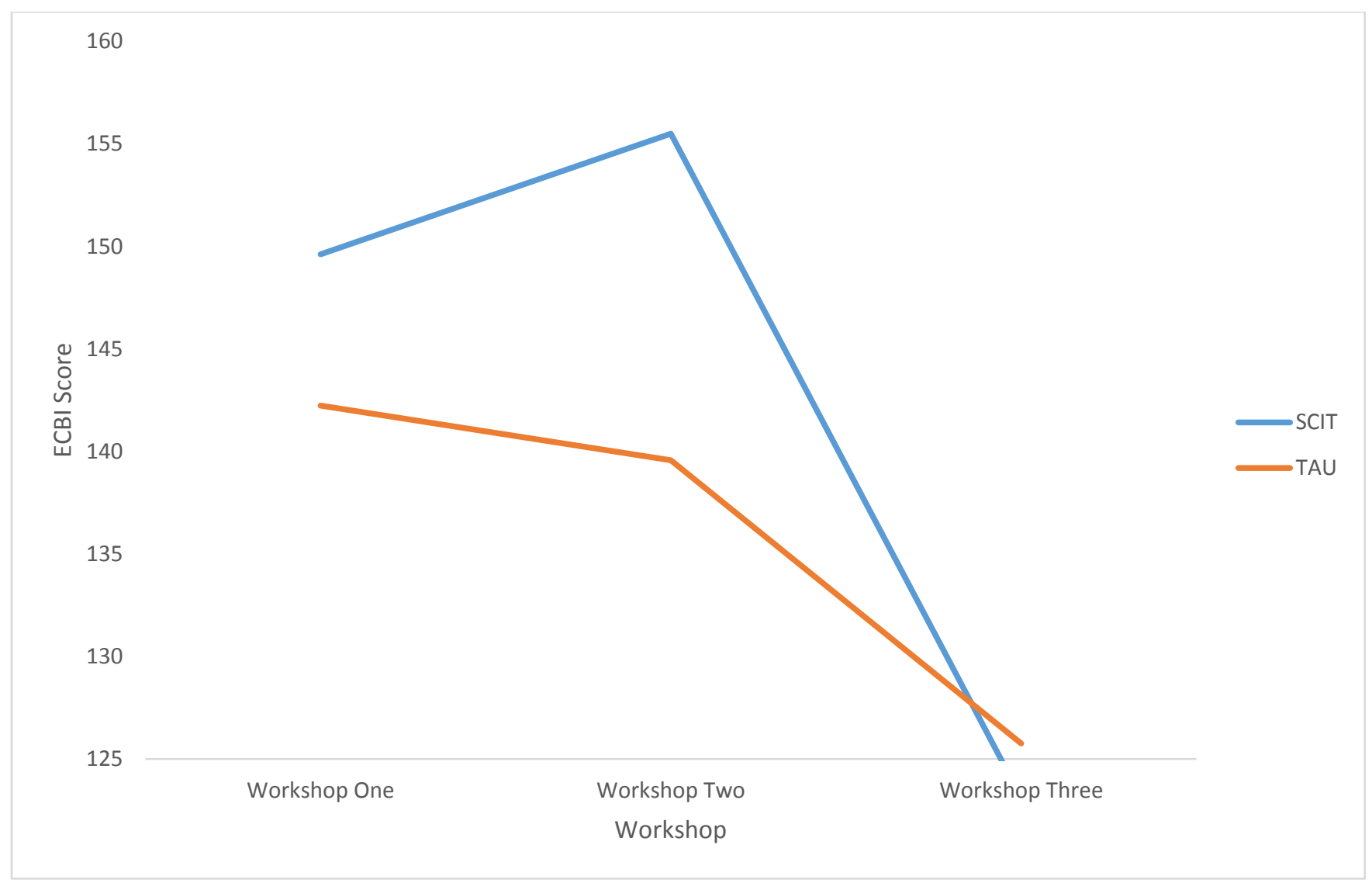


Figure 6. Therapist ECBI Change throughout the Second and Third Workshops between the $\operatorname{SCIT}(N=18)$ and TAU $(N=21)$ Groups

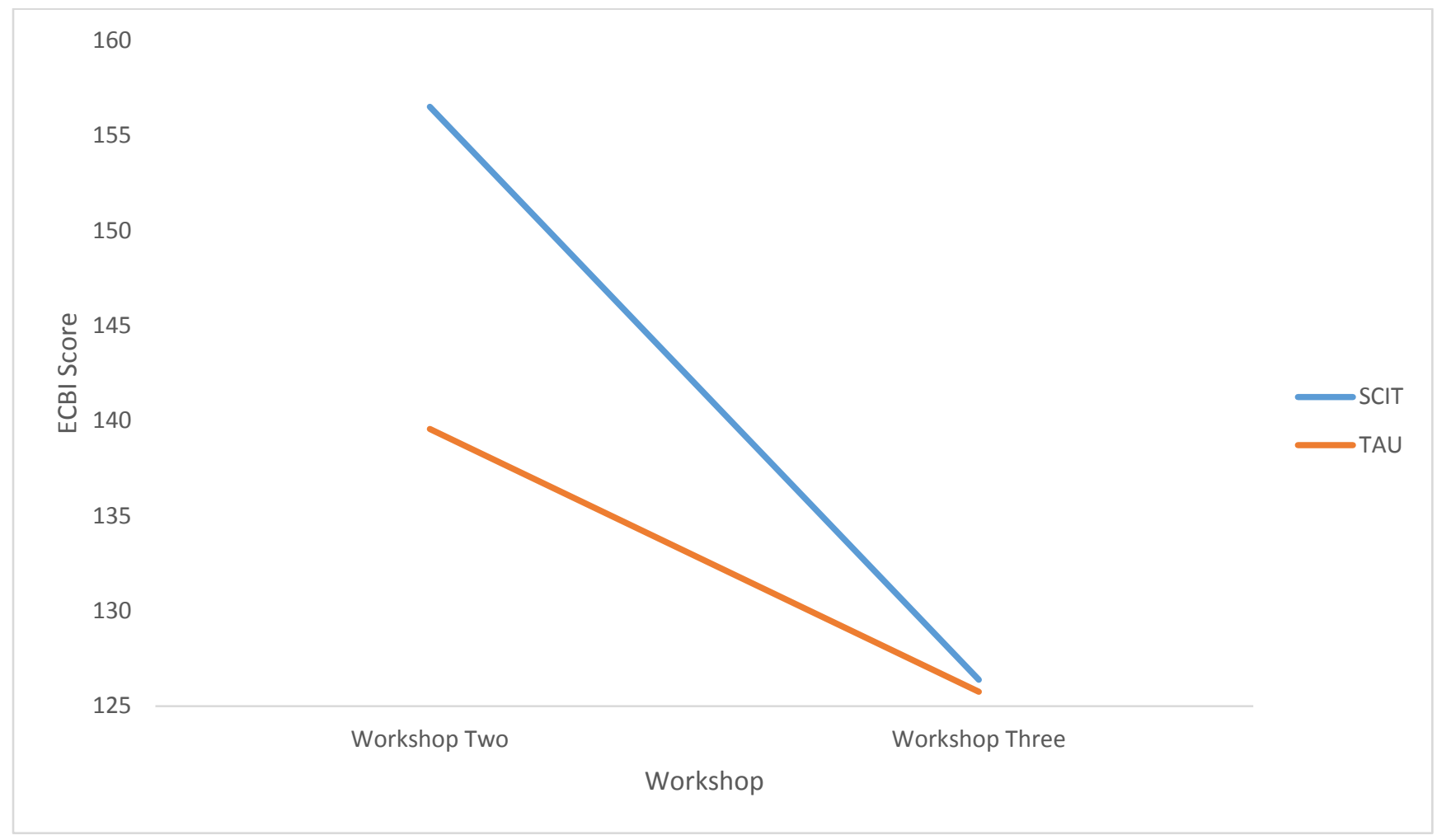


Figure 7. Percentage of Children Who Improved, Worsened or Remained the Same Between Workshop One and Workshop Three in the SCIT and TAU Groups - Therapist Report $(\geq 15 \%$ change used as benchmark).

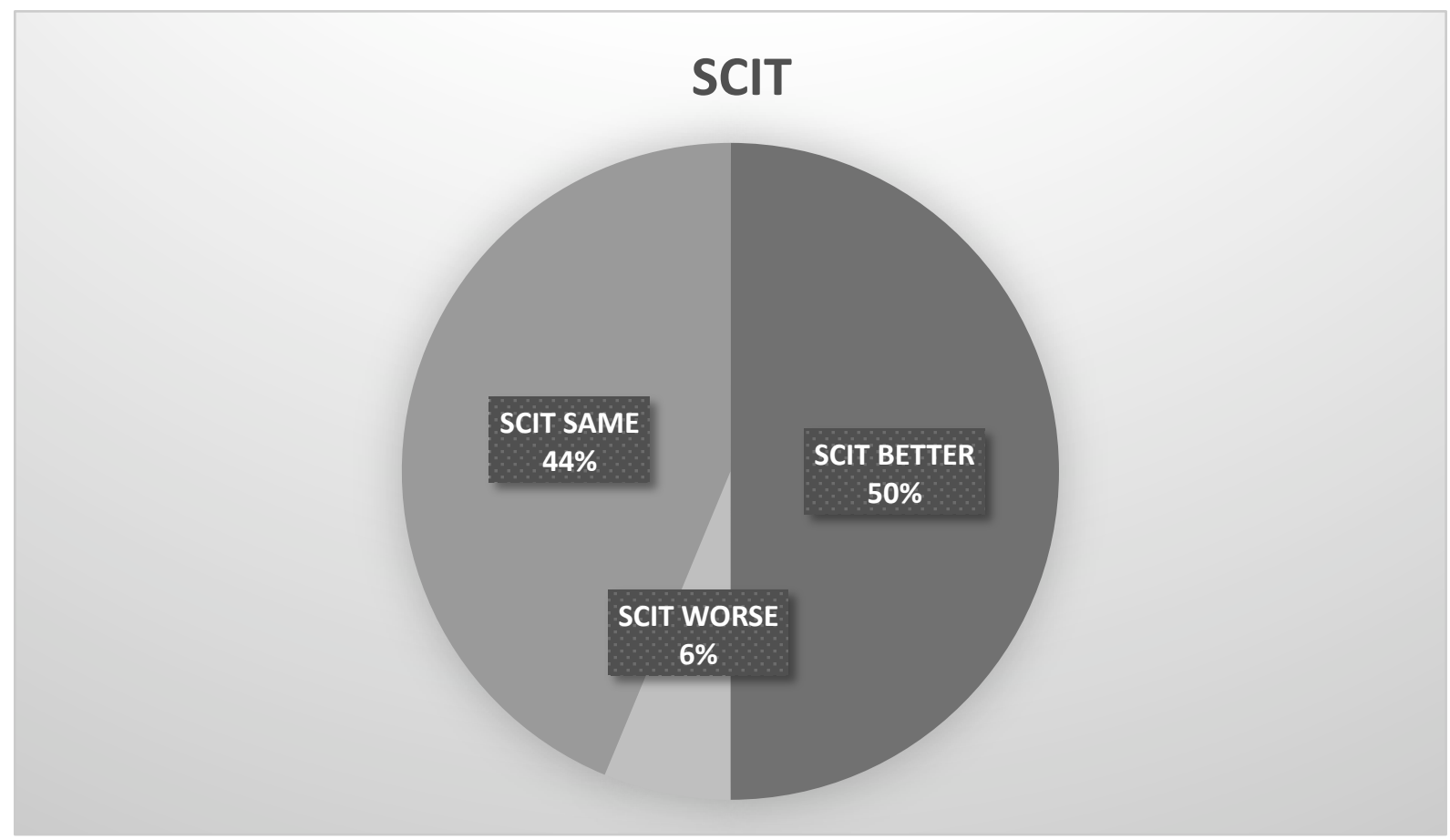

\section{TAU}

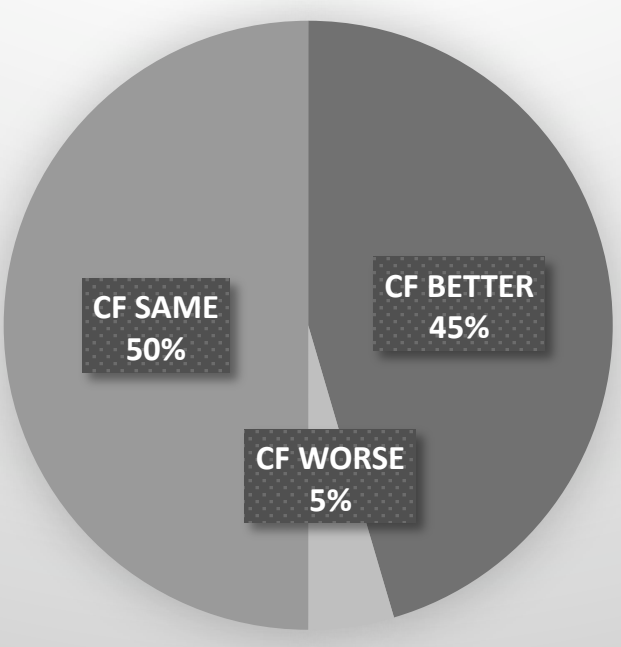


Figure 8. Percentage of Children Who Improved, Worsened or Remained the Same Between Workshop Two and Workshop Three in the SCIT and TAU Groups - Therapist Report $(\geq 15 \%$ change used as benchmark)

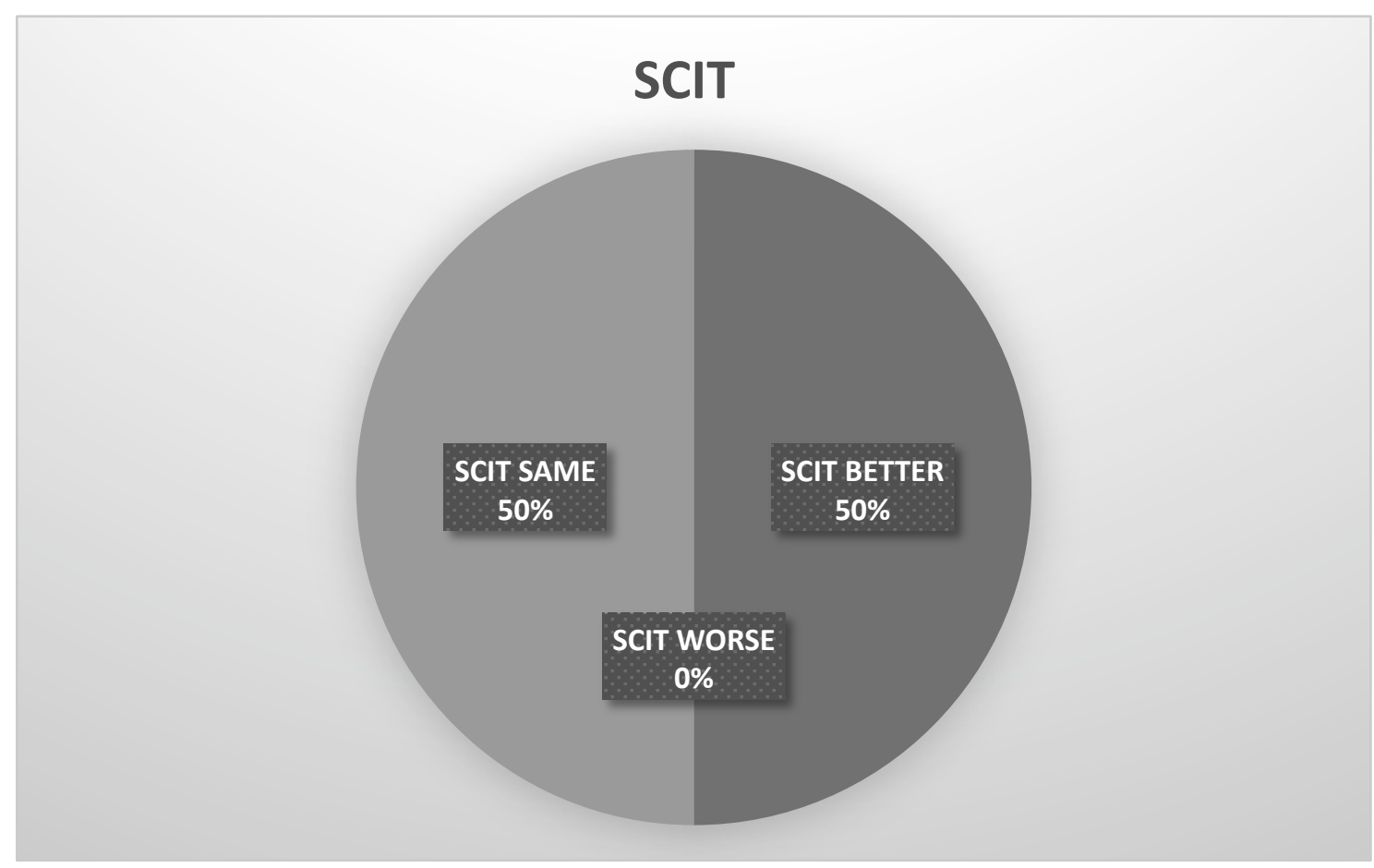

\section{TAU}

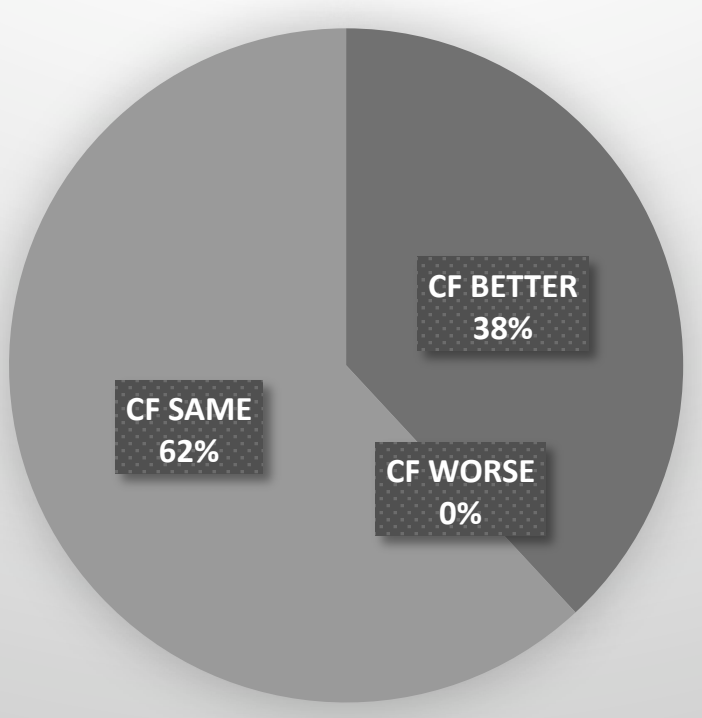


Figure 9. Staff Sense of Competence across Workshops.

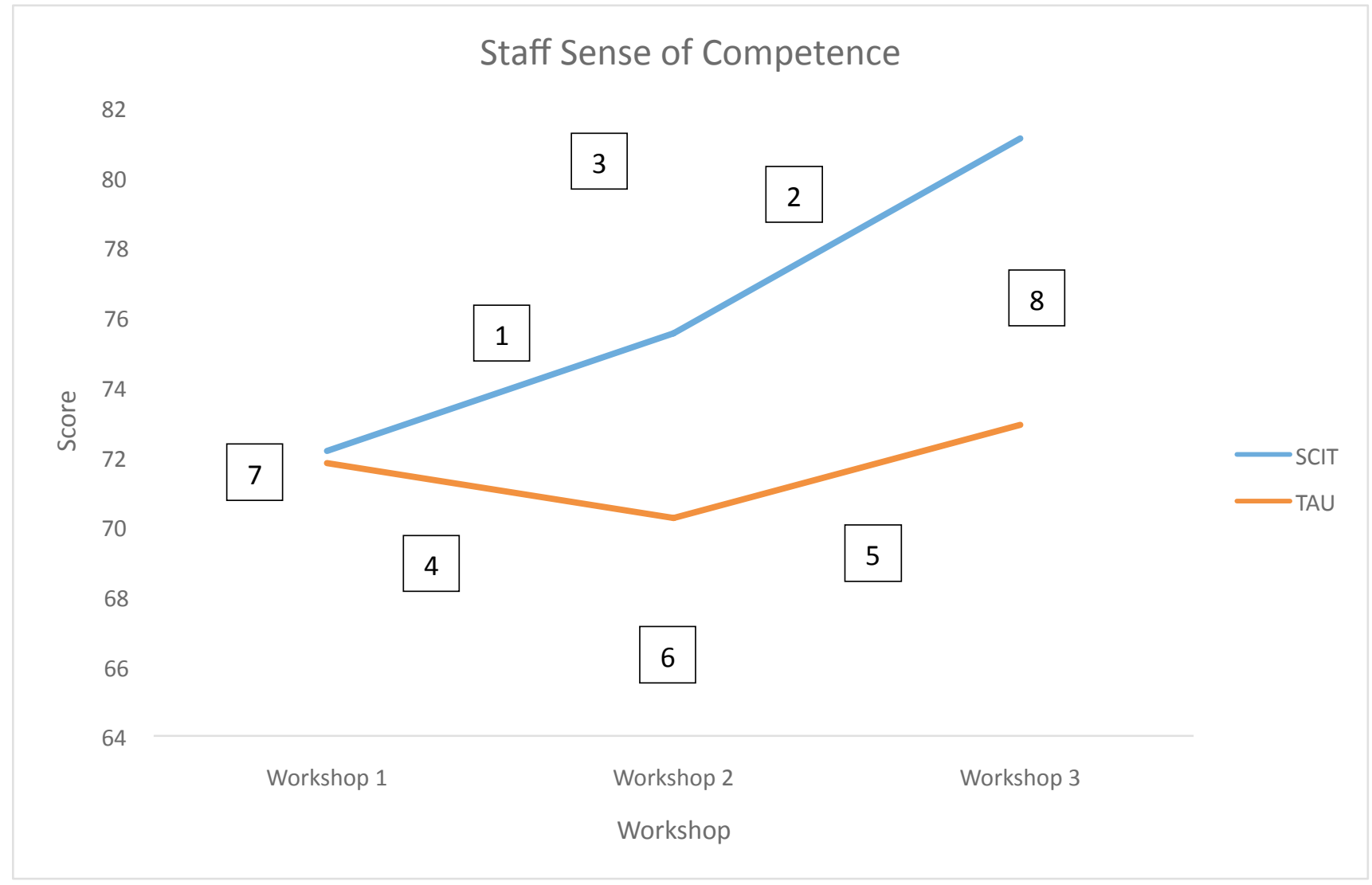

Note:

1. Post hoc comparisons using the Tukey HSD test indicated that the mean score for the SCIT group at workshop one $(M=72.16 .79, S D$

$=10.42)$ was not significantly different from the mean score for the SCIT group at workshop two $(M=75.52, S D=9.82), p>.05$.

2. Post hoc comparisons using the Tukey HSD test indicated that the mean score for the SCIT group at workshop two $(M=75.52, S D=$ 9.82) was significantly different from the mean score for the SCIT group at workshop three $(M=81.11, S D=11.03), p<.05$.

3. Post hoc comparisons using the Tukey HSD test indicated that the mean score for the SCIT group at workshop one $(M=72.16 .79, S D$

$=10.42)$ was significantly different from the mean score for the SCIT group at workshop three $(M=81.11, S D=11.03), p<.01$.

4. Post hoc comparisons using the Tukey HSD test indicated that the mean score for the TAU group at workshop one $(M=71.81, S D=$ 9.91) was not significantly different from the mean score for the TAU group at workshop two $(M=70.92, S D=7.29), p>.05$.

5. Post hoc comparisons using the Tukey HSD test indicated that the mean score for the TAU group at workshop two $(M=70.92, S D=$ 7.29) was not significantly different from the mean score for the TAU group at workshop three $(M=72.90, S D=9.55), p>.05$.

6. Post hoc comparisons using the Tukey HSD test indicated that the mean score for the TAU group at workshop one $(M=71.81, S D=$ 9.91) was not significantly different from the mean score for the TAU group at workshop three $(M=72.90, S D=9.55), p>.05$.

7. Post hoc comparisons using the Tukey HSD test indicated that the mean score for the SCIT group at workshop one $(M=72.16 .79, S D$

$=10.42)$ was not significantly different from the mean score for the TAU group at workshop one $(M=71.81, S D=9.91), p>.05$.

8. Post hoc comparisons using the Tukey HSD test indicated that the mean score for the SCIT group at workshop three $(M=81.11, S D=$ 11.03), was significantly different from the mean score for the TAU group at workshop three $(M=72.90, S D=9.55), p<.01$. 
Appendix A: Outline of a Typical SCIT Session

\begin{tabular}{|c|c|}
\hline Time & Procedures \\
\hline $1 \mathrm{~min}$. & 1. Prep for session \\
\hline 5 min. & 2. Check-in with parent; review CDI homework \\
\hline $3 \mathrm{~min}$. & 3. Model CDI skills with child \\
\hline 2 min. & 4. Discuss model with parent \\
\hline $5 \mathrm{~min}$. & 5. Have parent practice CDI skills with child \\
\hline $2 \mathrm{~min}$. & 6. Give feedback to parent \\
\hline $5 \mathrm{~min}$. & 7. Have parent practice CDI skills with child \\
\hline $2 \mathrm{~min}$. & 8. Give feedback to parent \\
\hline & 9. Break \\
\hline $2 \mathrm{~min}$. & 10. Review ADI homework \\
\hline $2 \mathrm{~min}$. & 11. Have parent select 3 possible privileges to restrict \\
\hline $3 \mathrm{~min}$. & 12. Model ADI skills with child \\
\hline $2 \mathrm{~min}$. & 13. Discuss model with parent \\
\hline $5 \mathrm{~min}$. & 14. Have parent practice ADI skills with child \\
\hline $2 \mathrm{~min}$. & 15. Give feedback to parent \\
\hline 5 min. & 16. Have parent practice ADI skills with child \\
\hline $2 \mathrm{~min}$. & 17. Give feedback to parent \\
\hline $1 \mathrm{~min}$. & 18. Assign CDI and ADI homework (Pg. 69 \& 70) \\
\hline $1 \mathrm{~min}$. & 19. Complete fidelity checklist \\
\hline $50 \mathrm{~min}$ & TOTAL TIME \\
\hline
\end{tabular}


Appendix B: Requirements for Certification- PCIT versus SCIT (Parent-Child Interaction Therapy, 2016)

\begin{tabular}{|l|l|}
\hline \multicolumn{1}{|c|}{ Parent-Child Interaction Therapy (PCIT) } & \multicolumn{1}{|c|}{ Staff-Child Interaction Therapy (SCIT) } \\
\hline $\begin{array}{l}\text { 5 day PCIT workshop and 2 day advanced } \\
\text { training workshop by Master Trainer }\end{array}$ & 3 day workshop (two full day, 1 half day) \\
\hline $\begin{array}{l}1 \text { year/2x monthly consultation by Master } \\
\text { Trainer }\end{array}$ & $\begin{array}{l}3 \text { consultation calls with a graduate student } \\
\text { SCIT trainer between } 2^{\text {nd }} \text { and } 3^{\text {rd }} \text { workshop }\end{array}$ \\
\hline $\begin{array}{l}\text { Review and approval of video tapes by a } \\
\text { PCIT master trainer }\end{array}$ & Submission of SCIT manual to SCIT therapist \\
\hline $\begin{array}{l}\text { Completion of two full PCIT cases to } \\
\text { graduation }\end{array}$ & Completion of 1 SCIT case \\
\hline $\begin{array}{l}\text { Master's degree in mental health field/ under } \\
\text { supervision by licensed provider }\end{array}$ & Bachelors or Master's degree \\
\hline Outpatient clinic setting & Home-based service \\
\hline Child clients 2-7 years old & Child clients 2-9 years old \\
\hline
\end{tabular}


Appendix C: Differences between PCIT and SCIT (McNeil \& Hembree-Kigin, 2010)

\begin{tabular}{|c|c|}
\hline Parent Child Interaction Therapy (PCIT) & Staff Child Interaction Therapy (SCIT) \\
\hline General Format & General Format \\
\hline $\begin{array}{l}\text { 1. Delivered in an outpatient clinic } \\
\text { setting. The therapist is positioned in } \\
\text { the therapy room with the caregiver } \\
\text { and child or behind a one-way mirror } \\
\text { and speaking to the parent through a } \\
\text { cell phone or bug-in the ear blue tooth } \\
\text { device. }\end{array}$ & $\begin{array}{l}\text { 1. Delivered in the child client's home } \\
\text { setting. The therapist, caregiver and } \\
\text { child are seated at a table or on the } \\
\text { floor in a room with a door on a small } \\
\text { blanket or carpet. }\end{array}$ \\
\hline $\begin{array}{l}\text { 2. Treatment sessions occur for } 1 \text { hour, } \\
\text { once per week. }\end{array}$ & $\begin{array}{l}\text { 2. Treatment sessions occur for } 1 \text { hour } \\
\text { each time the therapist enters the } \\
\text { home (may be more than once/week). }\end{array}$ \\
\hline $\begin{array}{l}\text { 3. Caregiver behavior modification is } \\
\text { done through therapist coaching. }\end{array}$ & $\begin{array}{l}\text { 3. Caregiver behavior modification is } \\
\text { done using modeling, coding, and } \\
\text { feedback. }\end{array}$ \\
\hline $\begin{array}{l}\text { 4. Caregivers work toward mastery of } \\
\text { CDI exclusively. Once CDI mastery is } \\
\text { met, caregivers progress toward } \\
\text { mastery of PDI. }\end{array}$ & $\begin{array}{l}\text { 4. Caregivers will simultaneously work } \\
\text { toward mastery of both CDI and ADI } \\
\text { during each session. }\end{array}$ \\
\hline $\begin{array}{l}\text { 5. Treatment will conclude when the } \\
\text { child client's score on the Eyberg } \\
\text { Child Behavior Inventory (ECBI) falls } \\
\text { within normal limits, the parent has } \\
\text { reached mastery on CDI and PDI } \\
\text { skills and the parent feels confident in } \\
\text { his or her ability to manage the child's } \\
\text { behavior. }\end{array}$ & $\begin{array}{l}\text { 5. Treatment will conclude following } 18 \\
\text { sessions or a predetermined 6-8 week } \\
\text { interval (whichever occurs first) } \\
\text { regardless of parental mastery or skills } \\
\text { acquisition. }\end{array}$ \\
\hline $\begin{array}{l}\text { 6. An Eyberg Behavior Inventory is } \\
\text { completed at the beginning of each } \\
\text { session. }\end{array}$ & $\begin{array}{l}\text { 6. An Eyberg Behavior Inventory is } \\
\text { completed only at pre-treatment and } \\
\text { post-treatment. }\end{array}$ \\
\hline Child-Directed Interaction & Child-Directed Interaction \\
\hline $\begin{array}{l}\text { 7. If a child becomes aggressive toward } \\
\text { the parent or destructive toward an } \\
\text { object, the respective parent or object } \\
\text { may be briefly removed from the } \\
\text { room to prevent further escalation. }\end{array}$ & $\begin{array}{l}\text { 6. If a child becomes aggressive toward } \\
\text { the parent or destructive toward an } \\
\text { object, the parent will remain in the } \\
\text { same room but will turn with his or } \\
\text { her back toward the child for } \\
\text { approximately } 30 \text { seconds or until the } \\
\text { child is calm. The therapist will } \\
\text { supervise the child. Once the child is } \\
\text { calm, the parent will re-engage the } \\
\text { child in play. }\end{array}$ \\
\hline Parent-Directed Interaction & Changed to Adult-Directed Interaction \\
\hline 7. A time-out sequence is utilized upon & 7. A broken record technique and \\
\hline
\end{tabular}




\begin{tabular}{|l|l|}
\hline $\begin{array}{l}\text { noncompliance to parental command } \\
\text { or violation of predetermined house } \\
\text { rule. }\end{array}$ & $\begin{array}{l}\text { restriction of privilege procedure is } \\
\text { utilized upon noncompliance to } \\
\text { parental command. No physical } \\
\text { movement removal of the child is } \\
\text { conducted. }\end{array}$ \\
\hline $\begin{array}{l}\text { 8. PDI homework practice is } \\
\text { progressively generalized outside of } \\
\text { play commands to real life commands } \\
\text { and into the public environment. }\end{array}$ & $\begin{array}{l}\text { ADI homework practice does not } \\
\text { generalize beyond effective } \\
\text { commands in play situations. }\end{array}$ \\
\hline
\end{tabular}

Similarities between PCIT (McNeil \& Hembree-Kigin, 2010) and SCIT are detailed in the table below:

\begin{tabular}{|c|}
\hline $\begin{array}{c}\text { Parent Child Interaction Therapy (PCIT) and Staff Child } \\
\text { Interaction Therapy (SCIT) }\end{array}$ \\
\hline General Format \\
\hline $\begin{array}{l}\text { 1. Caregivers, therapists, and child client's each participate } \\
\text { in session. } \\
\text { 2. Open-ended, non-rule directed toys are used during in- } \\
\text { session practice and homework. }\end{array}$ \\
\hline Child-Directed Interaction \\
\hline 3. Caregivers are assigned daily CDI homework practice. \\
\hline $\begin{array}{l}\text { 4. To reach mastery, caregivers must provide } 10 \text { labeled } \\
\text { praises, } 10 \text { behavior descriptions, } 10 \text { reflections and less } \\
\text { than } 3 \text { questions commands or negative talk in a } 5 \text { minute } \\
\text { coded interval. }\end{array}$ \\
\hline $\begin{array}{l}\text { 5. Selective attention is used to minimize minor } \\
\text { misbehavior. }\end{array}$ \\
\hline 6. The child leads the play. \\
\hline Adult-Directed Interaction \\
\hline $\begin{array}{l}\text { 7. Caregivers learn to deliver effective commands and } \\
\text { follow a pre-determined, step-by-step discipline } \\
\text { procedure. }\end{array}$ \\
\hline 8. Caregivers are assigned daily ADI homework practice. \\
\hline $\begin{array}{l}\text { 9. To reach mastery, caregivers must provide at least } 4 \\
\text { commands, at least } 75 \% \text { must be effective and correct } \\
\text { follow through to at least } 75 \% \text { of effective commands. }\end{array}$ \\
\hline $\begin{array}{l}\text { 10. Selective attention is used to minimize minor } \\
\text { misbehavior. }\end{array}$ \\
\hline 11. The parent leads the play. \\
\hline
\end{tabular}


Appendix D: Demographic Questionnaires (Stokes, 2014)

\section{Client Demographic Form}

How old is your child?

What is your child's sex?

Male Female

What mental disorder(s) has your child been diagnosed with? Please check all that apply.

$\square$ Autism Spectrum Disorder

$\square$ Attention Deficit Hyperactivity Disorder

$\square$ Oppositional Defiant Disorder

$\square$ Conduct Disorder

$\square$ Disruptive Behavior Disorder Not Otherwise Specified

$\square$ Post-Traumatic Stress Disorder

$\square$ Intellectual Disability

$\square$ Other Diagnosis (Please list below.)

How long has your child received mental health services?

Approximately how many hours of mental health services has your child received?

Have you been involved with Child Protective Services/ Child and Youth Services in the past?

Yes No

Are you currently involved with Child Protective Services/ Child and Youth Services?

Yes $\quad$ No 


\section{Therapeutic Support Staff Demographic Form}

(Stokes, 2014)

What is your sex?

Male

Female

What is your race/ ethnicity?

Caucasian

African American

Latino/ Hispanic

American Indian

Other: (Please list.)

Years in current position:

Highest degree earned: Some college

Associates degree

Bachelor's degree

Some graduate training

M. A. / M. S.

M. S. W. / L. S. W

Other: (Please list)

Are you a BCBA (Board certified behavior analyst)?

Yes

No

Are you a BCaBA (Board Certified assistant Behavior Analyst)?

Yes

No

Are you a RBT (Registered Behavior Technician)? 
Yes No

Have you ever received training that included coding of skill use and feedback based on coding of skill use in the past?

Yes $\quad$ No

Have you ever heard of Parent-Child Interaction Therapy?

Yes $\quad$ No

Have you received training in Parent-Child Interaction Therapy in the past?

Yes $\quad$ No

Have you ever observed a Parent-Child Interaction Therapy session?

Yes $\quad$ No

Please describe all training you have received at Family Behavioral Resources or elsewhere in the form of a workshop and list the length of the training (please consider workshops on therapeutic techniques and behavior modification).

Workshop Title Length

Please describe all training you have received at Family Behavioral Resources or elsewhere in the form of a class and list the length of the training (please consider classes on therapeutic techniques and behavior modification)

Workshop Title Length 
How long have you worked with your client?

How many hours do you typically spend with your client each week?

Has your client been involved with Child Protective Services/ Child and Youth Services in the past?

Yes $\quad$ No

Is your client currently involved with Child Protective Services/ Child and Youth Services?

Yes No

What mental disorder(s) has your client been diagnosed with? Please check all that apply.

$\square$ Autism Spectrum Disorder

$\square$ Attention Deficit Hyperactivity Disorder

$\square$ Oppositional Defiant Disorder

$\square$ Conduct Disorder

$\square$ Disruptive Behavior Disorder Not Otherwise Specified

$\square$ Post-Traumatic Stress Disorder

$\square$ Intellectual Disability

$\square$ Other Diagnosis (Please list below.) 
Appendix E: TSS Workshop Satisfaction Survey (Stokes, 2014)

Therapist ID \#

DATE

TSS Workshop Satisfaction Survey

1) Please rate this workshop in terms of meeting your needs or expectations.

Excellent

Satisfactory

Neutral

Unsatisfactory

Poor

2) The workshop facilities were comfortable and appropriate.

Strongly Disagree

Disagree

Undecided

Agree

Strongly Agree

3) The instructors were knowledgeable in the subject area.

Strongly Disagree

Disagree

Neutral

Agree

Strongly Agree

4) The assignments and activities were appropriate.

Strongly Disagree

Disagree

Neutral

Agree

Strongly Agree 
Appendix F: SCIT Final Survey

\section{SCIT Final Survey}

1. What changes (if any) have you noticed with your client since beginning SCIT?

2. What are some things that could make SCIT better?

3. How do you feel SCIT has affected how your client's caregiver interacts with your client?

4. What were you hoping to gain from implementing SCIT with your client?

5. What was the biggest challenge you faced in implementing SCIT? 


\section{Appendix G: Staff Sense of Competence Scale}

\section{Staff Sense of Competence Scale}

Based on Parent Sense of Competence Scale (Gibaud-Wallston \& Wandersman, 1978 in Johnston \& Mash, 1989)

Please rate the extent to which you agree or disagree with each of the following statements.

$\begin{array}{cccccc}\begin{array}{c}\text { Strongly } \\ \text { Disagree }\end{array} & \begin{array}{c}\text { Somewhat } \\ \text { Disagree }\end{array} & \text { Disagree } & \text { Agree } & \begin{array}{c}\text { Somewhat } \\ \text { Agree }\end{array} & \begin{array}{c}\text { Strongly } \\ \text { Agree }\end{array} \\ 1 & 2 & 3 & 4 & 5 & 6\end{array}$

1. The problems of working with a child are easy to solve once you know how your actions affect the child, an understanding I have acquired.

2. Even though being a TSS could be rewarding, I am frustrated now while my client is at his / her present age.

3. I go to bed the same way I wake up in the morning, feeling I have not accomplished a whole lot.

4. I do not know why it is, but sometimes when I'm supposed to be in control, I feel more like the one being manipulated by my client.

5. Other TSS are better prepared to be an effective than I am.

6. I would make a fine model for a new TSS to follow in order to learn what he/she would need to know in order to be an effective TSS.

7. Being a TSS is manageable, and any problems are easily solved.

8. A difficult problem in being a TSS is not knowing whether you're doing a good job or a bad one.

9. Sometimes I feel like I'm not getting anything done.

10. I meet by own personal expectations for expertise in working with my client $\begin{array}{llllll}1 & 2 & 3 & 4 & 5 & 6\end{array}$

11. If anyone can find the answer to what is troubling my client, I am the one $\quad \begin{array}{llllll}1 & 2 & 3 & 4 & 5 & 6\end{array}$

12. My talents and interests are in other areas, not being a TSS.

$\begin{array}{llllll}1 & 2 & 3 & 4 & 5 & 6\end{array}$

13. Considering how long I've been a TSS, I feel thoroughly familiar with this role.

14. If being a TSS of a child were only more interesting, I would be motivated to do a better job.

15. I honestly believe I have all the skills necessary to be an effective TSS with my client

16. Being a TSS makes me tense and anxious. 
Appendix H: Timeline of SCIT and Treatment as Usual Workshops

\begin{tabular}{|l|l|l|l|}
\hline Condition & $\begin{array}{l}\text { Day One SCIT } \\
\text { Workshop }\end{array}$ & $\begin{array}{l}\text { Day Two Intensive } \\
\text { Skills Workshop }\end{array}$ & $\begin{array}{l}\text { Post-Treatment } \\
\text { Evaluation and } \\
\text { Workshop }\end{array}$ \\
\hline SCIT & $\begin{array}{l}\text { Learn rationale for } \\
\text { SCIT. Begin to } \\
\text { practice CDI and ADI } \\
\text { skills. Allowed to } \\
\text { practice PRIDE skills } \\
\text { with client but do not } \\
\text { begin SCIT. }\end{array}$ & $\begin{array}{l}\text { Practice CDI and ADI } \\
\text { skills to mastery. } \\
\text { Practice } \\
\text { implementation of } \\
\text { skills within treatment } \\
\text { protocol. Begin SCIT } \\
\text { with client and } \\
\text { participate in } \\
\text { consultation calls } \\
\text { following workshop. }\end{array}$ & $\begin{array}{l}\text { Implementation of } \\
\text { SCIT for research } \\
\text { concludes. Focus of } \\
\text { workshop is on SCIT } \\
\text { reflection, question and } \\
\text { answer session. }\end{array}$ \\
\hline
\end{tabular}

\begin{tabular}{|c|c|c|c|}
\hline Condition & $\begin{array}{l}\text { TAU Day One } \\
\text { Workshop } \\
\text { (Compassion Fatigue) }\end{array}$ & $\begin{array}{l}\text { TAU Day Two } \\
\text { Workshop } \\
\text { (Compassion Fatigue) }\end{array}$ & $\begin{array}{l}\text { Post-Treatment } \\
\text { Evaluation and } \\
\text { Workshop }\end{array}$ \\
\hline $\begin{array}{l}\text { Treatment As } \\
\text { Usual (TAU) }\end{array}$ & $\begin{array}{l}\text { Learn definition of and } \\
\text { application of } \\
\text { compassion fatigue } \\
\text { (e.g., risk factors; } \\
\text { stress management). } \\
\text { Discuss behavioral } \\
\text { health rehabilitative } \\
\text { services (treatment as } \\
\text { usual). No SCIT skills } \\
\text { are provided. }\end{array}$ & $\begin{array}{l}\text { Discuss rationale for } \\
\text { discussion of working } \\
\text { with clients' exposed } \\
\text { to trauma as exposure } \\
\text { to prevent against } \\
\text { compassion fatigue. } \\
\text { Discuss and practice } \\
\text { additional forms of } \\
\text { prevention against } \\
\text { compassion fatigue } \\
\text { (e.g., meditation). No } \\
\text { SCIT skills are } \\
\text { provided. Treatment } \\
\text { sessions continue as } \\
\text { usual. }\end{array}$ & $\begin{array}{l}\text { TAU therapists receive } \\
\text { brief overview of SCIT } \\
\text { in powerpoint format. }\end{array}$ \\
\hline
\end{tabular}


Appendix I: SCIT and TAU Detailed Workshop Schedules

\section{SCIT Group Therapist Skill Workshop}

\begin{tabular}{|c|c|}
\hline $9: 00-9: 20$ & Arriving/Coffee/ Sign in Sheets/ Name Tags \\
\hline $9: 20-10: 00$ & Welcome Presentation/ Informed Consent \\
\hline $10: 00-10: 15$ & $1^{\text {st }}$ Behavior Assessment Group \\
\hline $10: 15-10: 30$ & $2^{\text {nd }}$ Behavior Assessment Group \\
\hline $10: 30-10: 45$ & $3^{\text {rd }}$ Behavior Assessment Group \\
\hline $10: 45-11: 45$ & CDI/ DPICS/ Selective Attention Powerpoint \\
\hline $11: 45-12: 00$ noon & Break \\
\hline $12: 00-12: 45$ & $\begin{array}{l}\text { CDI/ DPICS/ Selective Attention Break Out } \\
\text { Practice }\end{array}$ \\
\hline $12: 45-1: 45$ & Lunch Break \\
\hline $1: 45-2: 00$ & DPICS Quiz \\
\hline $2: 00-2: 45$ & Effective Commands and ADI Powerpoint \\
\hline 2:45- 4:00 & ADI Break Out Practice; Finish CDI Mastery \\
\hline 4:00- 4:30 & $\begin{array}{l}\text { Conceptualize Skill Practice in Relation to } \\
\text { larger SCIT Treatment }\end{array}$ \\
\hline 4:30-5:00 & Reflection/ Satisfaction and Feedback Survey \\
\hline
\end{tabular}




\section{Treatment As Usual Day One Overview}

\begin{tabular}{|l|l|}
\hline $9: 00-9: 45$ & $\begin{array}{r}\text { Sign in/Breakfast } \\
\text { Get to know you games } \\
\text { What we're doing today slide } \\
\text { Consent }\end{array}$ \\
\hline $9: 50-10: 50$ & Assessment/Measures \\
\hline $10: 50-11: 05$ & Finish Measures/Break \\
\hline $11: 05-12: 40$ & TAU Fidelity Break Out Group \\
\hline $12: 40-1: 40$ & Lunch Break \\
\hline $1: 40-2: 40$ & $\begin{array}{l}\text { Compassion Fatigue presentation/ end with } \\
\text { relaxation practice }\end{array}$ \\
\hline $2: 40-2: 55$ & Break \\
\hline $2: 55-3: 15$ & Movie \\
\hline $3: 15-3: 45$ & Social Support Discussion \\
\hline $3: 45-4: 05$ & Ball Toss Game \\
\hline $4: 05-4: 15$ & Assessment Survey; reflection \\
\hline
\end{tabular}


SCIT Group Parent Skill Workshop

\begin{tabular}{|l|l|}
\multicolumn{2}{|c|}{ SCIT Group Parent Skill Workshop } \\
\hline 9:00-9:20 & $\begin{array}{l}\text { Arriving/Coffee/ Sign in Sheets/ Name } \\
\text { Tags/Consultation Call Sign in Sheets }\end{array}$ \\
\hline 9:20- 10:00 & Welcome Presentation \\
\hline $10: 00-10: 15$ & $\mathbf{1}^{\text {st }}$ Behavior Assessment Group \\
\hline $10: 15-10: 30$ & $\mathbf{2}^{\text {nd }}$ Behavior Assessment Group \\
\hline $10: 30-10: 45$ & $\mathbf{3}^{\text {rd }}$ Behavior Assessment Group \\
\hline $10: 45-11: 45$ & CDI/ DPICS/ Selective Attention Powerpoint \\
\hline $11: 45-12: 00$ noon & Break \\
\hline $12: 00-12: 45$ & $\begin{array}{l}\text { CDI/ DPICS/ Selective Attention Break Out } \\
\text { Practice }\end{array}$ \\
\hline $12: 45-1: 45$ & Lunch Break \\
\hline $1: 45-2: 15$ & Effective Commands and ADI Powerpoint \\
\hline $2: 15-3: 15$ & ADI Break Out Practice \\
\hline $3: 15-4: 30$ & $\begin{array}{l}\text { Third SCIT Session Role Play; Finish CDI/ADI } \\
\text { Mastery }\end{array}$ \\
\hline $4: 30-5: 00$ & Reflection/ Satisfaction and Feedback Survey \\
\hline
\end{tabular}


TAU Group Day Two Workshop Outline

Treatment as Usual Day Two Workshop

\begin{tabular}{|l|l|}
\hline $9: 00-9: 45$ & $\begin{array}{c}\text { Sign in/Breakfast } \\
\text { Get to know you games } \\
\text { What we're doing today slide }\end{array}$ \\
\hline $9: 50-11: 00$ & Assessment/Measures \\
\hline $11: 00-11: 15$ & Break \\
\hline $11: 15-12: 15$ & $\begin{array}{l}\text { Review Compassion Fatigue; discuss thoughts } \\
\text { since last workshop }\end{array}$ \\
\hline $12: 15-1: 30$ & Lunch Break \\
\hline $1: 30-2: 30$ & $\begin{array}{l}\text { Exposure and vicarious trauma } \\
\text { conceptualization, application to wrap around } \\
\text { therapists and discussion }\end{array}$ \\
\hline $2: 30-2: 45$ & Break \\
\hline $2: 45-3: 45$ & $\begin{array}{l}\text { Vicarious Trauma prevention; } \\
\text { relaxation/meditation practice }\end{array}$ \\
\hline $3: 45-4: 00$ & Break \\
\hline $4: 00-4: 15$ & Assessment Survey; reflection \\
\hline
\end{tabular}


SCIT Group Day Three Workshop Outline

SCIT Post-Treatment Evaluation and Workshop

\begin{tabular}{|l|l|}
\hline $9: 00-9: 20$ & Arriving/Coffee/ Sign in Sheets/ Name Tags \\
\hline $9: 20-10: 30$ & Behavior Assessment Groups \\
\hline $10: 30-11: 30$ & $\begin{array}{l}\text { SCIT Final Survey/ Question and Answer/ } \\
\text { Reflection Discussion }\end{array}$ \\
\hline 11:30-12:00 & Reflection/ Satisfaction and Feedback Survey \\
\hline
\end{tabular}

TAU Group Day Three Workshop Outline

Treatment as Usual Post-treatment Evaluation and Workshop

\begin{tabular}{|l|l|}
\hline 9:00 - 9:20 & Arriving/Coffee/ Sign in Sheets/ Name Tags \\
\hline $9: 20-10: 30$ & Behavior Assessment Groups \\
\hline 10:30-11:30 & SCIT Overview Presentation \\
\hline 11:30-12:00 & Reflection/ Satisfaction and Feedback Survey \\
\hline
\end{tabular}




\section{Appendix J: Adult Directed Interaction Command and Consequence Procedure (Norman \& McNeil, 2015)}

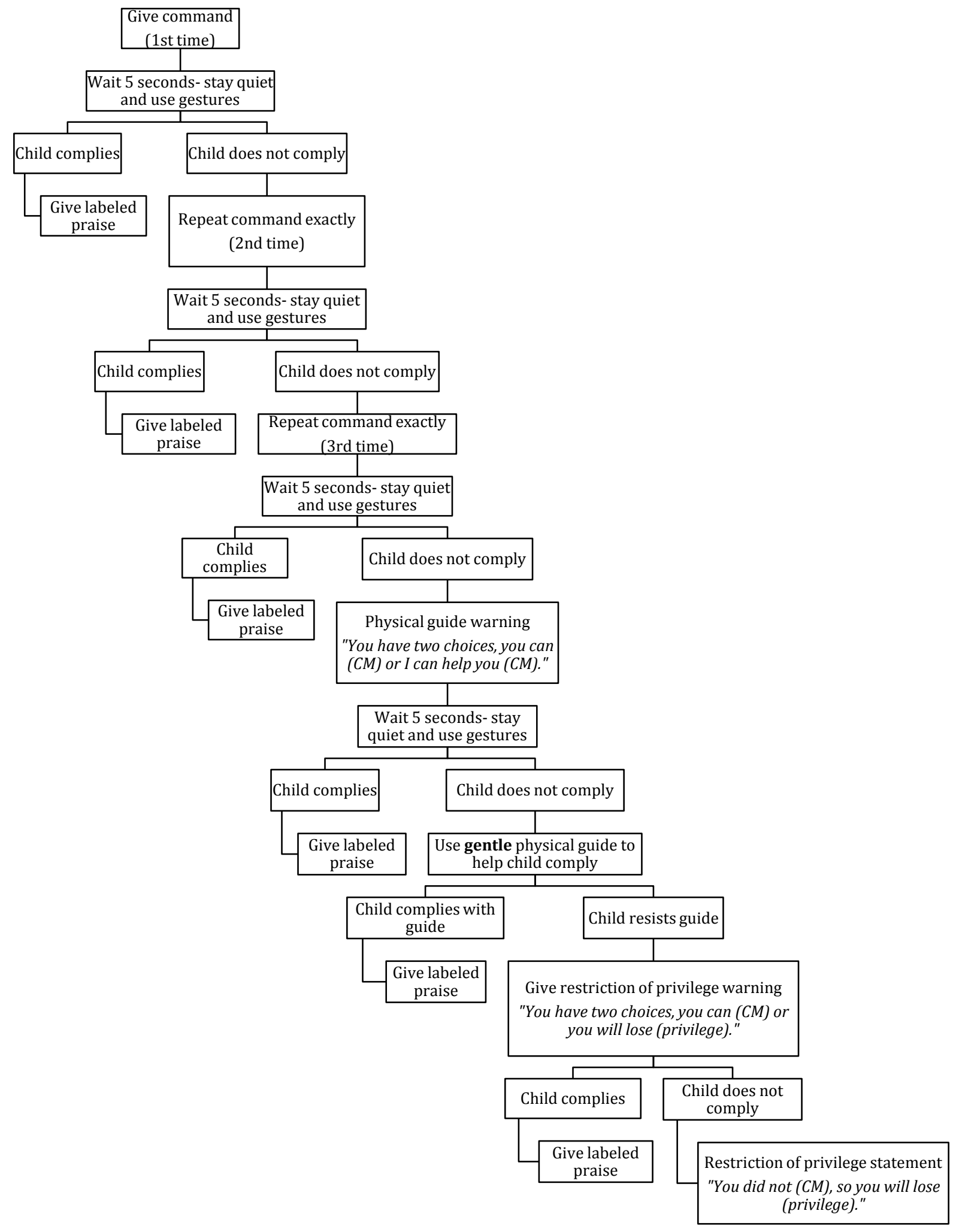


Appendix K: TAU Fidelity Discussion

TAU Fidelity Discussion

EMPHASIZE: HONESTY AND CONFIDENTIALITY OF RESPONSES

Day to day activities (conducting a session)

- On average, how long (Days? Hours?) do you spend with a consumer before they terminate?

- What are the top (3? 5?) treatment goals for your consumers?

- How do you measure consumer progress?

- What sort of data do you collect for a consumer? What does this data look like? How often do you collect this data?

- What does a typical session look like with this consumer?

- Walk us through: first 15 minutes, second 15 minutes?

- Can you describe, in detail, your last session with your consumer?

- What other variables could change how you conduct a session?

- Do you ever leave the home with the consumer and his/her family? How often? Where do you go? What do you do?

- How long do you usually see a consumer (total months/years).

- How would you describe your role in the life of your consumer?

- What determines when services conclude? How have cases concluded that you were a TSS for? How many cases have you had that you would say have "gotten better" and no longer needed help?

- What percentage of the session is spent working with the parent and child together? What percent is working with just the parent? Just the child?

Challenges in the home

- What are some common distractions in the home that keep you from completing your goals for a session? How often do these distractions arise?

- Describe the involvement the caregivers of your consumers have in a typical session (think back to your last session). How much time the caregiver was there (i.e. in the room- actively involved with what you were doing with the client)/How much time were you there? 
- What sorts of challenges arise when working with consumers?

- When challenges arise, what do you typically do about it?

○ If a child were to become very aggressive toward you or the parent, what do you do?

- If a child does not listen to you or the parent (in front of you) what do you do about it?

\section{Supervision}

- How often and for how long do you receive supervision?

- What percentage of the time that you're in the home of your consumer is your supervisor also in the home?

- What do you talk about in supervision? For how long? 
Appendix L: SCIT TSS Consultation Call Outline

Consultation Call \# 1

\section{SCIT TSS Consultation Call Outline}

SCIT Staff Member Name:

Date:

Time:

Text therapists the day before the call to remind - date/time/call in number. Tell them to have their manual at each call because they will be asked questions from it.

\section{BEGIN CALL}

(2 minutes) - Roster therapists

Therapist Name/ Length of time with client before SCIT workshop

1.

2.

3.

4.

5 .

6.

Ask all therapists how long they had worked with their client at the time that they attended the SCIT workshop (record above).

(10 to 30 minutes) - Check in

- Where therapists are at with their cases

- Answer questions during their check in (up to 5 min per therapist)

- Troubleshoot any client resistance or homework noncompliance

(1 min) - Remind about ECBI

- Have them tell you how many "yes" items were circled

Therapist Name

1 .

2.

3.

4.

5.

6.

(15 min) - Have them open manuals.

- Go over fidelity outline for first session. 
- Call on them to read certain bullets from the manual to make sure they are paying attention.

- Ask each therapist about paperwork. Coding. Progress sheets. Are they checking off fidelity?

- Have each therapist read progress sheet numbers to you. Have each therapist give homework data. Make them accountable and responsive.

(5-10 min) - Quiz therapists on DPICS - give sample verbalizations. Give them a 1-2-3 and have them say as a chorus how they would code it. Quiz them on ADI sequence.

(5-10 min)- Final questions. Reminders about next consult call. Reminder to bring completed manuals (*WITH 2 COMPLETED ECBI'S) to next workshop and time of next workshop.

\section{NOTES:}

** If a therapist has not started SCIT yet, have the sign up for a $4^{\text {th }}$ consultation call**

\section{Consultation Call \#}

\section{SCIT TSS Consultation Call Outline}

SCIT Staff Member Name:

Date:

Time:

Text therapists the day before the call to remind - date/time/call in number. Tell them to have their manual at each call because they will be asked questions from it.

\section{BEGIN CALL}

(2 minutes) - Roster therapists

Therapist Name/ Length of time with client before SCIT workshop

1.

2.

3.

4.

5 .

6.

(10 to 30 minutes) - Check in

- Where therapists are at with their cases

- Answer questions during their check in (up to 5 min per therapist) 
- Troubleshoot any client resistance or homework noncompliance

(1 min) - Remind about ECBI

- Have them tell you how many "yes" items were circled

Therapist Name

1.

2.

3.

4.

5.

6.

(15 min) - Have them open manuals.

- Go over fidelity outline for session.

- Call on them to read certain bullets from the manual to make sure they are paying attention.

- Ask each therapist about paperwork. Coding. Progress sheets. Are they checking off fidelity?

- Have each therapist read progress sheet numbers to you. Have each therapist give homework data. Make them accountable and responsive.

(5-10 min) - Quiz therapists on DPICS - give sample verbalizations. Give them a 1-2-3 and have them say as a chorus how they would code it. Quiz them on ADI sequence.

(5-10 min)- Final questions. Reminders about next consult call. Reminder to bring completed manuals (*WITH 2 COMPLETED ECBI'S) to next workshop and time of next workshop.

NOTES: 